\title{
GENERATION OF FINITE GROUPS OF LIE TYPE
}

BY

GARY M. SEITZ 1

\begin{abstract}
Let $p$ be an odd prime and $G$ a finite group of Lie type in characteristic other than $p$. Fix an elementary abelian $p$-subgroup of $\operatorname{Aut}(G)$. It is shown that in most cases $G$ is generated by the centralizers of the maximal subgroups of $E$. Results are established concerning the notions of layer generation and balance, and the strongly $p$-embedded subgroups of $\operatorname{Aut}(G)$ are determined.
\end{abstract}

1. Introduction. Let $p$ be an odd prime, $G$ a finite group, and $E \leqslant \operatorname{Aut}(G)$. Suppose that $E$ is an elementary abelian $p$-group and set $C_{G}^{0}(E)=\left\langle C_{G}(F): F\right.$ maximal in $E\rangle$. A very useful tool in the study of finite groups is the basic generation result: $C_{G}^{0}(E)=G$, provided the order of $G$ is relatively prime to $p$. The order restriction on $G$ is essential. For example, if $G=S_{2 p}$ is the symmetric group on $2 p$ letters and $E$ a Sylow $p$-subgroup of $G$, then $C_{G}^{0}(E)<G$. In this paper we consider the case of $G$ a finite group of Lie type and we show that generation usually holds. In addition, we prove results concerning the notions of layer generation, balance, and we determine the strongly $p$-embedded subgroups of $\operatorname{Aut}(G)$.

Let $G=G(q)$ be a finite simple group of Lie type defined over a field of characteristic $r$, and let $E \leqslant \operatorname{Aut}(G)=Y$ be an elementary abelian $p$-group with $r \neq p$, a prime. In order to state more useful results we consider multipliers and therefore introduce the following notation. Let $\tilde{G}$ denote an image of the universal covering group of $G$ (see [25] and (13.1), (13.3) of [23]) and let $\tilde{G} \unlhd \tilde{Y}$ be such that $\tilde{G} / Z(\tilde{G})=G$ and $\tilde{Y} / Z(\tilde{G})=Y$. Now let $\tilde{E}$ be a $p$-subgroup of $\tilde{Y}$ such that $\tilde{E} Z(\tilde{G}) / Z(\tilde{G})=E$. We will prove the following results.

THEOREM 1. Assume that $E$ is contained in the subgroup of $\operatorname{Aut}(G)$ generated by inner and diagonal automorphisms. Then either $C_{\tilde{G}}^{Q}(\tilde{E})=\tilde{G}$ or one of the following holds:

(i) $p=3$ and $G \cong P S p(n, 2)^{\prime}, P S U(n, 2)$, or $O^{\varepsilon}(n, 2)^{\prime}, \varepsilon=(-1)^{n / 2}$.

(ii) $p=3$ and $G \cong G_{2}(2)^{\prime}, F_{4}(2), E_{6}(2),{ }^{2} E_{6}(2), E_{7}(2)$, or $E_{8}(2)$.

(iii) $p=3$ and $G \cong P S L\left(3^{k}, 4\right),{ }^{2} F_{4}(2)^{\prime}, F_{4}(4), E_{6}(4)$ or $E_{8}(4)$.

(iv) $p=5$ and $G \cong{ }^{2} F_{4}(2)^{\prime}$.

Moreover, in each of the cases (i)-(iv) there exists a triple $(\tilde{G}, \tilde{E}, p)$ for which $C_{G}^{Q}(\tilde{E})<\tilde{G}$.

Received by the editors March 31, 1978 and, in revised form, September 2, 1980.

1980 Mathematics Subject Classification. Primary 20B25; Secondary 20D05.

'Supported in part by NSF Grant MCS 76-07015.

(C)1982 American Mathematical Society $0002-9947 / 81 / 0000-1069 / \$ 14.75$ 
THEOREM 2. Let $G_{1}$ be the subgroup of $\operatorname{Aut}(G)$ generated by inner and diagonal automorphisms of $G$. Assume that $E \$ G_{1}$. Then either $C_{G}^{\ell}(\tilde{E})=\tilde{G}$, or one of the following holds:

(i) If $e \in E-G_{1}$, then e induces a field automorphism on $G$ and the pair $\left(O^{r^{\prime}}\left(C_{G}(e)\right)\right.$, $E \cap G_{1}$ ) satisfies one of the conditions (i), (ii), or (iv) of Theorem 1.

(ii) $p=5$ and $G E \cong \operatorname{Aut}\left(\operatorname{Sz}\left(2^{5}\right)\right)$.

(iii) $p=3$ and $G E \cong \operatorname{Aut}\left(L_{2}(8)\right)$.

(iv) $p=3$ and $G E \cong D_{4}(2)\langle\sigma\rangle, D_{4}(4)\langle\sigma\rangle, O^{3^{\prime}}\left(\operatorname{Aut}\left(D_{4}(8)\right)\right),{ }^{3} D_{4}(2)\langle\sigma\rangle$, where in each case $\sigma$ is a graph automorphism of order 3 .

In each of the cases (i)-(iv), there exists a triple $(G, \tilde{E}, p)$ for which $C_{G}^{\ell}(\tilde{E})<\tilde{G}$.

An immediate corollary to Theorems 1 and 2 is the following.

THEOREM 3. If $p \geqslant 7$ or if $r>2$, then $C_{G}^{0}(E)=G$.

The above results can be used to obtain results on "layer generation" and "balance". With the above notation let $C_{\tilde{G}}^{r}(\tilde{E})=\left\langle O^{r^{\prime}}\left(C_{\tilde{G}}(F)\right): F\right.$ maximal in $\left.\tilde{E}\right\rangle$. As we will see in $\S 2, O^{r^{\prime}}\left(C_{\tilde{G}}(F)\right)=E\left(C_{\tilde{G}}(F)\right)$, if $q \geqslant 4$.

THEOREM 4 (LAYER GENERATION). If $E \cong Z_{p} \times Z_{p}$, then one of the following holds:

(i) $C_{\tilde{G}}^{Q}(\tilde{E})<\tilde{G}$.

(ii) $C_{\tilde{G}}^{r}(\tilde{E})=\tilde{G}$.

(iii) $p \mid q-1, G \cong L_{p}(q), E \leqslant G_{1}$ (as in Theorem 2 ), and $\tilde{E}$ is nonabelian.

(iv) $p \mid q+1, G \cong U_{p}(q), E \leqslant G_{1}$, and $\tilde{E}$ is nonabelian.

THeOREM 5 (BALANCE). If $E \cong Z_{p} \times Z_{p}$, then $\bigcap_{e \in E^{*}} O_{p^{\prime}}\left(C_{G}(e)\right)=1$ and one of the following holds:

(i) $\cap_{e \in E^{\sharp}} O_{p^{\prime}}\left(C_{X}(e)\right)=1$, whenever $G \leqslant X \leqslant \operatorname{Aut}(G)$.

(ii) $p \mid q-1, G \cong L_{p}(q), E \leqslant G_{1}$ (as in Theorem 2 ), and $\tilde{E}$ is nonabelian.

(iii) $p \mid q+1, G \cong U_{p}(q), E \leqslant G_{1}$, and $\tilde{E}$ is nonabelian.

THEOREM 6. Assume $p \geqslant 7$ and $(p,|\bar{W}|)=1$, where $\bar{W}$ is the Weyl group of the associated algebraic group of $G$. Then $C_{\tilde{G}}^{r}(\tilde{E})=\tilde{G}$.

THEOREM 7. Let $p$ be an arbitrary prime and assume that $X \leqslant \operatorname{Aut}(G)$ is a proper strongly p-embedded subgroup of $G X$. If $m_{p}(X) \geqslant 2$, then $X$ normalizes a Sylow p-subgroup of $G$ and one of the following holds:

(i) $p=3$ and $G \cong L_{3}(4)$;

(ii) $p=5$ and $G \cong S z\left(2^{5}\right)$ or ${ }^{2} F_{4}(2)^{\prime}$; or

(iii) $G \cong L_{2}(q), U_{3}(q), S z(q)$, or ${ }^{2} G_{2}(q)^{\prime}$, and $q$ is a power of $p$.

Much of the paper is concerned with the proof of Theorem 1, which is carried out in $\S \S 4$ and 5. Theorem 2 is proved in $\S 6$ and the remaining results are proved in $\S 7$. A considerable amount of work is involved in describing examples where generation fails to hold. This contributes substantially to the length of the paper.

In many cases it is easy to prove that generation holds. For example, if $\tilde{E}$ normalizes a proper $r$-subgroup of $\tilde{G}$, then $\tilde{G}=C_{\tilde{G}}^{r}(\tilde{E})=C_{\tilde{G}}^{\mathbf{0}}(\tilde{E})$ (see (2.3)). Also when $m_{p}(E) \geqslant 3$ and $p$ does not divide the order of the Weyl group of the overlying 
algebraic group, then, inductively, the situation is well behaved (see (2.3), (2.5), (2.6), and (2.7)(iv)). The more difficult cases are when $p$ is small or when $p$ is large with $m_{p}(E)=2$. When generation fails to hold the group $E$ typically has large $p$-rank, though not necessarily equal to the $p$-rank of $G$.

We make the following additional remarks concerning the proof of Theorem 1. The classical groups are handled using the standard module together with elementary facts on generation. For the exceptional groups we use more machinery. We introduce the algebraic group, $\bar{G}$, giving rise to $G$ in order to get information on centralizers. In fact, we pass to a universal group where centralizers are better behaved. In $\$ 3$ we use $\bar{G}$ to prove the existence of certain subgroups of $G$ that contain $E$. The tool for this is Lang's theorem, and as a result we obtain many of the embeddings that Stensholt gets in [26]. However we need a bit more than his results give. The generation is then proved by using induction and classification theorems of Aschbacher [2] and Timmesfeld [27].

For the most part our notation is standard. $E_{p^{n}}$ denotes the elementary abelian group of order $p^{n}$. For $X$ a group, let $E(X)$ denote the join of all quasisimple subnormal subgroups of $X$. We also make use of the following abuse of notation. Let $G$ be a group with $X \subseteq G$ and $Y, Z \leqslant G$. By $X^{Y} \cap Z$ we mean the set of $Y$-conjugates of $X$ contained in $Z$. The term Chevalley group refers to a group of Lie type generated by its root subgroups.

The author would like to thank R. Lyons for many helpful suggestions.

2. Notation and preliminary lemmas. We will use the following notation. Let $G=G(q)$ be a simple Chevalley group (normal or twisted) defined over a field $\mathbf{F}_{q}$ of characteristic $r$, and let $\bar{G}$ be the overlying algebraic group defined over the algebraic closure, $K$, of $\mathbf{F}_{r}$. Then $G=O^{r^{\prime}}\left(\bar{G}_{\sigma}\right)$ for $\sigma$ an endomorphism of $\bar{G}$ satisfying $\left|\bar{G}_{\sigma}\right|<\infty$. Fix a prime $p \neq r$ and a subgroup $E \cong E_{p^{n}}$, for $n>1$, of $\bar{G}_{\sigma}$. So $E$ is in the subgroup of $\operatorname{Aut}(G)$ generated by inner and diagonal automorphisms.

As centralizers are better behaved in universal covering groups we introduce the following groups. $\hat{G}$ denotes the universal covering group of $\bar{G}$ (see [25]). Then $\sigma$ induces an endomorphism of $\hat{G}$, again called $\sigma$, and $G_{0}=\hat{G}_{\sigma}$ is a central extension of $G$. Let $E_{0}$ denote the Sylow $p$-subgroup of the preimage in $\hat{G}$ of $E$. Notice that $E_{0}$ may not be abelian and $E_{0}$ need not be in $\hat{G}_{\sigma}=G_{0}$.

If $Y$ acts on a group $X$ let $C_{X}^{0}(Y)=\left\langle C_{X}\left(Y_{1}\right): Y_{1}\right.$ maximal in $\left.Y\right\rangle$ and $C_{X}^{r}(Y)=$ $\left\langle O^{r^{\prime}}\left(C_{X}\left(Y_{1}\right)\right): Y_{1}\right.$ maximal in $\left.Y\right\rangle$. Set $D=C_{G}^{0}(E)$ and $D_{0}=C_{G_{0}}^{0}\left(E_{0}\right)$. The first observation is

(2.1) (i) $D_{0}=G_{0}$ implies $D=G$.

(ii) $C_{G_{0}}^{r}\left(E_{0}\right)=G_{0}$ implies $C_{G}^{r}(E)=G$.

Proof. This follows easily by taking homomorphic images.

In view of (2.1) we work primarily with $G_{0}$. The $(B, N)$-pair notation for $G_{0}$ will be standard. Let $B_{0}$ be a fixed Borel subgroup of $G_{0}$ with $B_{0}=U H_{0}$, where $H_{0}$ is a Cartan subgroup and $U=O_{r}\left(B_{0}\right)$. The image of $B_{0}$ in $G$ is $B$. Notice that $U \cong U Z\left(G_{0}\right) / Z\left(G_{0}\right)$. $\Sigma$ denotes a root system for $G_{0}$, so $U=\prod_{\alpha \in \Sigma^{+}} U_{\alpha}$, a product of root subgroups. Let $\Pi=\left\{\alpha_{1}, \ldots, \alpha_{n}\right\}$ be a fundamental set of roots for $\Sigma$, let 
$N_{0} / H_{0}=W$, the Weyl group of $G_{0}$, and choose fundamental reflections $s_{i} \in\left\langle U_{\alpha_{i}}, U_{-\alpha_{i}}\right\rangle$ for $i=1, \ldots, n$ in the usual way.

In the group $\hat{G}$ we use similar notation, getting subgroups $\hat{B}, \hat{H}, \hat{U}, \hat{N}, \hat{U}_{\alpha}$, etc. The root system $\hat{\Sigma}$ may differ from that of $\Sigma$ and we will not always assume that $B_{0} \leqslant \hat{B}$ or that $\hat{B}$ is $\sigma$-invariant. Similarly, for $\bar{G}$.

If $P$ is a parabolic subgroup of $G_{0}$ with $B_{0} \leqslant P<G_{0}$ we define the opposite parabolic subgroup, $P_{1}$, as follows. Write $P=O_{r}(P) L$, where $L$ is the Levi factor of $P$ and $O_{r}(P)$ is a product of root subgroups for positive roots. Then $P_{1}=O_{r}\left(P_{1}\right) L$, where $O_{r}\left(P_{1}\right)$ is the product of those root subgroups $U_{\alpha}$, such that $U_{-\alpha} \leqslant O_{r}(P)$.

(2.2) Let $B_{0} \leqslant P<G_{0}$ be a proper parabolic subgroup of $G_{0}$ and let $P_{1}$ be the opposite parabolic subgroup. Then $G_{0}=\left\langle O_{r}(P), O_{r}\left(P_{1}\right)\right\rangle$.

Proof. Say $P=\left\langle B_{0}, s_{i_{1}}, \ldots, s_{i_{k}}\right\rangle$. Then $P=O_{r}(P) L$ where

$$
L=H_{0}\left\langle U_{ \pm \alpha_{i}}: i=i_{1}, \ldots, i_{k}\right\rangle \text {. }
$$

Moreover $O_{r}(P)$ is the product of those root subgroups $U_{\alpha} \leqslant U$ such that the coefficient of $\alpha_{i}$ in $\alpha$ is positive for some $i \in\{1, \ldots, n\}-\left\{i_{1}, \ldots, i_{k}\right\}$. Also $P_{1}=O_{r}\left(P_{1}\right) L$ with $O_{r}\left(P_{1}\right)$ the product of the root subgroups $U_{-\alpha}$ for roots $\alpha \in \Sigma$ with $U_{\alpha} \leqslant O_{r}(P)$.

Let $X=\left\langle O_{r}(P), O_{r}\left(P_{1}\right)\right\rangle$. Then $L \leqslant N(X)$ and, for $i \in\{1, \ldots, n\}-\left\{i_{1}, \ldots, i_{k}\right\}$, $\left\langle U_{ \pm \alpha_{i}}\right\rangle \leqslant X \leqslant N(X)$. As $G_{0}=\left\langle U_{ \pm \alpha_{i}}: i=1, \ldots, n\right\rangle$, we have $X \unlhd G_{0}$ and so $X=G_{0}$.

(2.3) Let $Y$ be a noncyclic elementary abelian $p$-subgroup in $\operatorname{Aut}\left(G_{0}\right)$ and suppose $Y$ normalizes a proper parabolic subgroup of $G_{0}$. Then $C_{G_{0}}^{r}(Y)=G_{0}$. In particular $C_{G_{0}}^{0}(Y)=G_{0}$.

Proof. Suppose $Y$ normalizes $P_{0} \geqslant B_{0}$. Then $C_{G_{0}}^{r}(Y) \geqslant O_{r}\left(P_{0}\right)$. By (2.2) it will suffice to show that, for some $g \in P_{0}, Y^{g}$ normalizes $P_{1}$, where $P_{1}$ is opposite to $P_{0}$. For then $C_{G_{0}}^{r}\left(Y^{g}\right)=G_{0}$ and we have the result. Consider $G Y \leqslant \operatorname{Aut}\left(G_{0}\right)$. Let $P=P_{0} / Z\left(G_{0}\right)$, so $Y \leqslant N(P)$. Now $N(P) \cap G Y=O_{r}(P) \bar{L}$, where $\bar{L} \cap G$ is the Levi factor, $L$, of $P$ and $\bar{L}$ is the extension of $L$ by certain combinations of diagonal, field, and graph automorphisms of $G$. It is easily verified that $\bar{L}$ stabilizes $P_{1} / Z\left(G_{0}\right)$. Since $p \neq r$ we have $Y$ conjugate in $P$ to a subgroup of $\bar{L}$, as needed.

Next we give several general results on centralizers of semisimple elements. What is involved is the statement of known results about centralizers in the algebraic group and the restrictions to the finite groups.

(2.4) (i) Let $x$ be a semisimple element in $\hat{G}$. Then $C_{\hat{G}}(x)=C_{\hat{G}}(x)^{0}$.

(ii) Let $x \in \bar{G}$ be semisimple. Then $C_{\bar{G}}(x) / C_{\bar{G}}(x)^{0}$ is isomorphic to a subgroup of $Z(\hat{G})$.

(iii) Let $x \in \bar{G}$ be semisimple and suppose $x$ has finite order with $(|x|,|Z(\hat{G})|)=$ 1. Then $C_{\vec{G}}(x)=C_{\vec{G}}(x)^{0}$.

Proof. (i) and (ii) follow from (4.4) on p. 204 of [22], while (iii) follows from (4.5) (again p. 204 of [22]).

(2.5) Let $x \in G_{0}$ (or, $x \in G$ with $(|x|,|Z(\hat{G})|)=1$ ) be semisimple. Then $C_{\hat{G}}(x)$ (respectively, $\left.C_{\vec{G}}(x)\right)$ is the central product of $E\left(C_{\hat{G}}(x)\right)$ (respectively, $E\left(C_{\vec{G}}(x)\right)$ ) and a torus. Also, $C_{G_{0}}(x)$ (respectively, $C_{G}(x)$ ) contains a normal subgroup, $X$, where $X$ is a central product of Chevalley groups (normal or twisted) with an abelian 
$r^{\prime}$-subgroup. $C_{G_{0}}(x)$ (respectively, $\left.C_{G}(x)\right)$ normalizes each of the factors of $X$ and induces inner and diagonal automorphisms on each factor. In particular, $O^{r^{\prime}}(X)=$ $O^{r^{\prime}}\left(C_{G_{0}}(x)\right)$.

Proof. Consider $C_{\hat{G}}(x)_{\sigma}$ (respectively $\left.C_{\bar{G}}(x)_{\sigma}\right)$ and apply (2.4).

(2.6) Let $x$ and $X$ be as in (2.5).

(i) $O_{r^{\prime}}\left(C_{G_{0}}(x)\right) \leqslant Z\left(C_{G_{0}}(x)\right)$ (respectively $O_{r^{\prime}}\left(C_{G}(x)\right) \leqslant Z\left(C_{G}(x)\right)$ ).

(ii) $O^{r^{\prime}}(X)$ is a central product of Chevalley groups each defined over some extension field of $\mathbf{F}_{q}$.

Proof. (i) is clear from (2.5). For (ii) assume that $L$ is a component of $C_{\hat{G}}(x)$ (respectively $C_{G}^{-}(x)$ ) and let $\left\{L=L_{1}, \ldots, L_{k}\right\}$ be the orbit of $L$ under $\langle\sigma\rangle$. Then $\sigma^{k}=\delta$ normalizes each of $L_{1}, \ldots, L_{k}$. It will suffice if we can show that $O^{r^{\prime}}\left(L_{\delta}\right)$ is a Chevalley group (normal or twisted) defined over $\mathbf{F}_{q^{k}}$. Suppose $Y=Y^{\delta}$ for a root subgroup, $Y$, of $\hat{G}$ with $Y \leqslant L$. It will suffice, in this case, to show $\left|Y_{\delta}\right|=q^{k}$. Say $Y=\hat{U}_{\alpha}^{g}$, where $\alpha \in \hat{\Sigma}$ and $g \in \hat{G}$. Then $g \delta g^{-1} \in N\left(\hat{U}_{\alpha}\right) \cap \hat{G} \delta$. As $\hat{G}_{\sigma}$ is defined over $\mathbf{F}_{q}, \hat{G}_{\delta}$ is defined over $\mathbf{F}_{q^{k}}$ and there is a conjugate $\tau$ of $\delta$ with $\tau \in N\left(\hat{U}_{\alpha}\right)$ and $\left|\left(\hat{U}_{\alpha}\right)_{\tau}\right|=q^{k}$. We have $g \delta g^{-1} \in \hat{G} \tau$, say $g \delta g^{-1}=y \tau$ for $y \in \hat{G}$. Then $y \in N_{\hat{G}}\left(\hat{U}_{\alpha}\right)$, so $y$ acts on $\hat{U}_{\alpha}$ as multiplication by a scalar. It now follows from (11.2) of [23] that $\left|\hat{U}_{\alpha} \cap C(y \tau)\right|=q^{k}$, and so $\left|Y_{\delta}\right|=q^{k}$, as needed. If no such $Y$ exists, then $\hat{G}=F_{4}(K), S p(4, K)$, or $G_{2}(K)$ and $\delta^{2}$ fixes a root subgroup of $L$. Here $\sigma^{2}$ is a field automorphism of $\hat{G}$, with $\hat{G}_{\sigma^{2}}$ defined over $\mathbf{F}_{q}$ and $\sigma$ a graph automorphism of $\hat{G}_{\sigma^{2}}$. Argue as above with $\delta^{2}$ and $\sigma^{2}$, and the result follows.

(2.7) (i) $E_{0}$ and $E$ normalize maximal tori of $\hat{G}$ and $\bar{G}$, respectively.

(ii) If $p \nmid|\hat{W}|$, then $E_{0}$ is contained in a maximal torus of $\hat{G}$. Similarly for $E \leqslant \bar{G}$.

(iii) If $(p,|Z(\hat{G})|)=1$ and if $E \geqslant F \cong Z_{p} \times Z_{p}$, then $F$ is contained in a maximal torus of $\bar{G}$.

(iv) If $E$ contains a subgroup $F \cong E_{p^{m}}$ for $m>1$ with $F$ in a maximal torus of $\bar{G}$, then some maximal subgroup of $F$ is contained in a proper parabolic subgroup of $\bar{G}_{\sigma}$.

(v) If $\alpha \in \hat{\Sigma}$ is a long root and $g \in \hat{G}$ such that $\hat{U}_{\alpha}^{g \sigma}=\hat{U}_{\alpha}^{g}$, then $\left(\hat{U}_{\alpha}^{g}\right)_{\sigma}$ is a long root subgroup of $G_{0}$.

Proof. Consider $E \leqslant \bar{G}$. The reference for (i), (ii) and (iii) is Springer-Steinberg [22]. Namely, (i) is a consequence of (5.17), (ii) follows from (i), and (iii) follows from (5.1). To get (iv) assume that $F$ is as given with $F \leqslant \bar{H}$. Then $F$ normalizes each root subgroup $\bar{U}_{\alpha}$ for $\alpha \in \bar{\Sigma}$, inducing a cyclic group on $\bar{U}_{\alpha}$ (as $\bar{H}$ induces a 1-dimensional torus on $\bar{U}_{\alpha}$ ). So there is a root $\alpha \in \bar{\Sigma}^{+}$and hyperplane $F_{0}<F$ with $F_{0} \leqslant C_{G}\left(\bar{U}_{\alpha}\right)$. Then $F_{0} \leqslant C\left(\left\langle\bar{U}_{ \pm \alpha}\right\rangle\right)$ and $C_{G}\left(F_{0}\right)$ contains a nontrivial component. Since $\sigma$ normalizes $C_{G}\left(F_{0}\right), C_{G}\left(F_{0}\right)_{\sigma}$ contains a nontrivial $r$-subgroup, say $I$. Then $F_{0} \leqslant C_{G}(I)$ which is contained in a proper parabolic subgroup of $\bar{G}_{\sigma}$ (Borel-Tits [6]). (v) follows from Lang's theorem $\left((10.1)\right.$ of [23]) and the fact that $N_{\hat{G}}\left(\hat{U}_{\alpha}\right)$ is connected.

The next result contains the application of the work of Aschbacher [2] and Timmesfeld [27].

(2.8) Assume $G \neq^{2} F_{4}(q)^{\prime}$ and $(p, q) \neq(3,2)$. Let $V$ be the center of a long root subgroup of $G_{0}$ with $V \leqslant D_{0}$. Set $X=\left\langle V^{G_{0}} \cap D_{0}\right\rangle$. Then one of the following holds: 
(i) $И\left(r, E_{0}\right) \neq\{1\}$ and $D_{0}=G_{0}$.

(ii) $X / Z(X)$ is a direct product of groups of Lie type each defined over $\mathbf{F}_{q}$, and each component of $X$ is generated by conjugates of $V$. If $X_{1}$ is a component of $X$, then $V^{G} \cap X_{1}$ consists of the centers of long root subgroups of $X_{1}$ (or possibly short root subgroups if $X_{1} / Z\left(X_{1}\right) \cong P \operatorname{Sp}\left(4,2^{b}\right), F_{4}\left(2^{b}\right)$, or $\left.G_{2}\left(3^{b}\right)\right)$.

(iii) $r=3,|V|=3$, and setting $\bar{X}=X / O_{2}(X), \bar{X} / Z(\bar{X})$ is as in (ii).

(iv) $r=2,|V|=2$, and setting $\bar{X}=X / O_{3}(X), E(\bar{X} / Z(\bar{X}))$ is a central product $Y_{1} \cdots Y_{k}$, where, for $i=1, \ldots, k, Y_{i} \unlhd \bar{X}$ and $Y_{i}$ is either a Chevalley group over $\mathbf{F}_{2}$ (as in (ii)) or $E\left(Y_{i}\right)$ is one of the following: $P S O^{ \pm}(n, 2)^{\prime}, P S O^{ \pm}(n, 3)^{\prime}, A_{n}, F_{22}, F_{23}$, $F_{24}^{\prime}$. Also, $\bar{X} / E(\bar{X})$ is solvable.

Proof. Suppose (i) is false. By (2.3) we have $И\left(r, E_{0}\right)=\{1\}$. In particular $O_{r}(X)=1$. Let $O_{\infty}(X)$ be the solvable radical of $X$ and set $Y=O_{\infty}(X) V_{1}$, where $V_{1} \in V^{G} \cap X$. For $g \in G_{0}$ arbitrary, $\left\langle V_{1}, V_{1}^{g}\right\rangle$ is either an $r$-group or isomorphic to $S L(2, q)$ (see (12.1) of [3]). Suppose $\left|V_{1}\right|>3$. Then $\left\langle V_{1}, V_{1}^{y}\right\rangle$ is an $r$-group for each $y \in Y$, and we conclude that $V_{1} \leqslant O_{r}(Y)$ (see [1]). But $O_{r}\left(O_{\infty}(X)\right) \leqslant O_{r}(X)=1$ and hence $\left[V_{1}, O_{\infty}(X)\right]=1$. We then conclude that $O_{\infty}(X)=Z(X)$ for $q=\left|V_{1}\right|>3$.

Next suppose that $q=2$ or 3 . Then $V_{1}$ centralizes any chief factor of $Y$ having order prime to 6. If $s$ is a prime, then $O_{s^{\prime}, s}(Y)$ is the intersection of the centralizers in $Y$ of all $s$-chief factors of $Y$. Therefore $V_{1} \leqslant \cap{ }_{s} O_{s^{\prime}, s}(Y)=Y_{0}$, where the intersection is taken over all primes greater than 3. If $X_{0}=O_{\{2,3\}}\left(Y_{0} \cap O_{\infty}(X)\right)$ and $R \in \operatorname{Syl}_{r}\left(X_{0}\right)$, then $X=X_{0} N_{X}(R)$. Since $p \neq 2,3$, this contradicts $И\left(r, E_{0}\right)=\{1\}$ unless $R=1$. Therefore $R=1$ and $X_{0}=O_{3}(X)$ or $O_{2}(X)$ according to whether $q=2$ or 3 . In addition, $V_{1}$ centralizes $O_{\infty}(X) / X_{0}$ for each $V_{1} \in V^{G} \cap X$, and so $X$ centralizes $O_{\infty}(X) / X_{0}$.

Let $Y=X / Z(X)$ if $|V|>3$. If $|V|=2$ or 3 , then set $Y=\bar{X} / Z(\bar{X})$, where $\bar{X}=X / O_{3}(X)$ or $X / O_{2}(X)$, respectively. Let $I=F^{*}(Y)$, so that $I=E(Y)$ (by the above). Write $I=I_{1} \cdots I_{k}$, a central product of components. For $V_{1} \in V^{G} \cap X, V_{1}$ permutes $\left\{I_{1}, \ldots, I_{k}\right\}$, and the above property of $V_{1}$ forces $V_{1} \leqslant N\left(I_{j}\right)$ for $j=1, \ldots, k$. Therefore, $I_{j} \unlhd Y$ for $j=1, \ldots, k$.

Fix $j=1, \ldots, k$ and choose $V_{1} \in V^{G} \cap X$ such that $\left[I_{j}, V_{1}\right] \neq 1$. Let $\tilde{V}_{1}$ denote the image of $V_{1}$ in $Y$ and set $J=I_{j} \tilde{V}_{1}$. Then $J^{\prime \prime}=J^{\prime}=I_{j}=F^{*}(J)$. If $q$ is even we can apply Theorem 1 of [27] to determine the structure of $J$. If $q$ is odd apply (4.2) of [29] to see that for $j \in J$ with $K=\left\langle\tilde{V}_{1}, \tilde{V}_{1}^{j}\right\rangle \cong S L_{2}(q)$, the class $K^{J}$ satisfies Hypothesis $\Omega$ in [2]. Therefore, Theorem 1 of [2] applies and yields the structure of $I_{j}$ (we note that $D_{4}(2)$ should be included in Theorem 1 of [2], but this is not a possibility here due to the fact that $K \cong S L_{2}(q)$ ).

We conclude from the above that either $I_{j}$ is a group of Lie type in characteristic $r$ and $\tilde{V}_{1}^{\#}$ consists of elements of long root subgroups of $J$ (possibly short root subgroups if $I_{j}$ is isomorphic to $\operatorname{PSp}\left(2 n, 2^{b}\right), F_{4}\left(2^{b}\right)$, or $\left.G_{2}\left(3^{b}\right)\right)$ or $q=2$ and one of the following holds:

(i) $J \cong O^{ \pm}\left(n, 2^{b}\right)$ and $v \in \tilde{V}_{1}^{\#}$ is a transvection;

(ii) $J \cong O^{ \pm}(n, s), s=3$ or 5 , and $v \in \tilde{V}_{1}^{\#}$ is a reflection;

(iii) $J \cong S_{n}$ and $v \in \tilde{V}_{1}^{\#}$ is a transposition; or

(iv) $J \cong A_{6}, J_{2}, F_{22}, F_{23}$, or $F_{24}$. 
If $q=2$, then for $v \in \tilde{V}_{1}^{\#}, r^{J}$ is a class such that for $v_{1}, v_{2} \in v^{J},\left|v_{1} v_{2}\right|=1,2,3$, or 4. So by the main results of Fischer [11] and Timmesfeld [27] we see that the cases $J_{2}$ and $O^{ \pm}(n, 5)$ do not occur. At this stage we may assume that $J$ is a group of Lie type in characteristic $r$ and the elements of $\tilde{V}_{1}^{\#}$ are long root elements of $J$ (possibly short root elements in the three exceptional cases).

We claim that $\tilde{V}_{1}$ is contained in the center of a root subgroup of $J$. This is trivial if $\left|\tilde{V}_{1}\right|=r$ or if $J$ has Lie rank 1. So suppose $\left|\tilde{V}_{1}\right|>r$ and $J$ has Lie rank at least 2. Since $\left|\tilde{V}_{1}\right|>r, Y=X / Z(X)$ and $V_{1} \leqslant J^{(\infty)}=J_{0}$, a component of $X$. If the claim is false, then there exist elements $a, b \in V_{1}^{\#}$ and centers of long root subgroups, $U_{1} \neq U_{2}$ of $J_{0}$, such that $a \in U_{1}$ and $b \in U_{2}$. Fix $g \in J_{0}$ such that $\left\langle U_{1}, U_{1}^{g}\right\rangle \cong S L_{2}\left(q_{1}\right)$, where $q_{1}=\left|U_{1}\right|$. As $\left\langle a, a^{g}\right\rangle$ is not an $r$-group, $\left\langle V_{1}, V_{1}^{g}\right\rangle \cong S L_{2}(q)$, and so $\left\langle b, b^{g}\right\rangle$ is not an $r$-group. Also, $O^{r^{\prime}}\left(C_{G}\left(\left\langle a, a^{g}\right\rangle\right)\right)=O^{r^{\prime}}\left(C_{G}\left(\left\langle V_{1}, V_{1}^{g}\right\rangle\right)\right)=O^{r^{\prime}}\left(C_{G}\left(\left\langle b, b^{g}\right\rangle\right)\right)$; in each case the centralizer has the form $O^{r^{\prime}}(L)$, where $L$ is a Levi factor of $C_{G}\left(V_{1}\right)$. Similarly, $E=O^{r^{\prime}}\left(C_{J_{0}}\left(\left\langle U_{1}, U_{1}^{g}\right\rangle\right)\right)=O^{r^{\prime}}\left(C_{J_{0}}\left(\left\langle b, b^{g}\right\rangle\right)\right)=O^{r^{\prime}}\left(C_{J_{0}}\left(\left\langle U_{2}, U_{2}^{g}\right\rangle\right)\right)$. But $\left\langle U_{1}, U_{1}^{g}\right\rangle=O^{r^{\prime}}\left(C_{J_{0}}(E)\right)=\left\langle U_{2}, U_{2}^{g}\right\rangle$. Since $[a, b]=1$, we must have $U_{1}=U_{2}$, a contradiction.

Finally, we must prove that $\tilde{V}_{1}$ is the center of a long root subgroup of $J$. If not, then the previous paragraph implies the existence of a group $U>\tilde{V}_{1}$ with $U$ the center of a long root subgroup of $J$. Choose $j \in J$ such that $\left\langle U, U^{j}\right\rangle \cong S L_{2}\left(q_{1}\right)$, where $q \mid q_{1}$ and $q_{1}=|U|$. If $r=2$, then we see that for $a \in \tilde{V}_{1}^{\#}$, there exists $j_{1} \in J$ such that $\left|a a^{j_{1}}\right|=q_{1}+1$. But this contradicts the fact that $\left\langle V_{1}, V_{1}^{g}\right\rangle$ is an $r$-group or $S L_{2}(q)$ for each $g \in G$. Suppose $r$ is odd. Then Dickson's theorem ((2.8.4) of [30]) implies that for suitable $j_{1} \in J$ either $\left\langle\tilde{V}_{1}, \tilde{V}_{1}^{j_{1}}\right\rangle \geqslant S L\left(2, q_{1}\right)$ or $q_{1}=9$ and $\left\langle\tilde{V}_{1}, \tilde{V}_{1}^{j_{1}}\right\rangle \cong S L_{2}(5)$. This is a contradiction.

(2.9) Let $E \leqslant F \leqslant G$ with $F$ an abelian $p$-group, and let $F_{0}$ be the Sylow $p$-subgroup of the preimage in $G_{0}$ of $F$.

(a) $C_{G}^{0}(F) \leqslant C_{G}^{0}(E)$ and $C_{G_{0}}^{0}\left(F_{0}\right) \leqslant C_{G_{0}}^{0}\left(E_{0}\right)$.

(b) $C_{G}^{r}(F) \leqslant C_{G}^{r}(E)$ and $C_{G_{0}}^{r}\left(F_{0}\right) \leqslant C_{G_{0}}^{r}\left(E_{0}\right)$.

Proof. Let $F_{1}$ be maximal in $F$. Then either $E \leqslant F_{1}$ or $E \cap F_{1}$ is maximal in $E$. In either case $C_{G}\left(F_{1}\right) \leqslant C_{G}\left(E \cap F_{1}\right) \leqslant C_{G}\left(E_{1}\right)$ for some maximal subgroup $E_{1}$ of $E$. The lemma follows.

(2.10) Let $X=X_{1} \cdots X_{k} \leqslant G_{0}$ be a product of pairwise commuting groups of Lie type each defined over a field of characteristic $r$. Let $Y \leqslant X$ be an elementary abelian $p$-group and $(p,|Z(X)|)=1$. Suppose that $Y$ projects onto a cyclic subgroup, $Y_{i}$, of $X_{i} / Z\left(X_{i}\right)$, for $i=1, \ldots, k$. Then

(a) $X \leqslant C_{G_{0}}^{0}(Y)$.

(b) If $r$ divides $\left|C_{X_{i}}\left(Y_{i}\right)\right|$ for some $i \in\{1, \ldots, k\}$, then $C_{G_{0}}^{r}(Y)=G_{0}$.

Proof. (a) follows by looking at suitable hyperplanes of $Y$-namely, the kernels of the projection maps. (b) follows from (2.3) and (3.12) of [6].

(2.11) Suppose $E \cong Z_{p} \times Z_{p}$, and $E_{0}$ is contained in the maximal torus $\hat{H}$ of $\hat{G}$, where $\hat{B}=\hat{U} \hat{H}$ is a Borel subgroup of $\hat{G}$ and $\hat{U}=\hat{B}_{u}$ (unipotent radical). Then there exist at least three maximal subgroups of $E_{0}$ with nontrivial centralizers in $\hat{U}$.

Proof. Suppose false. Then clearly $E_{0}$ centralizes no element of $\hat{U}^{\#}$ and we may then write $E_{0}=E_{1} E_{2}$ where, for $i=1,2, E_{i}$ contains $E_{0} \cap Z\left(G_{0}\right)$ as a subgroup of 
index $p$ and no element of $E_{0}-\left(E_{1} \cup E_{2}\right)$ centralizes an element of $\hat{U}^{\#}$. Let $\Delta_{i}=\left\{\alpha \in \hat{\Sigma}: E_{i} \leqslant C\left(\hat{U}_{\alpha}\right)\right\}$. As $E_{0}$ induces $Z_{p}$ on each root subgroup $\hat{U}_{\alpha}$ for $\alpha \in \hat{\Sigma}$ we have $\hat{\Sigma}=\Delta_{1} \cup \Delta_{2}$, a disjoint union. Also $\Delta_{1} \neq \varnothing \neq \Delta_{2}$ for otherwise $E_{i} \leqslant C(\hat{G})$ for $i=1$ or 2 . Finally we observe that $\Delta_{i}=-\Delta_{i}$ for $i=1,2$.

We claim that $\alpha \in \Delta_{1}$ and $\beta \in \Delta_{2}$ implies $\left[\hat{U}_{\alpha}, \hat{U}_{\beta}\right]=1$. Otherwise $\alpha+\beta \in \hat{\Sigma}$ and $\hat{U}_{\alpha+\beta} \leqslant\left[\hat{U}_{\alpha}, \hat{U}_{\beta}\right]$ (this follows from the commutator relations). Now fix $e \in E_{1}-$ $Z(\hat{G})$. Then $e$ is trivial on $\hat{U}_{\alpha}$, nontrivial on $\hat{U}_{\beta}$, and hence nontrivial on $\hat{U}_{\alpha+\beta}$. Consequently $\alpha+\beta \notin \Delta_{1}$. Similarly $\alpha+\beta \notin \Delta_{2}$, a contradiction. This proves the claim.

Let $\hat{G}_{i}=\left\langle\hat{U}_{\alpha}: \alpha \in \Delta_{i}\right\rangle$ for $i=1,2$. Then $\left[\hat{G}_{1}, \hat{G}_{2}\right]=1, \quad \hat{G}_{1} \hat{G}_{2}=\hat{G}$, and $\hat{G}_{1} \cap \hat{G}_{2} \leqslant Z(\hat{G})$. But this gives a direct decomposition of the simple group $\hat{G} / Z(\hat{G})$, which is impossible.

(2.12) Let $T$ be any maximal torus of $\bar{G}$ with $T=T^{\sigma}$. Then $\bar{G}_{\sigma}=O^{r^{\prime}}\left(\bar{G}_{\sigma}\right) T_{\sigma}$.

Proof. The following is based on a suggestion of G. Lustig. Let $\pi: \hat{G} \rightarrow \bar{G}$ be the natural surjection and regard $\sigma$ as an endomorphism of $\hat{G}$. Let $\hat{T}$ be the preimage in $\hat{G}$ of $T$ and let $Z=Z(\hat{G})$. Then $\hat{T}$ is connected (see (3.2) and (8.2) of [25]). Write $X=\hat{G} \times{ }_{Z} \hat{T}$ for the central product of $\hat{G}$ and $\hat{T}$ (amalgamation with respect to $Z$ ). Then $\sigma$ induces an endomorphism of $X$, via $(\hat{g}, \hat{t})^{\sigma}=\left(\hat{g}^{\sigma}, \hat{t}^{\sigma}\right)$. Also $X=X^{0}$ and there is a well-defined homomorphism (of algebraic groups) given by $(\hat{g}, \hat{t}) \theta=(\hat{g}) \pi$. Then $\operatorname{ker}(\theta)=\{(1, \hat{t}): \hat{t} \in \hat{T}\}$. Now $\operatorname{ker}(\theta) \cong \hat{T}$ is connected, so Lang's theorem ([18] or $(10.1)$ of [23]) implies that $\left(X_{\sigma}\right) \theta=\bar{G}_{\sigma}$.

Next we note that $\left(\hat{G}_{\sigma}\right) \pi=O^{r^{\prime}}\left(\bar{G}_{\sigma}\right)$ (see (3.2) of [25] and (12.4) of [23]). Hence $\left(\hat{T}_{\sigma}\right) \pi=T_{\sigma} \cap O^{r^{\prime}}\left(\bar{G}_{\sigma}\right)$. Let $g \in \bar{G}_{\sigma}-O^{r^{\prime}}\left(\bar{G}_{\sigma}\right)$ and suppose that $\left(\hat{g}_{1}, \hat{t}\right) \theta=g$ with $\left(\hat{g}_{1}, \hat{t}\right) \in X_{\sigma}$. Then, for some $z \in Z, \hat{g}_{1}^{\sigma}=\hat{g}_{1} z^{-1}$ and $\hat{t}^{\sigma}=\hat{t z}$. As the cosets $g O^{r^{\prime}}\left(\bar{G}_{\sigma}\right)$ vary, so does the element $z \in Z$. It follows that $\left|T_{\sigma} /\left(T_{\sigma} \cap O^{r^{\prime}}\left(\bar{G}_{\sigma}\right)\right)\right|=\left|\bar{G}_{\sigma} / O^{r^{\prime}}\left(\bar{G}_{\sigma}\right)\right|$, proving the lemma.

(2.13) Let $G_{1}=G_{1}^{0}$ be a quasisimple algebraic group over $K$ and $\sigma$ an endomorphism of $G_{1}$ with $\left|\left(G_{1}\right)_{\sigma}\right|$ finite. Let $G_{2}$ be the universal covering group of $G_{1}$ with $Z \leqslant Z\left(G_{2}\right)$ and $G_{2} / Z \cong G_{1}$. View $\sigma$ as an endomorphism of $G_{2}$ (see (9.16) of [23]). Let $X=\left\{g \in G_{2}: g^{\sigma}=g z\right.$ for some $\left.z \in Z\right\}$. Then $X$ is a group, $X \geqslant\left(G_{2}\right)_{\sigma} Z$, and $X /\left(G_{2}\right)_{\sigma} \cong Z$.

Proof. Let $X=\left\{g \in G_{2}: g^{\sigma}=g z\right.$ for some $\left.z \in Z\right\}$. Clearly $X$ is a subgroup containing $Z$ and $\left(G_{2}\right)_{\sigma}$. The map $x \rightarrow x^{-1} x^{\sigma}$ is a homomorphism from $X$ into $Z$. By Lang's theorem the map is surjective, so $X /\left(G_{2}\right)_{\sigma} \cong Z$.

(2.14) Let notation be as above and assume that $G_{1}$ is a simple group. Let $\pi$ be the natural surjection from $G_{2}$ to $G_{1}$. Then $\left(G_{1}\right)_{\sigma}=X^{\pi}$ and $\mid\left(G_{1}\right)_{\sigma}$ : $O^{r^{\prime}}\left(\left(G_{1}\right)_{\sigma}\right)|=|(Z)_{\sigma} \mid$.

Proof. Clearly $X^{\pi}=\left(G_{1}\right)_{\sigma}$. Now set $X_{0}=\left(G_{2}\right)_{\sigma} Z$. Then $X_{0} /\left(G_{2}\right)_{\sigma} \cong Z / Z_{\sigma}$, so $\left|X / X_{0}\right|=\left|Z_{\sigma}\right|$. As $X_{0}$ is the preimage under $\pi$ of $O^{r^{\prime}}\left(\left(G_{1}\right)_{\sigma}\right)$, the result follows.

(2.15) Let $G_{1}$ be as in (2.14). Then $\left(G_{1}\right)_{\sigma}$ is the group $O^{r^{\prime}}\left(\left(G_{1}\right)_{\sigma}\right)$ together with all diagonal automorphisms. Moreover $\left(G_{1}\right)_{\sigma} / O^{r^{\prime}}\left(\left(G_{1}\right)_{\sigma}\right)$ is isomorphic to an image of $Z$ of order equal to $\left|Z_{\sigma}\right| .\left(G_{2}\right)_{\sigma}$ is a universal covering group of $O^{r^{\prime}}\left(\left(G_{1}\right)_{\sigma}\right)$.

Proof. For the last statement see 12.8 of [23]. For the second statement use (2.14) and (12.6) of [23]. The first statement follows from (12.3) and (11.6) of [23]. 
The following is useful in inductive situations and for various generalizations of the main theorems.

(2.16) Let $X=X_{1} \cdots X_{n}$ be a central product of quasisimple groups and let $E$ be an elementary abelian $p$-group acting on $X$. For each $i=1, \ldots, n$ assume that $C_{X_{i}}^{0}\left(E_{i}\right)=X_{i}$, where $E_{i}$ is the stabilizer in $E$ of $X_{i}$. Then $C_{X}^{0}(E)=X$.

Proof. We may assume that $E$ is transitive on $\left\{X_{1}, \ldots, X_{n}\right\}$, so $E_{i}=E_{j}$ for each $i, j$ and we set $F=E_{1}$. Clearly we may also assume $n>1$. Let $I$ be a maximal subgroup of $E$ with $F \leqslant I$. Write $X=Y_{1} \cdots Y_{p}$, where each $Y_{i}$ is the product of the components of $X$ in a fixed $I$-orbit. Inductively, $Y_{i}=C_{Y_{i}}^{0}(I)$ for $i=1, \ldots, k$.

Fix $e \in E-I$ and let $J$ be any maximal subgroup of $I$. Then for $y \in C_{Y_{1}}(J)$, we have $\prod_{i=0}^{p-1} y^{e^{i}}$ an element of $C_{X}(J\langle e\rangle)$. Do this for each maximal subgroup of $I$ and conclude that $C_{X}^{0}(E)$ covers $C_{X / Z(X)}(e)$, so contains $C_{X}(e)^{\prime}$. Now this happens for each $e \in E-I$. It follows that $C_{X}^{0}(E)=X$, as required.

Throughout this paper we will use the following labeling of Dynkin diagrams.

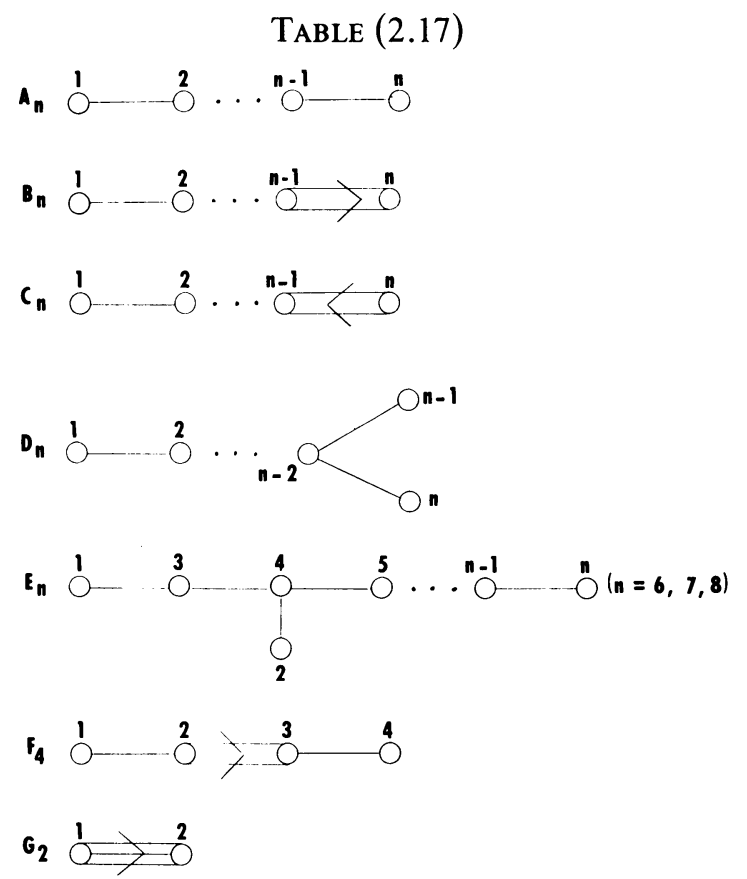

3. Subgroups of Chevalley groups. In this section we prove some results on generation of classical groups that will be used in $\$ 4$. Also we establish the existence of certain subgroups of some exceptional groups of Lie type. These subgroups are similar to ones produced by Stensholt in [26]. However our methods are different, and yield a bit of additional information that we require in $\$ 5$.

Let $W$ be an $\mathbf{F}_{q}$-space with a nondegenerate alternating or hermitian bilinear form, or quadratic form in case $W$ is an orthogonal space. Say $X$ is a subspace of $W$ with $W=X \oplus X^{\perp}$. Define $9(X)$ to be the group of isometries of $W$ generated by those root subgroups trivial on $X^{\perp}$. For convenience there will be two cases where we vary from this definition, namely, the case when $W$ is a unitary space with $\operatorname{dim}(X)=1$ 
and $W$ an orthogonal space with $\operatorname{dim}(X)=2$. In the first case we let $9(X)$ be the group of all isometries of $W$ that are trivial on $X^{\perp}$. So $\mathscr{G}(X) \cong Z_{q+1}$. In the second case $\mathscr{G}(X)$ will be the subgroup of $S O(W)$ that is trivial on $X^{\perp}$. So here $\mathscr{G}(X) \cong Z_{q \pm 1}$, depending on whether $X$ is anisotropic or not.

(3.1) Assume $W=W_{1} \perp W_{2} \perp W_{3}$ and that $W_{2}$ contains nonzero isotropic (singular if $W$ is an orthogonal space) vectors. Then either

(i) $\mathscr{G}(W)=\left\langle 9\left(W_{1} \perp W_{2}\right), 9\left(W_{2} \perp W_{3}\right)\right\rangle$, or

(ii) $9(W) \cong S O^{+}(6,2) \cong A_{8}$ and $\left\langle 9\left(W_{1} \perp W_{2}\right), 9\left(W_{2} \perp W_{3}\right)\right\rangle \cong A_{7}$.

Proof. Set $X=\left\langle 9\left(W_{1} \perp W_{2}\right), 9\left(W_{2} \perp W_{3}\right)\right\rangle$. It is easy to see that $X$ is transitive on each $9(W)$ orbit of $V$. Also we may assume $W_{1} \neq 0 \neq W_{3}$. The idea is to show that $X$ is flag-transitive in the sense of [21]. Once this is achieved we will be done by applying the main theorem of [21].

Suppose $W_{3}$ contains an isotropic (singular) 1-space $W_{0}$, and write $W_{3}=W_{4} \perp \bar{W}_{0}$, where $\bar{W}_{0}$ is a nondegenerate 2-space containing $W_{0}$. Let $P$ be the stabilizer in $9(W)$ of $W_{0}$. Then $P$ is a parabolic subgroup of $\mathscr{Q}(W)$ and the transitivity of $X$ implies that $G=P X$. Also $P=O_{r}(P) L H$, where $L=9\left(W_{1} \perp W_{2} \perp W_{4}\right)$ and $H$ is a Cartan subgroup of $9(W)$ normalizing $L$. We may take $O_{r}(P) H \leqslant B \leqslant P$, where $B$ is a Borel subgroup of $\mathscr{}(W)$.

In view of the main theorem of [21] it will suffice to show $B X=9(W)$, and for this we only need $P=B(P \cap X)$. However, inductively we have $L=9\left(W_{1} \perp W_{2} \perp W_{4}\right)=\left\langle 9\left(W_{1} \perp W_{2}\right), \quad 9\left(W_{2} \perp W_{4}\right)\right\rangle$ or $L \cong A_{8}$ and $\left\langle 9\left(W_{1} \perp W_{2}\right), \Phi\left(W_{2} \perp W_{4}\right)\right\rangle \cong A_{7}$. In either case we have $P=B(P \cap X)$. So we may now assume that $W_{3}$, and by symmetry $W_{1}$, contains no isotropic (singular) 1-spaces.

In particular $W$ is not a symplectic space. If $W$ is a unitary space, then $\operatorname{dim}\left(W_{1}\right)=\operatorname{dim}\left(W_{3}\right)=1$. In this case let $W_{4}$ be an isotropic l-space in $W_{1} \perp W_{3}$ and $P$ the stabilizer in $\mathscr{G}(W)$ of $W_{4}$. Then $P=O_{r}(P) \Phi\left(W_{2}\right) H$ and we have $P=B(X \cap P)$ for a Borel subgroup of $\mathscr{G}(W)$. As above this gives the result.

Now we may assume $W$ is an orthogonal space. Write $W_{2}=W_{2}^{\prime} \perp W_{2}^{\prime \prime}$, where $W_{2}^{\prime}$ is a 2-space containing a singular 1-space. Let $W_{3}^{\prime}=W_{2}^{\prime \prime} \perp W_{3}$ and consider the decomposition $W=W_{1} \perp W_{2}^{\prime} \perp W_{3}^{\prime}$. By the above arguments we may assume that $W_{3}^{\prime}$ contains no singular 1-space. So $\operatorname{dim}\left(W_{1}\right)$ and $\operatorname{dim}\left(W_{3}^{\prime}\right)$ are each at most 2.

If $\operatorname{dim}\left(W_{1}\right)=1$, then $W_{1}=\langle v\rangle$ for some nonisotropic vector, $v$, of $W$. Therefore $X$ contains $9\left(W_{2}^{\prime} \perp W_{3}^{\prime}\right)$ which is of index 2 in $C_{q_{(W)}}(v)$ and $X$ is transitive on $v^{q(W)}$. But then $X$ has index at most 2 in $\mathscr{T}(W)$, proving $X=\mathscr{}(W)$. So we may assume $\operatorname{dim}\left(W_{1}\right)=2$, and similarly $\operatorname{dim}\left(W_{3}^{\prime}\right)=2$.

If $q$ is odd we may write $W_{1}=W_{11} \perp W_{12}$ with $\operatorname{dim}\left(W_{11}\right)=\operatorname{dim}\left(W_{12}\right)=1$. Then argue as above, using the fact that $9\left(W_{12} \perp W_{2}^{\prime} \perp W_{3}^{\prime}\right) \leqslant X$ by induction. So assume $q$ is even. Let $W_{0}$ be a singular l-space in $W_{2}^{\prime}$ and $P$ the stabilizer in $9(W)$ of $W_{0}$. Then $P=O_{r}(P) L H$, where $O_{r}(P) H$ is contained in a Borel subgroup $B \leqslant P$ and $L=\mathscr{}\left(W_{1} \perp W_{3}^{\prime}\right)$. But $X \geqslant \mathscr{}\left(W_{1}\right) \times 9\left(W_{3}^{\prime}\right) \cong Z_{q+1} \times Z_{q+1}$ and we have $L=(L \cap B)\left(\mathscr{G}\left(W_{1}\right) \times \mathscr{(}\left(W_{3}^{\prime}\right)\right)$. So $B X=\mathscr{G}(W)$ and we use [21] to complete the proof.

(3.2) Let $V=V_{1} \perp V_{2}$ be an $\mathbf{F}_{q}$-space with a nondegenerate alternating or hermitian form, or a quadratic form. Assume $\operatorname{dim}\left(V_{1}\right) \geqslant \operatorname{dim}\left(V_{2}\right) \geqslant 2$, that $\operatorname{dim}\left(V_{1}\right) \geqslant 4$ if $V$ is 
an orthogonal space, $\operatorname{dim}\left(V_{2}\right) \geqslant 3$ if $V$ is orthogonal and $q=3$, and $\operatorname{dim}\left(V_{2}\right) \geqslant 4$ if $V$ is an orthogonal space and $q$ is even. Let $X=S p(V), S U(V)$, or $O^{ \pm}(V)$, whichever is appropriate, and let $I \leqslant X$ be a subgroup containing $C_{X}\left(V_{1}\right)$ and $C_{X}\left(V_{2}\right)$, but not stabilizing $\left\{V_{1}, V_{2}\right\}$. Then one of the following holds:

(i) $I=X$;

(ii) $X \cong S U(4,2)$; or

(iii) $X \cong O^{+}(8,2)$.

Proof. Suppose $X \neq S U(4,2)$ or $O^{+}(8,2)$. Let $A=C_{X}\left(V_{2}\right)$. If $X \cong O^{-}(8,2)$, we may assume $A \cong O^{-}(4,2)$. There exists $g \in I$ such that $A^{g} \$ N_{X}(A)$, and, for such a group $A^{g}$, choose an element $c \in A^{g}-N(A)$ with $c$ a transvection or reflection (in case $q$ is odd and $\left.X=O^{ \pm}(V)\right)$. Then set $V_{0}=[\langle A, c\rangle, V]$. We have $V_{1}<V_{0}$ and $\operatorname{dim}\left(V_{0}\right)=\operatorname{dim}\left(V_{1}\right)+1$.

Suppose $\operatorname{rad}\left(V_{0}\right) \neq 0$. Then $\operatorname{rad}\left(V_{0}\right)$, being $A$-invariant, is contained in $V_{2}$ and $V_{0}=V_{1} \perp \operatorname{rad}\left(V_{0}\right)$. As $\operatorname{rad}\left(V_{2}\right)=0$ there is a 2-space $V_{3}$ of $V_{2}$ such that $\operatorname{rad}\left(V_{3}\right)=0$ and $\operatorname{rad}\left(V_{0}\right) \leqslant V_{3}$. If $V$ is an orthogonal space with $q$ even and if $\operatorname{rad}\left(V_{0}\right)$ is nonsingular, then choose $V_{3}$ so that $V_{3}$ contains no singular 1-spaces. Then $I \geqslant A \times 9\left(V_{3}\right)$ and we will show that $\varphi\left(V_{1} \perp V_{3}\right) \leqslant I$. To see this let $P$ be the stabilizer in $G\left(V_{1} \perp V_{3}\right)$ of $\operatorname{rad}\left(V_{0}\right)$ and $P_{0}=C_{P}\left(\operatorname{rad}\left(V_{0}\right)\right)$. For the moment exclude the case where $V$ is orthogonal and $q$ is even. Then $P_{0}=O_{r}(P) O^{r^{\prime}}(A)$ and acts irreducibly on $O_{r}(P) / O_{r}\left(Z\left(P_{0}\right)\right.$ ) (see $\S 3$ of [10]). Since $c \in N\left(P_{0}\right)$ but $c \notin N(A)$ we have $\langle A, c\rangle$ covering $O_{r}(P) \bmod O_{r}\left(Z\left(P_{0}\right)\right)$. The results in $\S 3$ of [10] imply that $O_{r}\left(Z\left(P_{0}\right)\right)=\Phi\left(O_{r}(P)\right)$, unless $X$ is a symplectic group in characteristic 2 . In the latter case $O_{r}(P)$ is indecomposable as an $A$-module, unless $X \cong S p(4,2)$, where the result is easy to check. So we may now assume that $\langle A, c\rangle \geqslant P_{0}$, and so $P_{0} \leqslant I$. Also $I \geqslant C_{X}\left(V_{1}\right) \geqslant C_{X}\left(V_{1}\right) \cap C_{X}\left(V_{3}^{\perp}\right)$ and $P_{0}$ together with this last group generates a group containing $G\left(V_{1} \perp V_{3}\right)$. To complete this case consider the decomposition $V_{1} \perp V_{3} \perp\left(V_{3}^{\perp} \cap V_{2}\right)$ and apply (3.1) to conclude that $9(V) \leqslant I$. So $X=I$.

Now suppose that $V$ is orthogonal and $q$ is even. If $\operatorname{rad}\left(V_{0}\right)$ is singular the same arguments work, once we note that $I$ contains a transvection that stabilizes $V_{3}$ but not $\operatorname{rad}\left(V_{0}\right)$. So suppose $\operatorname{rad}\left(V_{0}\right)$ is nonsingular. Then $\langle A, c\rangle \leqslant P_{0}=C\left(\operatorname{rad}\left(V_{0}\right)\right) \cap 9\left(V_{1} \perp V_{3}\right) \cong S p(k, q)$, where $k=\operatorname{dim}\left(V_{1}\right)$. We note that $\langle A, c\rangle=P_{0}$. To see this note that $V_{1} \cap V_{1}^{c}$ is a hyperplane in $V_{1}$, so $\left|A \cap A^{c}\right|$ can be computed. Then count the number of elements in $A A^{c}$ and conclude from [17]

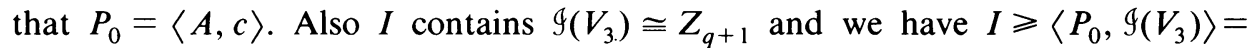
$g\left(V_{1} \perp V_{3}\right)$. At this point we can choose a transvection $c^{\prime} \in I$ such that $c^{\prime} \notin N(A)$ and $\operatorname{rad}\left(V_{0}^{\prime}\right)$ is singular, where $V_{0}^{\prime}=\left[\left\langle A, c^{\prime}\right\rangle, V\right]$. Namely, first write $V=$ $V_{1} \perp V_{3} \perp V_{4}$, where $V_{4}=V_{2} \cap V_{3}^{\perp}$. Let $c_{1}$ be a transvection in $C\left(V_{1}\right)$ with $\left[V, c_{1}\right]=$ $v_{3}+v_{4}$, where $v_{3} \in V_{3}, v_{4} \in V_{4}$, and $0 \neq v_{4}$ is singular. Let $g \in \mathscr{G}\left(V_{1} \perp V_{3}\right)$ with $v_{3}^{g} \in V_{1}$. Then $c^{\prime}=c_{1}^{g}$ is such a transvection. Now argue as above to get the result.

Next we suppose $\operatorname{rad}\left(V_{0}\right)=0$. Then $X$ is neither a symplectic space nor an orthogonal space with $q$ even. If $V$ is an orthogonal space with $q=3$, let $U$ be a nondegenerate 3-space of $V_{2}$ with $U \geqslant V_{0} \cap V_{2}$. Otherwise let $U$ be a nondegenerate 2-space of $V_{2}$ with $U \geqslant V_{0} \cap V_{2}$. Unless $V$ is orthogonal and $\operatorname{dim}\left(V_{2}\right)=2$ we may 
assume that $U$ contains an isotropic 1-space. Inductively we may assume $U=V_{2}$. Indeed, write $V=V_{1} \perp U \perp\left(V_{2} \cap U^{\perp}\right)$ and apply (3.1).

Next we write $V_{1}=V_{1}^{\prime} \perp V_{1}^{\prime \prime}$, where $V_{1}^{\prime \prime}$ has dimension at least 2 if $V$ is a unitary space and at least 4 if $V$ is an orthogonal space. Further, choose $V_{1}^{\prime \prime}$ so that $V_{1}^{\prime \prime} \perp V_{2} \geqslant[V, c]$. Now consider the decomposition $V=V_{1}^{\prime} \perp V_{1}^{\prime \prime} \perp V_{2}$ and apply induction together with (3.1). We conclude that one of the following occurs: $I=X$, $V_{1}^{\prime}=0$, or $X=S U(n, 2)$ and $V_{1}^{\prime \prime}$ is a 2 -space. In the latter case, we can replace $V_{1}^{\prime \prime}$ by a proper nondegenerate 3 -space of $V_{1}$ containing $V_{1}^{\prime \prime}$, if $n \geqslant 6$. Here, (3.1) and induction yield $I=X$. So from now on we may assume that either $X \cong S U(5,2)$ or $V_{1}^{\prime}=0$ and $X \cong S U(4, q), q>2, O^{ \pm}(6, q), q>3$, or $O^{ \pm}(7,3)$.

Suppose $X \cong S U(5,2)$. Then $A \cong S U(3,2)$. The space $V_{1} \cap V_{1}^{c}$ is $c$-invariant, while $[V, c] \neq V_{1}$. It follows that $V_{1} \cap V_{1}^{c}$ is a 2-space in $C_{V_{0}}(c)$, hence nondegenerate. As $A \cap A^{c}$ stabilizes $V_{1} \cap V_{1}^{c}, A \cap A^{c} \cong S_{3} \times Z_{3}$. Thus, $\left\langle A, A^{c}\right\rangle \geqslant\left|A A^{c}\right| \geqslant 12|A|$. We claim that $\langle A, c\rangle=S U(4,2)$. As $\langle A, c\rangle \leqslant \varphi\left(V_{0}\right),\langle A, c\rangle \leqslant S U(4,2)$. Elementary order considerations show that either the claim holds or $\langle A, c\rangle$ has index 10 in $9\left(V_{0}\right) \cong P S p(4,3)$, so in the latter case $\langle A, c\rangle$ would be contained in a proper parabolic subgroup of $\operatorname{PSp}(4,3)$. Checking orders we have a contradiction. Therefore, $\mathscr{Q}\left(V_{0}\right) \leqslant I$. Choose $g \in \mathscr{T}\left(V_{2}\right)$ such that $V=V_{0}+V_{0}^{g}$. Then $\mathscr{}\left(V_{0}^{g}\right) \leqslant I$ and by (3.1) $I \geqslant\left\langle 9\left(V_{0}\right), \mathscr{}\left(V_{0}^{g}\right)\right\rangle=9(V)$. From now on we assume $V_{1}^{\prime}=0$.

Suppose $X \cong S U(4, q)$ with $q>2$. As above it will suffice to show that $q\left(V_{0}\right) \leqslant I$. But this follows from a check of subgroups of $S U(3, q)$ (see [5] and [14]). Now suppose $X \cong O^{ \pm}(6, q)$ with $q>3$ or $X \cong O^{ \pm}(7,3)$. Here $9\left(V_{0}\right) \cong P \operatorname{Sp}(4, q)$, $V_{0}=\left\langle V_{1}, V_{1}^{c}\right\rangle$, and $V_{1} \cap V_{1}^{c}$ is a 3-space stabilized by the involution $c$. Set $L=\langle A, c\rangle$, so that $|L| \geqslant\left|A A^{c}\right|=|A| \cdot\left|A: A \cap A^{c}\right|$. Computing the possible choices for $A \cap A^{c}$ (which stabilizes the hyperplane $V_{1} \cap V_{1}^{c}$ of $V_{1}$ ), we have $\left|\mathscr{G}\left(V_{0}\right)\langle c\rangle: L\right| \leqslant \frac{1}{2} q^{2}$. Apply the theorem of [17] to conclude that $L=\mathscr{G}\left(V_{0}\right)\langle c\rangle$.

If $X=O^{ \pm}(6, q)$ with $q>3$, then choose $g \in \mathscr{q}(U)$ such that $V_{0} \cap U \neq V_{0}^{g} \cap U$. Then $I \geqslant\left\langle 9\left(V_{0}\right), g, c\right\rangle$, and arguing as above we have $I=X$. This leaves the case $X \cong O^{ \pm}(7,3)$. Let $\left\langle v_{0}\right\rangle=V_{0} \cap U$ and choose $g \in \mathscr{G}(U)$ such that $\left\langle v_{0}, v_{0}^{g}\right\rangle$ is nondegenerate but contains isotropic vectors. Then (3.1) and induction yield $9\left(\left\langle V_{1}, v_{0}, v_{0}^{g}\right\rangle\right) \leqslant I$. A further application of (3.1) shows that $X=I$.

Let $G_{0}=G_{0}(q)$ be an exceptional Chevalley group (notation as in \$2). We will produce certain subgroups $L$ of $G_{0}$. These subgroups are described below in Table (3.3). Here $L_{0}=O^{r^{\prime}}(L), C=C_{L}\left(L_{0}\right)$, and $F=L / L_{0} C$. In all cases each component, $X$, of $L_{0}$ is normal in $L$, and $L / C_{L}(X)$ induces inner and diagonal automorphisms of $X$.

To prove the existence of $L$ we consider $G_{0}=\hat{G}_{0}$ and argue as follows. We choose a certain $\sigma$-invariant subgroup $\hat{L}$ of $\hat{G}$, which usually is generated by root subgroups of $\hat{G}$. Then we produce an element $\hat{w}$ of the Weyl group of $\hat{G}$ such that $\hat{w}$ stabilizes $\hat{L}$. By Lang's theorem $([18]$ or $(10.1)$ of $[23]) \hat{w} \sigma$ and $\sigma$ are conjugate in $\hat{G}$. Consequently $G_{0}$ contains a $\hat{G}$-conjugate of $\hat{L}_{w \sigma}$, and this is the appropriate subgroup $L$. Recall that root diagrams are labeled as in Table (2.17).

Choose a $\sigma$-invariant Borel subgroup, $\hat{B}$, of $\hat{G}$ so that the $(B, N)$-structure of $G_{0}$ is obtained in the usual way from that of $\hat{G}$. So $H_{0}=\hat{T}_{\sigma}$, for $\hat{T}=\hat{T}^{\sigma}$ a maximal torus in $\hat{B}$, and $\sigma$ acts on the root subgroups of $\hat{B}_{u}=U$ in the usual way. 
TABLE (3.3)

\begin{tabular}{|c|c|c|c|c|}
\hline$G_{0}$ & $L_{0} / Z\left(L_{0}\right)$ & C & $Z\left(L_{0}\right)$ & $F$ \\
\hline$E_{6}(q)$ & $\begin{array}{l}L_{3}(q) \times L_{3}(q) \times L_{3}(q) \\
L_{3}\left(q^{2}\right) \times U_{3}(q)\end{array}$ & $\begin{array}{l}Z_{(3, q-1)} \times Z_{(3, q-1)} \\
Z_{\left(3, q^{2}-1\right)}\end{array}$ & $\begin{array}{l}Z_{(3, q-1)} \times Z_{(3, q-1)} \\
Z_{\left(3, q^{2}-1\right)}\end{array}$ & $\begin{array}{l}Z_{(3, q-1)} \\
Z_{\left(3, q^{2}-1\right)}\end{array}$ \\
\hline$E_{7}(q)$ & $\begin{array}{l}{ }^{3} D_{4}(q) \times L_{2}\left(q^{3}\right) \\
{ }^{2} E_{6}(q) \\
U_{3}(q) \times U_{6}(q)\end{array}$ & $\begin{array}{l}Z_{(2, q-1)} \\
Z_{q+1} \\
Z_{(3, q+1)}\end{array}$ & $\begin{array}{l}Z_{(2, q-1)} \\
Z_{(3, q+1)} \\
Z_{(3, q+1)}\end{array}$ & $\begin{array}{l}Z_{(2, q-1)} \\
Z_{(3, q+1)} \\
Z_{(3, q+1)}\end{array}$ \\
\hline$E_{8}(q)$ & $\begin{array}{l}D_{4}(q) \times D_{4}(q) \\
{ }^{3} D_{4}(q) \times{ }^{3} D_{4}(q) \\
L_{5}(q) \times L_{5}(q) \\
U_{5}(q) \times U_{5}(q) \\
L_{3}(q) \times E_{6}(q) \\
U_{3}(q) \times{ }^{2} E_{6}(q) \\
P S O^{-}(12, q)^{\prime} \times L_{2}\left(q^{2}\right) \\
L_{9}(q)\end{array}$ & $\begin{array}{l}Z_{(2, q-1)} \times Z_{(2, q-1)} \\
1 \\
Z_{(5, q-1)} \\
Z_{(5, q+1)} \\
Z_{(3, q-1)} \\
Z_{(3, q+1)} \\
Z_{(2, q-1)} \\
Z_{(3, q-1)}\end{array}$ & $\begin{array}{l}Z_{(2, q-1)} \times Z_{(2, q-1)} \\
1 \\
Z_{(5, q-1)} \\
Z_{(5, q+1)} \\
Z_{(3, q-1)} \\
Z_{(3, q+1)} \\
Z_{(2, q-1)} \\
Z_{n}, \\
n=\frac{(9, q-1)}{(3, q-1)}\end{array}$ & $\begin{array}{l}Z_{(2, q-1)} \times Z_{(2, q-1)} \\
1 \\
Z_{(5, q-1)} \\
Z_{(\zeta, q+1)} \\
Z_{(3, q-1)} \\
Z_{(3, q+1)} \\
Z_{(2, q-1)} \\
Z_{n}, \\
n=\frac{(9, q-1)}{(3, q-1)}\end{array}$ \\
\hline & $U_{9}(q)$ & $Z_{(3, q+1)}$ & $\begin{array}{l}Z_{n}, \\
n=\frac{(9, q+1)}{(3, q+1)}\end{array}$ & $\begin{array}{l}Z_{n}, \\
n=\frac{(9, q+1)}{(3, q+1)}\end{array}$ \\
\hline${ }^{2} E_{6}(q)$ & $U_{3}(q) \times U_{3}(q) \times U_{3}(q)$ & $Z_{(3, q+1)} \times Z_{(3, q+1)}$ & $Z_{(3, q+1)} \times Z_{(3, q+1)}$ & $Z_{(3, q+1)}$ \\
\hline$F_{4}(q)$ & $\begin{array}{l}L_{3}(q) \times L_{3}(q) \\
U_{3}(q) \times U_{3}(q)\end{array}$ & $\begin{array}{l}Z_{(3, q-1)} \\
Z_{(3, q+1)}\end{array}$ & $\begin{array}{l}Z_{(3, q-1)} \\
Z_{(3, q+1)}\end{array}$ & $\begin{array}{l}Z_{(3, q-1)} \\
Z_{(3, q+1)}\end{array}$ \\
\hline$G_{2}(q)$ & $\begin{array}{l}L_{3}(q) \\
U_{3}(q)\end{array}$ & $\begin{array}{l}Z_{(3, q-1)} \\
Z_{(3, q+1)}\end{array}$ & $\begin{array}{l}Z_{(3, q-1)} \\
Z_{(3, q+1)}\end{array}$ & $\begin{array}{l}1 \\
1\end{array}$ \\
\hline$D_{4}(q)$ & $\begin{array}{l}L_{3}(q) \\
U_{3}(q)\end{array}$ & $\begin{array}{l}Z_{q-1} \times Z_{q-1} \\
Z_{q+1} \times Z_{q+1}\end{array}$ & $\begin{array}{l}Z_{(3, q-1)} \\
Z_{(3, q+1)}\end{array}$ & $\begin{array}{l}Z_{(3, q-1)} \\
Z_{(3, q+1)}\end{array}$ \\
\hline${ }^{3} D_{4}(q)$ & $\begin{array}{l}L_{3}(q) \\
U_{3}(q)\end{array}$ & $\begin{array}{l}Z_{\left(q^{2}+q+1\right)} \\
Z_{\left(q^{2}-q+1\right)}\end{array}$ & $\begin{array}{l}Z_{(3, q-1)} \\
Z_{(3, q+1)}\end{array}$ & $\begin{array}{l}Z_{(3, q-1)} \\
Z_{(3, q+1)}\end{array}$ \\
\hline
\end{tabular}

Let $\pi=\left\{\alpha_{1}, \ldots, \alpha_{n}\right\}$ be a fundamental system of roots for $W$. For the cases $E_{6}$, $E_{7}, E_{8}$ we label the Dynkin diagram as follows:

$$
\begin{array}{ccc}
\alpha_{1} \alpha_{3} \alpha_{4} \alpha_{5} \alpha_{6} & \alpha_{1} \alpha_{3} \alpha_{4} \alpha_{5} \alpha_{6} \alpha_{7} & \alpha_{1} \alpha_{3} \alpha_{4} \alpha_{5} \alpha_{6} \alpha_{7} \alpha_{8} \\
\alpha_{2} & \alpha_{2} & \alpha_{2}
\end{array}
$$

In all cases $-s$ will denote the positive root of highest height (with respect to $\pi$ ). Listed below are various other fundamental systems for $E_{6}, E_{7}, E_{8}$ :

$$
\begin{aligned}
& E_{6} \quad \pi_{1} \quad \alpha_{5} \alpha_{6} t \alpha_{1} \alpha_{3}, \quad \text { where } t=\alpha_{2}+\alpha_{3}+2 \alpha_{4}+\alpha_{5} \\
& s \\
& E_{7} \quad \pi_{2} \alpha_{6} \alpha_{5} \alpha_{4} \alpha_{3} \alpha_{1} s \\
& \alpha_{2} \\
& \pi_{3} \text { st } \alpha_{6} \alpha_{5} \alpha_{4} \alpha_{2}, \quad \text { where } t=\alpha_{1}+\alpha_{2}+2 \alpha_{3}+2 \alpha_{4}+\alpha_{5} \\
& \alpha_{7} \\
& E_{8} \quad \pi_{4} \quad \alpha_{6} \alpha_{5} \alpha_{4} \alpha_{2} t_{1} t_{2} \alpha_{8} \text {, where } t_{1}=-\left(\alpha_{1}+2 \alpha_{2}+2 \alpha_{3}+3 \alpha_{4}+2 \alpha_{5}+\alpha_{6}\right) \\
& \alpha_{3} \quad \text { and } t_{2}=2 \alpha_{1}+2 \alpha_{2}+3 \alpha_{3}+4 \alpha_{4}+3 \alpha_{5}+2 \alpha_{6}+\alpha_{7} \\
& \pi_{5} \alpha_{2} \alpha_{4} \alpha_{3} t s \alpha_{8} \alpha_{7}, \quad \text { where } t=\alpha_{1}+2 \alpha_{2}+2 \alpha_{3}+4 \alpha_{4} \\
& \alpha_{1} \quad+4 \alpha_{5}+3 \alpha_{6}+2 \alpha_{7}+\alpha_{8} \\
& \pi_{6} \quad \alpha_{6} \alpha_{5} \alpha_{4} \alpha_{3} \alpha_{1} t s, \quad \text { where } t=\alpha_{2}+\alpha_{3}+2 \alpha_{4}+2 \alpha_{5}+2 \alpha_{6}+2 \alpha_{7}+\alpha_{8} \\
& \alpha_{2}
\end{aligned}
$$




$$
\begin{array}{ll}
\pi_{7} s \alpha_{8} \alpha_{7} \alpha_{6} \alpha_{5} \alpha_{4} \alpha_{3}, & \text { where } t=\alpha_{1}+2 \alpha_{2}+2 \alpha_{3}+3 \alpha_{4}+2 \alpha_{5}+\alpha_{6} \\
t & \\
\pi_{8} t_{1} \alpha_{2} \alpha_{4} \alpha_{5} \alpha_{6} \alpha_{7} t_{2}, & \text { where } t_{1}=\alpha_{1}+\alpha_{3}+\cdots+\alpha_{8}, \text { and } \\
\alpha_{3} & t_{2}=-\left(\alpha_{2}+\alpha_{3}+2 \alpha_{4}+2 \alpha_{5}+2 \alpha_{6}+2 \alpha_{7}+\alpha_{8}\right)
\end{array}
$$

Since the Weyl group is transitive on the set of all fundamental systems of roots, we may choose $w_{i} \in W$ such that $\pi^{w_{i}}=\pi_{i}, i=1, \ldots, 8$. For $i>1, w_{i}$ necessarily preserves the labeling of the Dynkin diagram. Setting $w_{1}=\left(s_{4} s_{1} s_{6}\right)^{s_{3} s_{5} s_{4} s_{2}}$, we also have this for $i=1$.

Let $G_{0}=E_{6}(q)$. Then $\hat{G}=E_{6}(K)$ and $\sigma$ is a field automorphism. Let $X_{1}=\left\langle\hat{U}_{ \pm \alpha_{1}}, \hat{U}_{ \pm \alpha_{3}}\right\rangle, \quad X_{2}=\left\langle\hat{U}_{ \pm \alpha_{5}}, \hat{U}_{ \pm \alpha_{6}}\right\rangle, \quad$ and $X_{3}=\left\langle\hat{U}_{ \pm \alpha_{2}}, \hat{U}_{ \pm s}\right\rangle$. Then $X_{1} \cong X_{2} \cong X_{3} \cong S L(3, K)$ and $\left[X_{1}, X_{2}\right]=\left[X_{2}, X_{3}\right]=\left[X_{1}, X_{3}\right]=1$. We note that $X_{1} X_{2}=X_{1} \times X_{2}$. This can be seen by viewing $E_{6}(K) \leqslant E_{7}(K)$, then considering $X_{1} X_{2}$ as a subgroup of $S L(7, K) \leqslant E_{7}(K)$. Conjugation within the Weyl group of $G_{0}$ yields $X_{2} X_{3} \sim X_{1} X_{3} \sim X_{1} X_{2} \cong X_{1} \times X_{2}$. On the other hand, we claim that $Z\left(X_{1} X_{2} X_{3}\right) \cong Z_{3} \times Z_{3}$ if $r \neq 3$. From the above we see that either this holds or $Z=Z\left(X_{1} X_{2} X_{3}\right) \cong Z_{3} \times Z_{3} \times Z_{3}$. If the latter holds consider $C_{Z}\left(\hat{U}_{\alpha_{4}}\right)=Z_{0}$. Then $Z_{0}$ also centralizes $\hat{U}_{-\alpha_{4}}$; hence $Z_{0} \leqslant C\left(\left\langle U_{ \pm \alpha_{i}} \mid i=1, \ldots, 6\right\rangle\right)=Z(\hat{G})$. However, $\left|Z_{0}\right| \geqslant 9$, whereas $|Z(\hat{G})|=3$. This proves the claim and gives the group $L_{0}$ for the first case in $E_{6}(q)$. Namely, set $L_{0}=\left(X_{1}\right)_{\sigma}\left(X_{2}\right)_{\sigma}\left(X_{3}\right)_{\sigma}$. Let $\left\langle z_{i}\right\rangle=Z\left(X_{i}\right)$ for $i=1,2,3$. Notation may be chosen such that $z_{1} z_{2} z_{3}=1$. For each $i=1,2,3$ we apply Lang's theorem $\left((10.1)\right.$ of [23]) to obtain an element $a_{i} \in X_{i}$ such that $a_{i}^{\sigma}=a_{i} z_{i}$. Then $a^{\sigma}=a$, where $a=a_{1} a_{2} a_{3}$. Also $a \in N\left(L_{0}\right)$ (each $a_{i} \in N\left(\left(X_{i}\right)_{\sigma}\right)$ ) and $a \notin L_{0} Z\left(X_{1} X_{2} X_{3}\right)$ precisely if $3 \mid q-1$. Let $L=L_{0}\langle a\rangle, L_{0}$, or $L_{0}$ according to $q \equiv 1,-1$, or $0(\bmod 3)$. Then $L$ is the first of the groups for $E_{6}(q)$.

The element $w_{1} \in W$ is an involution and it is clear that $X_{1}^{w_{1}}=X_{2}$, while $X_{3}^{w_{1}}=X_{3}$, and $w_{1}$ induces a graph automorphism on the Dynkin diagram of $X_{3}$. Let $g=w_{1} \sigma$. Then $g \sim \sigma$ by Lang's theorem ((10.1) of [23]). We may take a coset representative of $w_{1}$ in $\left(G_{0}\right)_{\sigma}$, so $g^{2} \in \sigma^{2} H$ and another application of Lang's theorem yields $\left(X_{i}\right)_{g^{2}} \cong S L\left(3, q^{2}\right)$ for $i=1,2,3$. Therefore, $\left(X_{1} X_{2}\right)_{g} \cong S L\left(3, q^{2}\right)$. From the action of $g$ on $X_{3}$ we have $\left(X_{3}\right)_{g} \cong S U(3, q)$ (see [23, (11.2) and (11.6)]). Let $L_{0}=\left(X_{1} X_{2}\right)_{g}\left(X_{3}\right)_{g}$. Since $X_{1}^{g}=X_{2}$ we have $Z\left(L_{0}\right) \cong Z_{\left(3, q^{2}-1\right)}$. If $\left(3, q^{2}-1\right)=1$, set $L=L_{0}$, and if $3 \mid q^{2}-1$ set $L=L_{0}\langle x y\rangle$, where $x \in X_{1} X_{2}$, $y \in X_{3}, x^{g}=x z_{1} z_{2}$, and $y^{g}=y z_{3}$. Then $L \leqslant \hat{G}_{g} \cong E_{6}(q)$ and we have the other subgroup for $E_{6}(q)$.

The above set-up can be used to describe the required subgroups for $F_{4}(q)$ and ${ }^{2} E_{6}(q)$. Let $\tau$ be the graph automorphism of $\hat{G}$, defined with respect to $\hat{B}, \hat{H}, \hat{N}$, etc. Let $X=X_{1} X_{2} X_{3}$ and note that we may take $w \in \hat{G}_{\tau} \cong F_{4}(K)$. Consequently, $F_{4}(q)=\left(\hat{G}_{\tau}\right)_{\sigma}$ contains conjugates of $X_{\tau} \cap X_{g}$ and $X_{\tau} \cap X_{\sigma}$. Now $\tau$ centralizes $X_{3}$, so $z_{3}^{\tau}=z_{3}$. As $1=\left(z_{1} z_{2} z_{3}\right)^{\tau}=z_{1} z_{2} z_{3}$ we must have $z_{1}^{\tau}=z_{2}$ and $z_{2}^{\tau}=z_{1}$. So $Z(X)_{\tau}=$ $\left\langle z_{3}\right\rangle$. Since $\left[X_{3}, \tau\right]=1,\left(X_{3}\right)_{g}$ or $\left(X_{3}\right)_{\sigma}$ is contained in $X_{\tau} \cap X_{g}$ or $X_{\tau} \cap X_{\sigma}$, respectively. Also $\left(\left(X_{1} X_{2}\right)_{\tau}\right)_{\sigma} \cong S L(3, q)$ and $\left(\left(X_{1} X_{2}\right)_{\tau}\right)_{g} \cong S U(3, q)$. This gives the subgroups $L_{0}$ for $F_{4}(q)$ and the usual argument gives $L=L_{0}$ or $L_{0}\langle a\rangle$, completing the case of $F_{4}(q)$. For $G_{0}={ }^{2} E_{6}(q)$ note that $\tau \sigma \sim w_{1} \tau \sigma$ so $G_{0}$ contains a conjugate of 
$X_{w_{1} \tau \sigma}$. The element $w_{1} \tau \sigma$ stabilizes each $X_{i}$ and $\left(X_{i}\right)_{w_{1} \tau \sigma} \cong S U(3, q)$. From here the usual argument shows that $L=X_{w \tau \sigma}$ is the required subgroup.

Next, let $G_{0}=E_{8}(q)$ and $\hat{G}=E_{8}(K)$. We produce central products $\hat{X}=\hat{X}_{1} \hat{X}_{2}$ and $\hat{Y}=\hat{Y}_{1} \hat{Y}_{2}$ as follows. Let

$$
\hat{X}_{1}=\left\langle\hat{U}_{ \pm \alpha_{2}}, \hat{U}_{ \pm \alpha_{3}}, \hat{U}_{ \pm \alpha_{4}}, \hat{U}_{ \pm \alpha_{5}}\right\rangle
$$

and

$$
\hat{X}_{2}=\left\langle\hat{U}_{ \pm \alpha_{7}}, \hat{U}_{ \pm \alpha_{8}}, \hat{U}_{ \pm t_{1}}, \hat{U}_{ \pm t_{2}}\right\rangle \text {, }
$$

where $t_{1}=2 \alpha_{1}+2 \alpha_{2}+3 \alpha_{3}+4 \alpha_{4}+3 \alpha_{5}+2 \alpha_{6}+\alpha_{7}$ and $t_{2}=\alpha_{2}+\alpha_{3}+2 \alpha_{4}+$ $2 \alpha_{5}+2 \alpha_{6}+\alpha_{7}$. Let $\hat{Y}_{1}=\left\langle\hat{U}_{ \pm \alpha_{i}} \mid 1 \leqslant i \leqslant 4\right\rangle$ and $\hat{Y}_{2}=\left\langle\hat{U}_{ \pm \alpha_{6}}, \hat{U}_{ \pm \alpha_{7}}, \hat{U}_{ \pm \alpha_{8}}, U_{ \pm s}\right\rangle$.

From the commutator relations we have $\left[\hat{X}_{1}, \hat{X}_{2}\right]=\left[\hat{Y}_{1}, \hat{Y}_{2}\right]=1$. Each of $\hat{X}_{1}$ and $\hat{X}_{2}$ is a perfect central extension of $D_{4}(K)$ and each of $\hat{Y}_{1}$ and $\hat{Y}_{2}$ is a perfect central extension of $L_{5}(q)$. An easy computation shows that $Z\left(X_{1}\right)=Z\left(X_{2}\right)=1$ or $Z_{2} \times Z_{2}$, according to whether or not $r=5$. Similarly, $Z\left(Y_{1}\right)=Z\left(Y_{2}\right) \cong 1$ or $Z_{5}$, according to whether or not $r=2$. From the action of $\sigma$ on $X=\hat{X}_{1} \hat{X}_{2}$ and on $Y=\hat{Y}_{1} \hat{Y}_{2}$ we obtain the required subgroup $L\left(L=X_{\sigma}\right.$ or $\left.Y_{\sigma}\right)$ with $L_{0} / Z\left(L_{0}\right) \cong D_{4}(q) \times D_{4}(q)$ or $L_{5}(q) \times L_{5}(q)$.

From the action of $w_{4}$ and $w_{5}$ on $\pi$ we see that $X_{i}^{w_{4}}=X_{i}$ for $i=1,2$ and $Y_{i}^{w_{5}}=Y_{i}$ for $i=1,2$. Moreover, $w_{4}$ induces a triality graph automorphism on the Dynkin diagram of $X_{1}$ and $X_{2}$, while $w_{5}$ induces the involutory graph automorphism on the Dynkin diagram of $Y_{1}$ and $Y_{2}$. So considering $w_{4} \sigma$ and $w_{5} \sigma$ we obtain the groups $L$ with $L_{0} / Z\left(L_{0}\right) \cong{ }^{3} D_{4}(q) \times{ }^{3} D_{4}(q)$ and $U_{5}(q) \times U_{5}(q)$. We can also restrict to $E_{7}(K) \leqslant E_{8}(K)$ and obtain the first assertion for $E_{7}(q)$. Namely, look at $X_{1} \times X_{3}$, with $X_{3}=\left\langle\hat{U}_{ \pm \alpha_{7}}\right\rangle \times\left\langle\hat{U}_{ \pm t_{1}}\right\rangle \times\left\langle\hat{U}_{ \pm t_{2}}\right\rangle$, use the element $w_{4} \sigma$, and argue as usual.

Next, set $Z_{1}=\left\langle\hat{U}_{ \pm \alpha_{1}}, \ldots, \hat{U}_{ \pm \alpha_{6}}\right\rangle$ and $Z_{2}=\left\langle\hat{U}_{ \pm \alpha_{8}}, \hat{U}_{ \pm s}\right\rangle$. Then $\left[Z_{1}, Z_{2}\right]=1, Z_{1}$ is a perfect central extension of $E_{6}(K)$ (in fact the universal group) and $Z_{2} \cong S L(3, K)$. The argument used for the case $E_{6}(K)$ and the fact that $3+|Z(\hat{G})|$ imply that $Z\left(Z_{1}\right)=Z\left(Z_{2}\right)$. Letting $L_{0}=\left(Z_{1}\right)_{\sigma}\left(Z_{2}\right)_{\sigma}$ we have $L_{0} / Z\left(L_{0}\right) \cong E_{6}(q) \times L_{3}(q)$ and $Z\left(L_{0}\right) \cong Z_{(3, q-1)}$. Fix $z \in Z\left(Z_{1} Z_{2}\right)^{\#}$ and choose $a \in Z_{1}$ and $b \in Z_{2}$ with $a^{\sigma}=a z$ and $b^{\sigma}=b z^{-1}$. This is possible by Lang's theorem. Then $\sigma$ centralizes $a b$ and $L=L_{0}\langle a b\rangle$ satisfies the conditions for the appropriate subgroup of $E_{8}(q)$.

Let $g=w_{6} \sigma$. The usual arguments show that $L=\left(Z_{1} Z_{2}\right)_{g}$ is the twisted version of the previous example. Namely $O^{r^{\prime}}(L)=L_{0}$ satisfies $L_{0} / Z\left(L_{0}\right) \cong{ }^{2} E_{6}(q) \times U_{3}(q)$. A variation of this example leads to the second group for $E_{7}(q)$. Namely, use $g=w_{2} \sigma$ (acting on $\left.E_{7}(K)\right)$. As $w_{2}$ induces an involutory graph automorphism of $Z_{1}$, we see that $\left(Z_{1}\right)_{g}$ is the covering group of ${ }^{2} E_{6}(q)$. Also, if we set $T=\left\{h(\chi) \mid \chi\left(\alpha_{i}\right)=1\right.$ for $i \neq 7\}$, then $\left[Z_{1}, T\right]=1$ and $T$ is a 1 -dimensional torus. Now $Z\left(Z_{1}\right) \leqslant T$ and since $\alpha_{7}^{\omega_{2}}=-s, g$ inverts $T$. Thus, $T_{g} \cong Z_{q+1}$ and $L=\left(Z_{1} T\right)_{g}$ is the desired group.

To complete $E_{7}(q)$, let $V_{1}=\left\langle\hat{U}_{ \pm \alpha_{2}}, \hat{U}_{ \pm \alpha_{4}}, \ldots, \hat{U}_{ \pm \alpha_{7}}\right\rangle$ and $V_{2}=\left\langle\hat{U}_{ \pm \alpha_{1}}, \hat{U}_{ \pm s}\right\rangle$. Argue as usual to get $L=\left(V_{1} V_{2}\right)_{w_{3} \sigma}$ as indicated in Table (3.3).

We continue the analysis of $E_{8}(q)$. Set $D=\left\langle\hat{U}_{ \pm \alpha_{1}}, \hat{U}_{ \pm \alpha_{3}}, \ldots, \hat{U}_{ \pm \alpha_{8}}, \hat{U}_{ \pm s}\right\rangle$. Then $D$ is a perfect central extension of $L_{9}(K)$ and checking the action of $\hat{H}$ we have 
$Z(D)=1$ or $Z_{3}$, according to $r=3$ or $r \neq 3$. The covering group of $D$ is $S L(9, K)$. Let $L=D_{\sigma}$ and $L_{0}=O^{r^{\prime}}(L)$. If $r \neq 3$, then (2.13) implies that $\left|L: L_{0}\right|=3$. Also, considering the action of $\sigma$ on $S L(9, K)$ we see that $3 \| q-1$, then $L=L_{0} \times Z_{3}$. So $L$ satisfies the conditions in Table (3.3). To produce the twisted version of this example let $g=w_{7} \sigma$ and argue in the usual manner.

To complete the analysis of $E_{8}(q)$ we must produce a central extension of $P S O^{-}(12, q)^{\prime} \times L_{2}\left(q^{2}\right)$. Let $J_{1}=\left\langle\hat{U}_{ \pm \alpha_{2}}, \ldots, \hat{U}_{ \pm \alpha_{7}}\right\rangle$ and $J_{2}=\left\langle\hat{U}_{ \pm s}, U_{ \pm t}\right\rangle$, where $t$ is the root of highest height in the root system spanned by $\left\{\alpha_{1}, \ldots, \alpha_{7}\right\}$. Then $J_{1} \cong D_{6}(K), J_{2}=\left\langle\hat{U}_{ \pm s}\right\rangle \times\left\langle\hat{U}_{ \pm t}\right\rangle, w_{8}$ induces a graph automorphism of $J_{1}$, and $w_{8}$ interchanges the components of $J_{2}$. Therefore, $\left(J_{1} J_{2}\right)_{w_{8} \sigma}$ satisfies the necessary conditions.

For the remaining cases let $\hat{G}=D_{4}(K)$, with Dynkin diagram

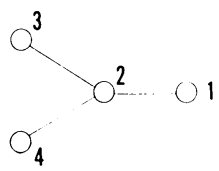

Set $X_{1}=\left\langle\hat{U}_{ \pm \alpha_{2}}, \hat{U}_{ \pm\left(\alpha_{1}+\alpha_{2}+\alpha_{3}+\alpha_{4}\right)}\right\rangle$, so that $X_{1} \cong S L(3, K)$. A direct check shows that $X_{2}=C_{\hat{H}}\left(X_{1}\right)$ is a 2-dimensional torus. Indeed

$$
C_{\hat{H}}\left(X_{1}\right)=\left\{h(\chi): \chi\left(\alpha_{2}\right)=1, \chi\left(\alpha_{1}\right)=\zeta_{1}, \chi\left(\alpha_{3}\right)=\zeta_{2}, \chi\left(\alpha_{4}\right)=\left(\zeta_{1} \zeta_{2}\right)^{-1}\right\} .
$$

As in previous cases we have $Z\left(X_{1}\right) \leqslant X_{2}$ and from here consideration of $L=\left(X_{1} X_{2}\right)_{\sigma}$ gives the first assertion about $D_{4}(q)$. Now let $g=s_{1} s_{2} s_{3}$ and argue with $g \sigma$ to get the second assertion. The results for $G_{2}(q)$ are obtained by observing that the graph automorphism of order 3 of $\hat{G}$ centralizes $X_{1}$. Similarly for ${ }^{3} D_{4}(q)$, but here one must first check the action of $g \sigma$ on $X_{2}$. But this is a straightforward computation. All entries in Table (3.3) have now been accounted for.

For later use we point out that in most of the situations of Table (3.3) the components are generaed by long root subgroups of $G_{0}$. In the notation of Table (3.3) let $L_{0} / Z\left(L_{0}\right)=C_{1} \cdots C_{k}$, where the $C_{i}$ are as indicated in column 2 of Table (3.3). Then each $C_{i}$ is a component of $L_{0} / Z\left(L_{0}\right)$ except when $C_{i} \cong L_{2}(2), L_{2}(3)$, or $U_{3}(2)$. Here we say $C_{i}$ is a solvable component. In the case $U_{2 n+1}(q)$, which has root system of type $B C_{n}$, we will call the long root subgroups those that are nonabelian of order $q^{3}$. We have the following:

(3.4) Let $\left(G_{0}, L_{0}\right)$ be from Table (3.3). Except for the following cases, each $C_{i}$ is generated by the images in $L_{0} / Z\left(L_{0}\right)$ of long root subgroups of $G_{0}$. In each case the indicated component (solvable component) is the only one so generated:

$$
\begin{gathered}
\left(E_{6}(q), L_{3}\left(q^{2}\right) \times U_{3}(q), U_{3}(q)\right), \quad\left(F_{4}(q), U_{3}(q) \times U_{3}(q), U_{3}(q)\right), \\
\left(F_{4}(q), L_{3}(q) \times L_{3}(q), L_{3}(q)\right), \quad\left(E_{7},{ }^{3} D_{4}(q) \times L_{2}\left(q^{3}\right),{ }^{3} D_{4}(q)\right), \\
\left(E_{8}(q), P S O^{-}(12, q)^{\prime} \times L_{2}\left(q^{2}\right), P S O^{-}(12, q)^{\prime}\right) .
\end{gathered}
$$

Proof. In each case $L_{0}=O^{r^{\prime}}(L)$ where $L=\hat{L}_{w \sigma}, \hat{L}$ is a subgroup of $\hat{G}$ generated by certain root subgroups, and $w$ (possibly $w=1$ ) is an element of the Weyl group of $G_{0}$. In each case where $w$ stabilizes a component $\hat{Y}$ of $\hat{L}, w$ also stabilizes a long 
root subgroup of $\hat{L}$ and we easily have $\hat{Y}_{w \sigma}$ generated by long root subgroups. The exceptional cases listed are the ones where $w$ has a nontrivial orbit on the components of $\hat{L}$.

(3.5) Let $\hat{G}=E_{6}(K), \tau$ the field automorphism $k \rightarrow k^{q}$ and $\theta$ the graph automorphism of $\hat{G}$ (defined with respect to $\hat{B}=\hat{B}^{\tau}, \hat{H}=\hat{H}^{\tau}$, etc.).

(i) If $3 \mid q-1$, then $\hat{N}_{\tau}$ contains a Sylow 3-subgroup of $\hat{G}_{\tau}$.

(ii) If $3 \mid q+1$, then $\hat{G}_{\tau \theta}$ contains a subgroup $Y$ with $Y / \operatorname{Fit}(Y) \cong O^{2}(W) \cong$ $O^{-}(6,2)^{\prime}$ and $\operatorname{Fit}(Y)$ is isomorphic to the direct product of 6 copies of $Z_{q+1}$. $Y$ contains a Sylow 3-subgroup of $\hat{G}_{\tau \theta}$.

Proof. The statements concerning Sylow 3-subgroups will follow from the order formulas for $\hat{G}_{\tau}$ and $\hat{G}_{\tau \theta}$, respectively (see (5.1) for example). Now $\tau$ acts on $\hat{N}$ and $\hat{N}=\hat{H}(\hat{N})_{\tau}$. Also $W \cong \hat{W}=\hat{N} / \hat{H} \cong O^{-}(6,2)$. Since $\hat{H}_{\tau}$ is isomorphic to the direct product of 6 copies of $Z_{q-1} \cong \mathbf{F}_{q}^{\#}$ we have (i).

For (ii) let $\tau, \theta$ act on $\hat{N}$. Now $\tau$ centralizes $\hat{N} / \hat{H}$ and $\theta$ induces the graph automorphism on $\hat{N} / \hat{H}$. Let $g \in \hat{N}$ with $g \hat{H}$ the long word in the fundamental reflections generating $\hat{N} / \hat{H}$. Then for each $\alpha \in \hat{\Sigma}, \hat{U}_{\alpha}^{g \theta}=\hat{U}_{-\alpha}$. It follows that $g \theta$ centralizes $\hat{N} / \hat{H}$ and $g \theta$ inverts $\hat{H}$. By Lang's theorem $\tau \theta \sim g \tau \theta$ and by the above $\hat{H}_{g \tau \theta}$ is the direct product of 6 copies of $Z_{q+1}$. So $\hat{G}_{\tau \theta}$ contains a conjugate $Y_{1}$ of $\hat{N}_{g \tau \theta}$ and letting $Y / \operatorname{Fit}\left(Y_{1}\right)=O^{2}\left(Y_{1} / \operatorname{Fit}\left(Y_{1}\right)\right)$ we have the desired group $Y$. (To check that $N_{g \tau \theta}$ covers $\hat{N} / \hat{H}$ use Lang's theorem once again.)

4. Classical groups. In this section we take $G$ to be a classical group. Accordingly, let $G_{0}=S L(V), S p(V), S U(V)$, or $O(V)^{\prime}$, where $V$ is the appropriate module. This is in accordance with the notation in $\$ 2$, except for the orthogonal group in odd characteristic. If $G=P S O^{ \pm}(n, q)^{\prime}$ with $q$ odd, then the group $G_{0}$ of $\S 2$ may be an extension of $S O^{ \pm}(n, q)$ by a group of order 2 . But as $p>2$ this does not affect generation. We omit the case of an orthogonal space of odd dimension and even characteristic, for we identify such groups with symplectic groups.

We must make one additional change of notation regarding the group $E_{0}$. We want $G_{0} E_{0}$ to act on $V$. This certainly happens if $E_{0} \leqslant G_{0}$ (as in the symplectic and orthogonal groups). But for $G_{0}=S L(V)$ or $S U(V)$ this need not occur and we redefine the group $E_{0}$ as follows. Recall that we have $E \leqslant N_{\bar{G}}(G)=P G L(V)$ or $P G U(V)$. Let $E_{0}$ be the Sylow p-subgroup of the preimage in $G L(V)$ or $S U(V)$ of $E$. This change of notation does not affect generation.

Throughout this section we will assume $\operatorname{dim}(V) \geqslant 8$, if $V$ is an orthogonal space.

To describe the cases where generation fails we make the following definition. Suppose $p=3, q=2$, and $G_{0} \not S L(V)$. Let $V=V_{0} \perp V_{1} \perp \cdots \perp V_{k}$ with $V_{0}=C_{V}\left(E_{0}\right)$ and $V_{i}=V_{i}^{E_{0}}$ for $i=1, \ldots, k$. We say this decomposition is admissible if $\operatorname{dim}\left(V_{0}\right) \leqslant 0,1,2$ according to whether $V$ is symplectic, unitary, or orthogonal, and $\operatorname{dim}\left(V_{1}\right)=\cdots=\operatorname{dim}\left(V_{k}\right)=1$ or 2 according to whether $V$ is unitary or not. Note that $k \geqslant 2$.

(4.1) Assume $E_{0}$ is abelian. Then precisely one of the following holds:

(i) $D_{0}=G_{0}$. 
(ii) $p=3, q=2, G_{0} \not S L(V)$ and $V$ contains an admissible decomposition. For any admissible decomposition $V=C_{V}\left(E_{0}\right) \perp V_{1} \perp \cdots \perp V_{k}$, we have $D_{0} \leqslant I$ and $E_{0} \leqslant E_{1}$, where $I$ is the stabilizer in $G_{0} E_{0}$ of $\left\{C_{V}\left(E_{0}\right), V_{1}, \ldots, V_{k}\right\}$ and $E_{1}=O_{3}(I)$.

(iii) $p=3, q=2, G_{0} \cong S p(2 n, 2)$ and $D_{0}=O^{\varepsilon}(2 n, 2)$, where $\varepsilon=(-1)^{n}$. More precisely, $V$ has an admissible decomposition $V=V_{1} \perp \cdots \perp V_{k}$, and for any such decomposition $D_{0}$ preserves the quadratic form which has value 1 on each vector in $V_{i}^{\#}$, for $i=1, \ldots, k$.

In particular, either $D_{0}=G_{0}$ or $E_{0}$ is elementary. Moreover, examples exist for each of (i), (ii) and (iii).

The proof of (4.1) will be carried out in a series of steps. We assume $G_{0}$ to be a counterexample of minimal order. By (2.3) we may assume that $E_{0}$ is contained in no proper parabolic subgroup of $G_{0} E_{0}$. A proper parabolic subgroup of $G_{0} E_{0}$ is the stabilizer of a particular proper subspace, isotropic subspace, or singular subspace of $V$, according to whether $G_{0} \cong S L(V), S p(V)$ or $S U(V)$, or $O^{ \pm}(V)^{\prime}$. So $E_{0}$ stabilizes no such subspace. Since $E_{0}$ is not cyclic $E_{0}$ does not act irreducibly on $V$, and consequently $G_{0} \supsetneqq S L(V)$. We will study $E_{0}$-invariant decompositions of $V$.

Write $V=C_{V}\left(E_{0}\right) \oplus V_{1} \oplus \cdots \oplus V_{k}$, with each $V_{i}$ irreducible $E_{0}$-invariant subspace of $V$. If $W \leqslant V$ with $W \cap W^{\perp}=0$, let $\mathfrak{f}(W)$ be the group generated by all root subgroups of $G_{0}$ contained in $C\left(W^{\perp}\right)$. Except for certain small cases $\dot{j}(W)=\Phi(W)$ as defined in $\S 3$.

(4.2) We may assume that

(i) $V=C_{V}\left(E_{0}\right) \perp V_{1} \perp \cdots \perp V_{k}$.

(ii) $C_{V}\left(E_{0}\right)$ has dimension at most $0,1,2$ depending on whether $G_{0}$ is symplectic, unitary, or orthogonal.

(iii) $\operatorname{dim}\left(V_{1}\right)=\cdots=\operatorname{dim}\left(V_{k}\right)$.

(iv) If $V$ is decomposed as in (i), then $\mathcal{f}\left(C_{V}\left(E_{0}\right) \perp V_{i}\right) \leqslant D_{0}$ for $i=1, \ldots, k$.

Proof. Clearly $V_{1} \oplus \cdots \oplus V_{k}=\left[V, E_{0}\right]$ and so $V_{1} \oplus \cdots \oplus V_{k}=C_{V}\left(E_{0}\right)^{\perp}$. As remarked above, $V_{1}$ is not isotropic (singular if $V$ is an orthogonal space). If $\operatorname{rad}\left(V_{1}\right) \neq 0$ then $G_{0}=S O^{+}(n, q)^{\prime}$ for $q$ a power of 2 , and $V_{1}$ is a 1-space. But here $E_{0}$ must centralize $V_{1}$, whereas $V_{1} \$ C_{V}\left(E_{0}\right)$. So in all cases $\operatorname{rad}\left(V_{1}\right)=0$ and consequently $E_{0}$ acts on $\left(C_{V}\left(E_{0}\right) \perp V_{1}\right)^{\perp}$. If necessary rechoose $V_{2}$ so $V_{2}$ is an irreducible $E_{0}$-invariant subgroup of $\left(C_{V}\left(E_{0}\right) \perp V_{1}\right)^{\perp}$. Continue in this way to get (i).

To get (ii) just notice that $C_{V}\left(E_{0}\right)$ contains no isotropic (singular) 1-space. For $i=1, \ldots, k, E_{0}$ induces a cyclic group of $\operatorname{order} \exp \left(E_{0}\right)$ on $V_{i}$. This determines $\operatorname{dim}\left(V_{i}\right)$ uniquely. So (iii) holds. To see (iv) note that, for $i=1, \ldots, k, E_{0}$ induces a cyclic group of order $\exp \left(E_{0}\right)$ on $C_{V}\left(E_{0}\right) \perp V_{i}$ and that $\Phi\left(E_{0}\right) \leqslant C\left(G_{0}\right) \leqslant$ $C\left(y\left(C_{V}\left(E_{0}\right) \perp V_{i}\right)\right)$. So $\Phi\left(E_{0}\right) C_{E_{0}}\left(C_{V}\left(E_{0}\right) \perp V_{i}\right)$ is a maximal subgroup of $E_{0}$ centralizing $\dot{f}\left(C_{V}\left(E_{0}\right) \perp V_{i}\right)$. The result follows.

(4.3) $k=2$.

Proof. Suppose $k>2$. First assume that either $q>2, p>3$, or $E_{0}$ is not elementary. In each of these cases we will show $D_{0}=G_{0}$, by induction. Let $W_{1}=C_{V}\left(E_{0}\right) \perp V_{1} \perp \cdots \perp V_{k-1}$ and $W_{2}=C_{V}\left(E_{0}\right) \perp V_{2} \perp \cdots \perp V_{k}$. Inductively, $\mathscr{g}\left(W_{1}\right), \mathscr{f}\left(W_{2}\right) \leqslant D_{0}$ (here $\mathscr{f}\left(W_{i}\right)=\mathscr{G}\left(W_{i}\right)$ as defined in $\S 3$ ) and by (3.1) 
applied to the decomposition $V=V_{1} \perp\left(W_{1} \cap W_{2}\right) \perp V_{k}$ we conclude that either $G_{0} \cong S O^{+}(6,2)^{\prime}$ or $W_{1} \cap W_{2}$ contains no isotropic (singular) 1-space. The former is out as $\operatorname{dim}(V) \geqslant 8$ in the orthogonal cases. Therefore $W_{1} \cap W_{2}$ contains no isotropic (singular) 1-space. This is impossible for $V$ a symplectic space. If $V$ is unitary then $\operatorname{dim}\left(W_{1} \cap W_{2}\right)=1$, forcing $C_{V}\left(E_{0}\right)=0, k=3$, and $\operatorname{dim}\left(V_{i}\right)=1$ for $i=1,2,3$. If $V$ is orthogonal then $\operatorname{dim}\left(W_{1} \cap W_{2}\right) \leqslant 2$, forcing $\operatorname{dim}(V)<8$, a contradiction.

Suppose then that $G_{0} \cong S U(3, q)$. The proof of (4.2)(iv) shows that $g\left(V_{1} \perp V_{2}\right)$ and $\mathcal{G}\left(V_{2} \perp V_{3}\right)$ are each in $D_{0}$. So by the results of [5] and [14], $q=2$ and $p=3$, which is against our assumption.

To complete the proof of (4.3) we now assume $q=2, p=3$, and $E_{0}$ elementary abelian. As above we are done by (3.1) if $g\left(W_{1}\right)$ and $g\left(W_{2}\right)$ are each in $D_{0}$ (we are reduced to $G_{0} \cong S U(3,2)$, where an easy check gives the result). Suppose then that $g\left(W_{1}\right) \$ D_{0}$. As $E_{0}$ is elementary each $V_{i}$ is isomorphic to $Z_{2} \times Z_{2}$. If $G_{0}$ is symplectic or orthogonal, then each $V_{i}$ is a 2-space over $\mathbf{F}_{2}$, while if $V$ is unitary each $V_{i}$ is a 1-space over $\mathbf{F}_{4}$. So the decomposition is admissible. Let $I$ be the stabilizer in $G_{0} E_{0}$ of $\left\{C_{V}\left(E_{0}\right), V_{1}, \ldots, V_{k}\right\}$. Then $E_{0} \leqslant O_{3}(I)=E_{1}$, with $E_{1}$ elementary abelian.

We are assuming $D_{0}<G_{0}$. Let $F$ be a hyperplane of $E_{0}$. We will show that either $C_{G_{0}}(F) \leqslant I$ or that (4.1)(iii) holds. In the course of the proof it will become evident how to construct examples where (4.1)(ii) or (4.1)(iii) holds. For $i=1, \ldots, k$ let $F_{i}=C_{F}\left(V_{i}\right)$ and $\hat{V}_{i}=C_{V}\left(F_{i}\right)$. So $\hat{V}_{i} \geqslant C_{V}\left(E_{0}\right) \perp V_{i}$ and $C_{G_{0}}(F)$ acts on $\hat{V}_{i}$ for $i=1, \ldots, k$. In addition, $\hat{V}_{i}$ is the sum of $C_{V}\left(E_{0}\right)$ and certain of the subspaces in $\left\{V_{1}, \ldots, V_{k}\right\}$. Let $I_{i}$ be the normalizer in $I$ of $\hat{V}_{i}$. It will suffice to show that either (4.1)(iii) holds or, for each $i=1, \ldots, k,\left.C_{G_{0}}(F)\right|_{\hat{v}_{i}} \leqslant\left. I_{i}\right|_{\hat{v}_{i}}$.

Fix $i \in\{1, \ldots, k\}$. Reorder, if necessary, so that $i=1$ and $\hat{V}_{1}=C_{V}\left(E_{0}\right) \perp V_{1} \perp \cdots \perp V_{m}$, where $1 \leqslant m \leqslant k$. First suppose $m=1$. If $F>F_{1}$, then $C_{G_{0}}(F)$ normalizes $\left[\hat{V}_{1}, F\right]=V_{1}$ and $C_{\hat{V}_{1}}(F)=C_{V}\left(E_{0}\right)$ so we easily have $\left.C_{G_{0}}(F)\right|_{\hat{v}_{1}} \leqslant\left. I_{1}\right|_{\hat{v}_{1}}$. This also holds if $F=F_{1}$ except if $G_{0}=O(V)^{\prime}, \operatorname{dim}\left(C_{V}\left(E_{0}\right)\right)=2$ and $C_{V}\left(E_{0}\right)$ contains nonzero singular vectors. But in this case $C_{G_{0}}(F)=C_{G_{0}}\left(F_{1}\right) \geqslant 9\left(\hat{V}_{1}\right)=S O^{-}(4,2)^{\prime} \cong A_{5}$. From here we obtain $D_{0}=G_{0}$ using induction and (3.1) applied to the decomposition

$$
V=V_{2} \perp\left(C_{V}\left(E_{0}\right) \perp V_{1}\right) \perp\left(V_{3} \perp \cdots \perp V_{k}\right) .
$$

So from now on assume $m \geqslant 2$.

Suppose $F=F_{1}$ so that $g\left(\hat{V}_{1}\right) \leqslant C_{G_{0}}(F) \leqslant D_{0}$. If $k>m+1$ set $W_{1}=\hat{V}_{1} \perp V_{m+1} \perp \cdots \perp V_{k-1}$ and $W_{2}=\hat{V}_{1} \perp V_{m+2} \perp \cdots \perp V_{k}$. Inductively, $\left.C_{G_{0}}(F)\right|_{\hat{v}_{1}} \neq\left. I_{1}\right|_{\hat{v}_{1}}$ implies $g\left(W_{1}\right), g\left(W_{2}\right) \leqslant D_{0}$, so, by $(3.1), D_{0}=G_{0}$. So now suppose $k=m+1$. As $F_{1}$ acts on $V_{k}=V_{m+1}$ as a cyclic group we necessarily have $E_{0} \cong Z_{3} \times Z_{3}$. At this stage a check of the action of $E_{0}$ on $V$ shows that for some proper subgroup, $X$, of $E_{0}, C_{G_{0}}(X)$ contains an element $g \in I$ with $V_{k}^{g} \neq V_{k}$. Then $D_{0} \geqslant\left\langle\mathscr{g}\left(\hat{V}_{1}\right), g\left(\hat{V}_{1}\right)^{g}\right\rangle=G_{0}$ by (3.1). This is a contradiction, so we now have $F>F_{1}$.

This implies that $C_{G_{0}}(F)$ acts on $\left[\hat{V}_{1}, F\right]=V_{1} \perp \cdots \perp V_{m}$ and on $C_{\hat{V}_{1}}(F)=C_{V}\left(E_{0}\right)$. If $m=2$, then an easy check of the group of isometries of $V_{1} \perp V_{2}\left(S p(4,2), G U(2,2)\right.$, or $\left.O^{+}(4,2)\right)$ shows that $\left.C_{G_{0}}(F)\right|_{\hat{v}_{1}} \leqslant\left. I_{1}\right|_{\hat{v}_{1}}$. So we now 
suppose $m \geqslant 3$. We note that if $E_{0}=E_{1}$, then order considerations show that $m \leqslant 2$, so here (4.1)(ii) holds.

Suppose $G_{0}$ is unitary. Then assuming $\left.\left.C_{G_{0}}(F)\right|_{\hat{v}_{1}} \$ I_{1}\right|_{\hat{v}_{1}}, F$ must have an eigenspace on $V_{1} \perp \cdots \perp V_{m}$ of dimension at least 3, say $V_{1} \perp \cdots \perp V_{l}$. Then consider decompositions of $V$ of the form $V=U_{1} \perp\left(V_{1} \perp V_{2} \perp V_{3}\right) \perp U_{2}$, where $U_{1}$ and $U_{2}$ are each sums of some of the subspaces $\left\{C_{V}\left(E_{0}\right), V_{4}, \ldots, V_{k}\right\}$. By minimality of $G_{0}$ and the fact that $f\left(V_{1} \perp V_{2} \perp V_{3}\right) \leqslant C(F)$ we conclude that, for $i=1,2, D_{0}$ contains $\mathscr{g}\left(U_{i} \perp V_{1} \perp V_{2} \perp V_{3}\right)$, unless $V=U_{i} \perp V_{1} \perp V_{2} \perp V_{3}$. So by (3.1) we are done except in the case $k=4$ and $C_{V}\left(E_{0}\right)=1$. Here we can write $E_{0}=F \times E_{2} \cong Z_{3} \times Z_{3}$, where $E_{2}$ is trivial on $V_{1} \perp V_{2} \perp V_{3}$ and fixed-point-free on $V_{4}$. It is then easy to produce a maximal subgroup $E_{3}$ of $E_{0}$ inducing scalars on $V_{3} \perp V_{4}$. Therefore $C_{G_{0}}\left(E_{3}\right)$ contains an element $g$ interchanging $V_{3}$ and $V_{4}$, while stabilizing $V_{1} \perp V_{2}$. So

$$
\begin{aligned}
& D_{0} \geqslant\left\langle\mathscr{G}\left(V_{1} \perp V_{2} \perp V_{3}\right), \mathscr{G}\left(V_{1} \perp V_{2} \perp V_{3}\right)^{g}\right\rangle \\
& =\left\langle 9\left(V_{1} \perp V_{2} \perp V_{3}\right), \mathscr{G}\left(V_{1} \perp V_{2} \perp V_{4}\right)\right\rangle
\end{aligned}
$$

and (3.1) yields a contradiction. So $G_{0}$ is not a unitary group.

In the other cases $g\left(V_{1} \perp V_{2} \perp V_{3}\right) \cong S p(6,2)$ or $S O^{-}(6,2)^{\prime}$. Now $S p(6,2) \geqslant S O^{-}(6,2)^{\prime} \cong P S U(4,2) \cong P S p(4,3)$ and from here we check that in either case $C(F) \cap \mathscr{G}\left(V_{1} \perp V_{2} \perp V_{3}\right)$ contains $G U(3,2)$. We then argue as in the preceding paragraph with a decomposition $V=U_{1} \perp\left(V_{1} \perp V_{2} \perp V_{3}\right) \perp U_{2}$. Set $W_{i}=U_{i} \perp V_{1} \perp V_{2} \perp V_{3}$ for $i=1,2$.

In the orthogonal case apply (3.1), induction, and use the fact that $C(F) \cap$ if $\left(V_{1} \perp V_{2} \perp V_{3}\right) \leqslant C_{i\left(W_{1}\right)}^{0}\left(E_{0}\right)$. We reduce to the case $k=3$ or 4 . In the latter case $\left(V_{1} \perp V_{2} \perp V_{3}\right) \leqslant D_{0}$ by induction and a direct calculation produces a hyperplane $X$ of $E_{0}$ and element $g \in C(X) \cap I$ with $\left\{V_{1}, V_{2}, V_{3}, V_{4}\right\}^{g}=$ $\left\{V_{1}, V_{2}, V_{3}, V_{4}\right\}$ and $V_{4}^{g} \neq V_{4}$. As before this leads to $D_{0}=G_{0}$. If $k=3$, then as $\operatorname{dim}(V) \geqslant 8$ we necessarily have $\operatorname{dim}\left(C_{V}\left(E_{0}\right)\right)=2$ and $E_{0} \cong Z_{3} \times Z_{3}$. Inductively, if $\left(V_{1} \perp V_{2} \perp V_{3}\right) \leqslant D_{0}$. Also there is an element $e \in E_{0}^{\#}$ with $C(e)$ permuting $\left\{C_{V}\left(E_{0}\right), V_{1}, V_{2}, V_{3}\right\}$ and moving $C_{V}\left(E_{0}\right)$. Then (3.1) implies that $D_{0}=G_{0}$.

Now suppose that $G \cong S p(2 n, 2)$. Suppose $W_{1}, W_{2}<V$. Then by induction $C_{\dot{q}\left(W_{i}\right)}^{0}\left(E_{0}\right)=S p\left(W_{i}\right)$ or $O^{+}\left(W_{i}\right)$, where the form is described as in (4.1)(iii). By (3.1) we then have $D_{0} \geqslant O^{\varepsilon}(2 n, 2)$, where $\varepsilon=(-1)^{n}$. But $O^{\varepsilon}(2 n, 2)$ is maximal in $G_{0}$ (indeed $G_{0}$ is 2-transitive on the cosets of $O^{\varepsilon}(2 n, 2)$ ), so $D_{0}=G_{0}$ or $D_{0}=O^{\varepsilon}(2 n, 2)$. Thus we again reduce to the situation of $k=3$ or 4 . Also, $C_{V}\left(E_{0}\right)=0$ in the symplectic case, so $G_{0} \cong S p(6,2)$ or $S p(8,2)$. In the latter case we argue as before, so we are left with $G_{0} \cong S p(6,2)$, a group of order $2^{9} \cdot 3^{4} \cdot 5 \cdot 7$.

Here $C_{G_{0}}(F) \cong G U(3,2)$ and $F_{1}=1$. Viewing $E_{0} \leqslant g\left(V_{1}\right) \times g\left(V_{2}\right) \times g\left(V_{3}\right)$ we easily have $\left\langle\mathscr{g}\left(V_{1}\right) \times \mathscr{g}\left(V_{2}\right) \times \mathscr{f}\left(V_{3}\right), G U(3,2)\right\rangle=Z \leqslant D_{0}$. Checking a Sylow 2-subgroup of $Z$ (which must contain $Q_{8}$ and an $E_{8}$ ) we have $|Z|$ divisible by $2^{5} 3^{4}$. A Sylow 3-subgroup of $Z$ has the following orbits on $V^{\#}$ :

$$
\begin{aligned}
& O_{1}=\left\{v_{i}: v_{i} \in V_{i}^{\#}, i=1,2,3\right\}, \\
& O_{2}=\left\{v_{i}+v_{j}: i \neq j, v_{i} \in V_{i}^{\#}, v_{j} \in V_{j}^{\#}\right\}
\end{aligned}
$$


and

$$
O_{3}=\left\{v_{1}+v_{2}+v_{3}: v_{1} \in V_{1}^{\#}, v_{2} \in V_{2}^{\#}, v_{3} \in V_{3}^{\#}\right\}
$$

These have sizes 9, 27, 27 respectively. Suppose $D_{0}$ is transitive on $V^{\#}$. Then $D_{0}$ is transitive on the transvections of $G_{0}$ and as $D_{0}$ contains transvections, $G_{0}=D_{0}$, a contradiction. So $D_{0}$ is not transitive on $V^{\#}$. Since elements of order 7 in $G_{0}$ are fixed-point-free on $V^{\#}$ this implies $7 \nmid\left|D_{0}\right|$. Suppose 5||$D_{0} \mid$. An element of order 5 in $G_{0}$ fixes just 3 elements in $V^{\#}$, the nonzero vectors of a nondegenerate 2space. Therefore either $O_{1} \cup O_{2}$ or $O_{1} \cup O_{3}$ is an orbit of $D_{0}$ on $V^{\#}$. Let $g \in D_{0}$, $|g|=5$ and $v_{1}^{g}=v_{1}$. If $O_{1} \cup O_{2}$ is an orbit of $D_{0}$, then $g$ acts on $\left(O_{1} \cup O_{2}\right) \cap v_{1}^{\perp}=$ $\left\{v_{1}, V_{2}^{\#}, V_{3}^{\#}, v_{1}+V_{2}^{\#}, v_{1}+V_{3}^{\#}, V_{2}^{\#}+V_{3}^{\#}\right\}$. But this set contains 22 elements so $g$ fixes an additional element of this set. This is a contradiction. Therefore if 5||$D_{0} \mid$ the orbits of $D_{0}$ are $O_{1} \cup O_{3}$ and $O_{2}$. But this just says that $D_{0}$ preserves the quadratic form taking the value 1 on $V_{1}^{\#} \cup V_{2}^{\#} \cup V_{3}^{\#}$. That is $D_{0} \leqslant O^{-}(6,2)$ and has order divisible by $2^{5} \cdot 3^{4} \cdot 5$. Consequently $D_{0}=O^{-}(6,2)$. So now suppose $5 \nmid\left|D_{0}\right|$. Then $D_{0}$ is a $\{2,3\}$-group. No 2-local subgroup of $S p(6,2)$ has order divisible by $3^{4}$, so $D_{0}$ is 3-local, but not 2-local. Easy arguments show that $F\left(D_{0}\right)$ must contain an elementary abelian subgroup of order $3^{3}$, and this is not consistent with $D_{0} \geqslant G U(3,2)$. Thus $D_{0} \leqslant I$.

We have now completed the proof of (4.3) except for the construction of examples where (4.1)(iii) holds. Just argue as follows. Write $V=V_{1} \perp \cdots \perp V_{k}$ with $k \geqslant 3$ and set $\left\langle x_{i}\right\rangle=O_{3}\left(9\left(V_{i}\right)\right)$. Let $E_{0}=\left\langle x_{1} x_{2}^{-1}, x_{1} x_{2} x_{3}, x_{i}: i \geqslant 4\right\rangle$. Then $\left|E_{0}\right|=3^{k-1}$ and maximal subgroups of $E_{0}$ have order $3^{k-2}$. Given any such maximal subgroup, say $F$, there cannot exist a 4-set $\{i, j, l, m\}$ of $\{1, \ldots, k\}$ with $F_{i}=F_{j}=F_{l}=F_{m}$. For this contradicts $|F|$. The above arguments then imply that $C_{G_{0}}(F) \leqslant O^{\varepsilon}(2 k, 2)$ with $\varepsilon=(-1)^{k}$. By choice of $X$, (4.1)(i) fails, so we must have $D_{0}=O^{\varepsilon}(2 k, 2)$. This completes the proof of (4.3).

(4.4) $k>2$.

Proof. Suppose $k=2$ and notice that this implies $E_{0}$ has rank 2 . The proof here will follow from (3.2) once certain small cases are handled. If $G_{0} \cong S U(3, q)$, then $\operatorname{dim}\left(C_{V}\left(E_{0}\right)\right)=\operatorname{dim}\left(V_{1}\right)=\operatorname{dim}\left(V_{2}\right)=1$ and the result follows from the results of [5] and [14] (recall, $G$ simple implies $G_{0} \neq S U(3,2)$ ). Also the case of $S p(4,2)$ is easily checked. Suppose $C_{V}\left(E_{0}\right) \neq 0$. Then minimality of $G_{0}$ implies $g\left(V_{1} \perp V_{2}\right) \leqslant D_{0}$, so by (3.1) and (4.2)(iv) we are done. So assume $C_{V}\left(E_{0}\right)=0$.

Let $\left\langle e_{i}\right\rangle=C_{E_{0}}\left(V_{i}\right)$ for $i=1,2$. Recall that from (4.2)(iv) we have $g\left(V_{i}\right) \leqslant D_{0}$ for $i=1,2$. We first note that $E$ can be embedded in a maximal torus of $\bar{G}$. To see this choose $e \in E_{0}$ with $E_{0}=\left\langle e_{1}\right\rangle\langle e\rangle$. Then $e_{1} \in C_{\hat{G}}(e)$, so $e_{1}$ can be embedded in a $\sigma$-invariant maximal torus of $C_{\hat{G}}(e)=C_{\hat{G}}(e)^{0}$, which is a maximal torus of $\hat{G}$. Now pass to $\bar{G}$ to get the assertion. From (2.11) we have the existence of an element $f \in E-\left(\left\langle\bar{e}_{1}\right\rangle \cup\left\langle\bar{e}_{2}\right\rangle\right)$ such that $O^{r^{\prime}}\left(C_{G}(f)\right) \neq 1$. If $f_{0} \in E_{0}$ with $\bar{f}_{0}=f$, then $Q=$ $O^{r^{\prime}}\left(C_{G_{0}}\left(f_{0}\right)\right) \neq 1$, although $O^{r^{\prime}}\left(C\left(f_{0}\right) \cap\left(f\left(V_{1}\right) \times f\left(V_{2}\right)\right)\right)=1$.

Assume $(p, q) \neq(3,2)$. Then $Q \neq S_{3}$, so $Q$ does not stabilize $\left\{V_{1}, V_{2}\right\}$ and we can apply (3.2) to conclude $D_{0}=G_{0}$, except for a minor adjustment needed in case $G_{0}$ is 
an orthogonal group. Namely, (3.2) is stated for $X=O^{ \pm}(V)$, rather than for $G_{0}=\mathscr{g}(V)$. This was done to ease the proof of that result. In our situation consider $O^{+}(V)^{\prime}=G_{0} \unlhd O^{+}(V)=X . \quad E_{0}=\Omega_{1}(P)$ for $P \in \operatorname{Syl}_{p}\left(G_{0}\right)$, so the Frattini argument gives $X=G_{0} N_{X}\left(E_{0}\right)$. Now $N_{X}\left(E_{0}\right) \leqslant N_{X}\left(D_{0}\right)$, so $D_{0} N_{X}\left(E_{0}\right)$ is a subgroup of $X$ properly containing $C_{X}\left(V_{1}\right)$ and $C_{X}\left(V_{2}\right)$. So (3.2) implies $X=D_{0} N_{X}\left(E_{0}\right) \leqslant N_{X}\left(D_{0}\right)$, and hence $D_{0}=G_{0}$.

Finally, assume $(p, q)=(3,2)$. Here $G_{0} \cong S U(6,2), \quad E_{0} \cong Z_{3} \times Z_{9}$, and $\hat{G} \cong S L(6, K)$. If $f \in E_{0}$ and $|f|=9$, then $f$ has three distinct eigenvalues on $K \otimes V$ and so $C_{\hat{G}}(f) \cong S L(2, K) \times S L(2, K) \times S L(2, K)$. So $Q=O^{r^{\prime}}\left(C_{G_{0}}(f)\right) \not \nexists S_{3}$ and the earlier argument shows that $D_{0}=G_{0}$. This completes the proof of (4.4).

At this point the proof of (4.1) is complete and to complete the proof of Theorem 1 for $G$ a classical group we must consider the case where $E_{0}$ is nonabelian. To this end we assume that Theorem 1 holds for groups of order less than $|G|$.

We assume $E_{0}$ nonabelian. Then $E_{0}^{\prime} \cong Z_{p}$ and we may write $E_{0}=E_{1} E_{2} E_{3}$, where $E_{1}$ is 1 or extraspecial of exponent $p, E_{2}=Z\left(E_{0}\right)$, and $E_{3}=1$ or $E_{3}$ is nonabelian with a maximal cyclic subgroup. Since $p$ is odd, $G_{0}=S L(n, q)$ or $S U(n, q)$. The main result is the following.

(4.5) One of the following holds:

(i) $D_{0}=G_{0}$.

(ii) $G_{0} \cong S L\left(3^{k}, 4\right)$ and $p=3$.

(iii) $G_{0} \cong S U(n, 2)$ and $p=3$.

In cases (ii) and (iii) there is a group $F_{0} \leqslant G_{0}$ such that $F_{0} \geqslant Z\left(G_{0}\right), F_{0} / Z\left(G_{0}\right)$ is an elementary abelian 3-group and $C_{G_{0}}^{0}\left(F_{0}\right) \leqslant N_{G_{0}}\left(F_{0}\right)$.

We will prove (4.5) in several steps. Assume the result false and let $G$ be a minimal counterexample.

(4.6) $E_{0}$ acts irreducibly on $V$.

Proof. Suppose false and let $V_{1}$ be a proper $E_{0}$-invariant subspace of $V$ with $V_{1}$ irreducible. By (2.3), $G_{0} \neq S L(n, q)$ and $\operatorname{rad}\left(V_{1}\right)=0$. Then $V=V_{1} \perp V_{1}^{\perp}$ is $E_{0}$-invariant. Continue in this way, obtaining $V=V_{1} \perp \cdots \perp V_{k}$, with each $V_{i}$ $E_{0}$-invariant and irreducible. As each $V_{i}$ is a faithful module for $E_{1} E_{3}$ we have $\operatorname{dim}\left(V_{i}\right)=k_{i} p^{a}$, where $\left|E_{0}: E_{2}\right|=p^{2 a}$. We may assume $q>2$, for otherwise $Z\left(G_{0}\right) \cong Z_{3}, \quad p=3$, and (iii) holds. Suppose $k>2$. By minimality $\mathcal{G}\left(V_{1} \perp \cdots \perp V_{k-1}\right)$ and $\mathcal{g}\left(V_{2} \perp \cdots \perp V_{k}\right)$ (notation as before) are each in $D_{0}$, so we have $D_{0}=G_{0}$ by (3.1). Therefore we assume $k=2$.

Let $\langle z\rangle=\Omega_{1}\left(Z\left(E_{0}\right)\right)$ and let $g$ be the element of $G U(V)$ that is trivial on $V_{1}$ and induces $z$ on $V_{2}$. Then $\left[E_{0}, g\right]=1,|g|=p$, and we may assume $g \in E_{0}$ (see (2.9)). Let $e$ be an element of order $p$ in $E_{1} E_{3}-Z\left(E_{0}\right)$ and set $F=\langle e\rangle \times\langle g\rangle$. Now consider $C_{G_{0}}^{r}(F)$. Notice that $q \neq 2, q \neq 3$ (as $p \mid q+1$ ), and $q=4$ implies $p=5$. Since $E_{0}$ centralizes $F$ modulo $Z\left(G_{0}\right)$ we can apply induction, (2.6), and (2.16) to conclude that $C_{G_{0}}^{r}(F) \leqslant D_{0}$. Suppose that $C_{G_{0}}^{r}(F)$ stabilizes $\left\{V_{1}, V_{2}\right\}$. As $q \geqslant 4,(2.6)$ implies that $C_{G_{0}}^{r}(F)$ has no subgroup of index 2 and so $C_{G_{0}}^{r}(F)$ acts on $V_{1}$ and on $V_{2}$. But checking the eigenspaces of $e$ on $V$ we see that $O^{r^{\prime}}\left(C_{G_{0}}(e)\right)$ does not fix $V_{1}$ and $V_{2}$. This is a contradiction. Therefore $C_{G_{0}}^{r}(F)$ does not stabilize $\left\{V_{1}, V_{2}\right\}$, neither does $D_{0}$, and by (3.2) we have $D_{0}=G_{0}$. This proves (4.6). 
In view of (4.6) we now have $E_{0}$ irreducible on $V$, and consequently $E_{2}$ is cyclic. From the representation theory of $E_{0}$ we have $\operatorname{dim}(V)=p^{a}$ or $p^{a+1}$, where $\mid E_{1} E_{3}$ : $Z\left(E_{1} E_{3}\right) \mid=p^{2 a}$ (use the fact that $E_{2}^{p} \leqslant Z\left(G_{0}\right)$ ). In fact, $\operatorname{dim}(V)=p^{a}$ if and only if $E_{2}$ is diagonalizable on $V$. In view of these facts we now assume $q>4$ if $G_{0} \cong S L(n, q)$ and $q \geqslant 4$ if $G_{0} \cong S U(n, q)$.

(4.7) (i) $\operatorname{dim}(V)=p$;

(ii) $\left[E_{2}, G_{0}\right]=1$; and

(iii) $E_{1} E_{3}=E_{1}$ or $E_{3}$, with $\left|E_{1} E_{3}: Z\left(E_{1} E_{3}\right)\right|=p^{2}$.

Proof. Suppose $\operatorname{dim}(V) \geqslant p^{2}$. Choose $e \in E_{1} E_{3}-Z\left(E_{1} E_{3}\right)$ with $|e|=p$. From the representation theory of $E_{0}$ we see that $\langle e\rangle$ induces a multiple of the regular representation on $V$. Write $V=V_{1} \oplus \cdots \oplus V_{p}$, where $\left\{V_{1}, \ldots, V_{p}\right\}$ is the collection of eigenspaces of $e$. Choose $f$ of order $p$ such that $\langle e, f\rangle$ is extraspecial of order $p^{3}$. Redefining $E_{1}$, if necessary, we may assume $f \in E_{1}$ and write $E_{1} E_{3}=\langle e, f\rangle E_{4}$ with $\left[\langle e, f\rangle, E_{4}\right]=1$.

If $G_{0}=S L(V)$, then $\langle e, f\rangle$ reducible on $V$ implies $\langle e, f\rangle$ normalizes a parabolic subgroup of $G_{0}$. So (2.3) implies that $C_{G_{0}}^{r}(\langle e, f\rangle)=G_{0}$. Then induction, (2.6), and (2.16) imply that $G_{0}=C_{G_{0}}^{r}(\langle e, f\rangle) \leqslant D_{0}$. So we now assume $G_{0} \cong S U(V)$. Now $\langle f\rangle$ transitively permutes $\left\{V_{1}, \ldots, V_{p}\right\}$ and an easy computation shows that $V=V_{1} \perp \cdots \perp V_{p}$

Choose $g \in E_{4}-Z\left(E_{0}\right)$. Then $\langle e, g\rangle$ is abelian of rank 2 . The inductive argument of the last paragraph will work once we show $C_{G_{0}}^{r}(\langle e, g\rangle)=G_{0}$. For this first consider $\langle e, g\rangle$ acting on $V_{1} \perp V_{2}$. We have $g\left(V_{1}\right) \times g\left(V_{2}\right) \leqslant C_{G_{0}}^{r}(\langle e, g\rangle)$. The element $g$ has $p$ eigenvalues on $V$ and $\operatorname{dim}\left(V_{1} \perp V_{2}\right) \geqslant 2 p$. It follows that $O^{r^{\prime}}(C(g)) \cap \mathscr{g}\left(V_{1} \perp V_{2}\right)$ does not stabilize $\left\{V_{1}, V_{2}\right\}$. Consequently $g\left(V_{1} \perp V_{2}\right) \leqslant C_{G_{0}}^{r}(\langle e, g\rangle)$ by (3.2). Similarly, $q\left(V_{i} \perp V_{j}\right) \leqslant C_{G_{0}}^{r}(\langle e, g\rangle)$ for each $i \neq j$. Now, repeated use of (3.1) gives $G_{0}=C_{G_{0}}^{r}(\langle e, g\rangle)$. We have now proved that $\operatorname{dim}(V)=p$, so (i) holds. Since $E_{1} E_{3}$ is absolutely irreducible on $V$, (ii) follows. Also, (iii) follows from (i).

In view of (4.7)(iii) we alter our notation, if necessary, so that $E_{0}=E_{1} E_{2}$, where $\left[E_{2}, G_{0}\right]=1,\left|E_{1}: Z\left(E_{1}\right)\right|=p^{2}$, and $E_{1}$ acts irreducibly on $V$.

(4.8) $E_{1} E_{3}=E_{3}$.

Proof. Assume $G_{0} \cong S L(p, q)$ or $S U(p, q)$ and $E_{1} E_{3} \neq E_{3}$. Then $E_{1} E_{3}=E_{1}=\langle e, f\rangle$, where $|e|=|f|=p$. Recall that $q \geqslant 4$ and $q>4$ if $G_{0} \cong S L(n, q)$. As $p \mid q-1$ or $p \mid q+1$ according to $G_{0} \cong S L(V)$ or $S U(V)$ we can diagonalize $e$ and $f$. Write $V=W_{1} \oplus \cdots \oplus W_{p}$, where $W_{i}=\left\langle v_{i}\right\rangle$ is the eigenspace for eigenvalue $\alpha^{i-1}$ of $e$. Here $\alpha$ is an element of order $p$ in $\mathbf{F}_{q}^{\#}\left(\mathbf{F}_{q^{2}}^{\#}\right.$ in the unitary case), and $f$ may be chosen so that $v_{i}^{f}=v_{i+1}$ for $i=1, \ldots, p-1$. We get a basis of eigenvectors for $f$ by setting $w_{1}=v_{1}+\cdots+v_{p}$ and $w_{i}=w_{i-1}^{e^{-1}}$ for $i=2, \ldots, p$. Then $w_{i}^{f}=\alpha^{i-1} w_{i}$.

Now $C_{G_{0}}(e)$ and $C_{G_{0}}(f)$ stabilize the eigenspaces of $e$ and $f$, respectively. So $C_{G_{0}}(e), C_{G_{0}}(f)$ consists of all diagonal matrices in $G_{0}$ with respect to the ordered bases $\left\{v_{1}, \ldots, v_{p}\right\},\left\{w_{1}, \ldots, w_{p}\right\}$. Choose $h \in C_{G_{0}}(e)$ having eigenvalues $\beta, \gamma, \delta, \ldots, \delta$ in the basis $\left\{v_{1}, \ldots, v_{p}\right\}$ and $k \in C_{G_{0}}(f)$ with eigenvalues $\varepsilon, \eta, \ldots, \eta$ in the basis $\left\{w_{1}, \ldots, w_{p}\right\}$. These elements must be chosen so that $\beta \gamma \delta^{p-2}=\varepsilon \eta^{p-1}=1$. 
Consider the basis $\left\{v_{1}, \ldots, v_{p-1}, w_{1}\right\}$ of $V$ and check that in this ordered basis $h, k$ are given by the following matrices:

$$
\begin{aligned}
& h=\left(\begin{array}{ccccccccc}
\beta & & & & & & & \\
& \gamma & & & & & & \\
& & \delta & & & & & \\
& & & \cdot & & & & \\
\beta-\delta & \gamma-\delta & 0 & . & . & . & 0 & \delta
\end{array}\right) \text {, }
\end{aligned}
$$

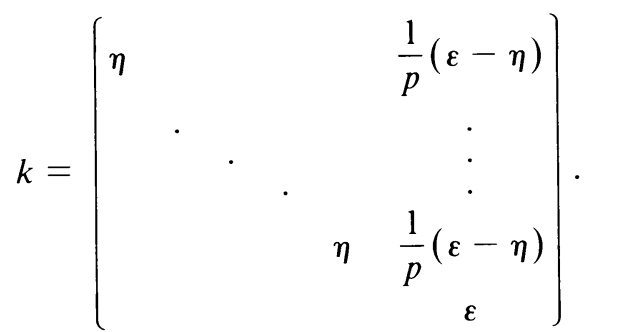

Notice that $h$ and $k$ each stabilize $V_{0}=\left\langle v_{1}, v_{2}, w_{1}\right\rangle$ and $V_{1}=\left\langle v_{3}-v_{4}, \ldots, v_{3}-v_{p}\right\rangle$, and $V=V_{0} \oplus V_{1}$ unless $p \equiv 2(\bmod r)$ and $V_{0} \cap V_{1}=\left\langle v_{3}+\cdots+v_{p}\right\rangle$. In the unitary case $\left[W_{i}, W_{j}\right]=0$ if $i \neq j$ and $\left[V_{0}, V_{1}\right]=0$.

Let $X$ be the subgroup of $G_{0}$ generated by all such elements $h, k$ above, and let $X_{0}$ and $X_{1}$ denote the restrictions of $X$ to $V_{0}$ and $V_{1}$, respectively. Then $X_{0}$ contains all matrices of the form

$$
\left(\begin{array}{ccc}
\beta & 0 & 0 \\
0 & \gamma & 0 \\
\beta-\delta & \gamma-\delta & \delta
\end{array}\right) \text { and }\left(\begin{array}{ccc}
\eta & 0 & \frac{1}{p}(\varepsilon-\eta) \\
0 & \eta & \frac{1}{p}(\varepsilon-\eta) \\
0 & 0 & \varepsilon
\end{array}\right)
$$

where $\beta \gamma \delta^{p-2}=\varepsilon \eta^{p-1}=1$. Considering the action of the elements $h$ above we see that an $X_{0}$-invariant subspace is necessarily a sum of the spaces $\left\langle v_{1}\right\rangle,\left\langle v_{2}\right\rangle$, $\left\langle v_{3}+\cdots+v_{p}\right\rangle$. Now consider the action of all possible elements $k$, with $k$ as above. We conclude that either $X_{0}$ is irreducible on $V_{0}$ or $\left\langle v_{3}+\cdots+v_{p}\right\rangle$ is invariant. In the latter case either $p \equiv 2(\bmod r)$ or $\varepsilon=\eta$ for all elements $k$ above, this forcing $p=q-1$ or $p=q+1$, depending on whether $G_{0} \cong S L(V)$ or $S U(V)$.

Suppose $X_{0}$ is irreducible on $V_{0}$, and consider the image $\bar{X}_{0}$ of $X_{0}$ in $\operatorname{PGL}(3, q)$ (respectively $P G U(3, q)$ ). Using the results of Bloom [5] and Hartley [14] we show that $\bar{X}_{0} \geqslant P S L(3, q)$ (respectively $\operatorname{PSU}(3, q)$ ). This involves checking the group $\bar{X}_{0}$ against the lists of groups presented in [5] and [14]. The following facts are useful in such a check. Let $I$ be the group of all elements $h$ above, restricted to $V_{0}$. Then $I \cong Z_{q \mp 1} \times Z_{q \mp 1}$ and has image in $\operatorname{PGL}(3, q)(\operatorname{PGU}(3, q))$ isomorphic to $\left(Z_{q \mp 1} \times Z_{q \mp 1}\right) / Z_{p}$. If $p=3, \bar{I}$ consists of matrices of determinant 1 , while if $p>3$, $\bar{I} \cap \operatorname{PSL}(3, q)(\bar{I} \cap \operatorname{PSU}(3, q))$ has index at most 3. Choosing $\bar{h} \in \bar{I}$ with $|h|=p$ and $h$ not scalar on $V_{0}$ we have $\bar{I} \leqslant C_{\bar{X}_{0}}(\bar{h})$. Comparing this with centralizers in the various proper subgroups of $\operatorname{PSL}(3, q)(\operatorname{PSU}(3, q))$, most cases are eliminated. Also 
one should recall that $\bar{X}_{0}$ is irreducible on $V_{0}$, that $\bar{k} \in \bar{I}$ only when $\bar{k}$ is scalar on $V_{0}$, and that when $p=3$ we may take $\bar{h}=\bar{e}$ and conclude that $C_{\bar{X}_{0}}(\bar{e})$ contains a subgroup isomorphic to $\bar{I}$, for any $e \in E_{1}-Z\left(E_{1}\right)$.

Once we have $\bar{X}_{0} \geqslant P S L(3, q)(P S U(3, q))$ we conclude that $X^{\prime}$ contains $\left(C\left(V_{1}\right) \cap N\left(V_{0}\right)\right)^{\prime}$. This is because $X$ induces a group of scalar matrices on $V_{1}$. Now the same argument can be carried out with $\left\langle v_{i}, v_{j}, w_{1}\right\rangle$ replacing $V_{0}$, where $i \neq j$ and $i, j \leqslant p-1$. It is now fairly easy to see that $D_{0}=G_{0}$. If $G_{0}=S U(p, q)$ this follows from repeated use of (3.1). For $S L(p, q)$ one can use $(B, N)$-pairs.

Next suppose $p \equiv 2(\bmod r)$. Here $r$ is odd and $V_{0} \cap V_{1}=\left\langle v_{3}+\cdots+v_{p}\right\rangle$. On $V_{0} /\left\langle v_{3}+\cdots+v_{p}\right\rangle X_{0}$ contains all matrices of the form

$$
\left(\begin{array}{ll}
\beta & 0 \\
0 & \gamma
\end{array}\right) \quad \text { and } \quad \frac{1}{2}\left(\begin{array}{ll}
\eta+\varepsilon & \eta-\varepsilon \\
\eta-\varepsilon & \eta+\varepsilon
\end{array}\right)
$$

where $\gamma=\beta^{-1}$ and $\eta^{p-1} \varepsilon=1$. Considering subgroups of $\operatorname{PSL}(2, q)$ we conclude that either $q \pm 1=2 p$ or $X_{0}$ induces on $V_{0} /\left\langle v_{3}+\cdots+v_{p}\right\rangle$ a subgroup of $G L(2, q)$ (respectively $G U(2, q)$ ), containing $S L(2, q)$. Excluding the case $q \pm 1=2 p$ and using the fact that $q$ is odd, we conclude $X_{0}^{(\infty)}$ contains a subgroup $Y \cong S L(2, q)$ and that there exists $V_{2}<V_{0}$ such that $V_{2}$ is $Y$-invariant and $V_{0}=V_{2} \oplus\left(V_{0} \cap V_{1}\right)$ (an orthogonal decomposition in the unitary case). As $X^{(\infty)}$ centralizes $V / V_{0}, C_{V}(Z(Y))$ is a complement to $V_{2}$ in $V$, and so $Y$ is generated by groups of transvections in $G_{0}$ (centers of long root subgroups). So by McLaughlin [19] or Wagner [28] we have $D_{0}=G_{0}$.

We are left with the case $p \equiv 2(\bmod r)$ and $q+1=2 p$, and the case $p=q \pm 1$. In the first case $G_{0}$ is unitary, $r=3$, and $q=3^{2^{b}}$ for some integer $b$, while in the second case we have $r=2$. In either case, let $\hat{V}_{0}=\left\langle v_{1}, v_{2}, w_{1}, w_{2}\right\rangle$ and let $Y$ be the subgroup of $D_{0}$ generated by all elements $h_{1}, h, k_{1}, k$, where $h_{1}, h$ are diagonal $(1,-1, \ldots,-1)$ and $\left(\gamma^{-1}, \gamma, 1, \ldots, 1\right)$ in the ordered basis $\left\{v_{1}, \ldots, v_{p}\right\}$, and $k_{1}, k$ are the same, but in the ordered basis $\left\{w_{1}, \ldots, w_{p}\right\}$.

For $\gamma \neq 1$, each of $h, h$ have fixed space in $\hat{V}_{0}$ of dimension 2. Indeed, $h$ centralizes $\left\langle v_{3}+\cdots+v_{p}, \alpha^{-2} v_{3}+\cdots+\alpha^{-(p-1)} v_{p}\right\rangle$ and $k$ centralizes $\left\langle w_{3}\right.$ $\left.+\cdots+w_{p}, \alpha^{-2} w_{3}+\cdots+\alpha^{-(p-1)} w_{p}\right\rangle$. Using these facts one checks that $Y$ is irreducible on $\hat{V}_{0}$. In fact, if $D$ is the subgroup of $Y$ generated by those $h, k$ for which $|\gamma|=p$, then $D$ is absolutely irreducible on $\hat{V}_{0}$ (consider eigenspaces of $h, k$ ). Now, $Y$ centralizes a $(p-4)$-subspace of $V$, so $V=\hat{V}_{0} \oplus \hat{V}_{1}$, with $Y$ trivial on $\hat{V}_{1}$. The sum is orthogonal in case $G_{0}$ is a unitary group. We identify $Y$ with its restriction to $\hat{V}_{0}$ and note that by McLaughlin [19] and Wagner [28] we may assume that $Y$ does not contain a full group of transvections.

First suppose that $p=q \pm 1$. Here we apply the result of Mwene [20] to get a contradiction, although a couple of remarks are in order. Firstly, for the $S U(4, q)$ case, we cannot simply apply Mwene's main theorem on subgroups of $S L\left(4, q^{2}\right)$, because that result only gives the maximal subgroups of $S L\left(4, q^{2}\right)$. However, the proof in [20] actually describes all subgroups of $S L\left(4, q^{2}\right)$. Secondly, in checking $Y$ against the proper subgroups of $S L(4, q)$ (resp. $\left.S L\left(r, q^{2}\right)\right)$, our previous remarks are sufficient to rule out all but one possibility-the case where $Y$ preserves a 
nondegenerate symplectic form on $\hat{V}_{0}$. So, suppose $Y$ fixes such a form, [, ]. Then $p v_{1}=w_{1}+\cdots+w_{p}$ implies that $0=\left[w_{3}+\cdots+w_{p}, p v_{1}-w_{1}-w_{2}\right]=\left[w_{3}\right.$ $\left.+\cdots+w_{p}, v_{1}\right]+\left[w_{3}+\cdots+w_{p}, w_{1}\right]+\left[w_{3}+\cdots+w_{p}, w_{2}\right]$. Checking eigenspaces of the elements $k$ above, we see that $w_{3}+\cdots+w_{p}$ must be orthogonal (under $\left.[],\right)$ to $\left\langle w_{1}, w_{2}\right\rangle$, and so $\left[w_{3}+\cdots+w_{p}, v_{1}\right]=0$. This implies $w_{3}+\cdots+w_{p} \in\left\langle v_{1}, v_{3}\right.$ $\left.+\cdots+v_{p}, \alpha^{-2} v_{3}+\cdots+\alpha^{-(p-1)} v_{p}\right\rangle$, which is false. So $Y$ fixes no such form, and the case $p=q \pm 1$ is out.

Suppose $q+1=2 p$. Choose $h$ as above with $|h|=q+1$ and write $h=h_{2} h_{p}$, where $\left|h_{2}\right|=2,\left|h_{p}\right|=p$, and $\left[h_{2}, h_{p}\right]=1$. Similarly, for $k=k_{2} k_{p}$. Then $D=\left\langle h_{p}, k_{p}\right\rangle$ and previous remarks show that $C_{I}(D) \leqslant Z(I) \cong Z_{2}$, where $I=S U(4, q)$. Since the Sylow $p$-subgroups of $I$ are abelian, this immediately implies $O_{p}(D)=1$.

Let $s \neq p$ be prime. We claim $O_{s}(Y) \leqslant Z(I) \cong Z_{2}$. Suppose otherwise, and let $J$ be minimal normal in $Y$ with $J \leqslant O_{s}(Y)$. As $D$ is absolutely irreducible, $s \neq r$ and one of $\left[J, h_{p}\right]$ or $\left[J, k_{p}\right]$ is nontrivial. By symmetry, we assume the former. If $s \neq 2$, then $\left[J, h_{p}\right]\left\langle h_{p}\right\rangle$ is a Frobenius group and standard arguments from representation theory imply $\operatorname{dim}\left(\hat{V}_{0}\right) \geqslant p$, a contradiction. The same argument can be used when $s=2$, unless $J$ is extraspecial. In the latter case, set $J_{0}=\left[J, h_{p}\right] \cap C\left(h_{2}\right)$. Then $J_{0} \$ Z(I)$ and $\left\langle h_{p}\right\rangle$ is fixed-point-free on $J_{0} / Z(I)$. But $h_{p}$ normalizes no such 2-group in $C_{I}\left(h_{2}\right)$. This proves the claim, from which it follows that $F^{*}(Y)=E(Y)$ or $E(Y) Z(I)$.

The group $C_{I}\left(h_{2}\right)$ satisfies $C_{I}\left(h_{2}\right)^{(\infty)} \cong S L(2, q) \circ S L(2, q)$, with $h$ in one of the factors and $h_{1}$ inducing an outer diagonal automorphism on this factor. From earlier assumptions, neither factor is contained in $Y$. Therefore, $\left\langle h_{p}\right\rangle \leqslant O_{p}\left(C_{Y}\left(h_{2}\right)\right)$.

The restriction $q+1=2 p$ forces $\operatorname{PSU}(4, q)$ to have sectional 2-rank 4. In particular, $E(Y)$ is the product of at most 2 components and if there are two, then they are each of sectional 2-rank 2 . Each of $h_{p}$ and $k_{p}$ must stabilize each component of $Y$, inducing a nontrivial automorphism on at least one component. From the above paragraph we conclude the components of $Y$ are normalized by $\langle h, k\rangle$.

By Gorenstein-Harada [31], the components of $Y$ have known structures. Also, we have $\left\langle h_{p}\right\rangle \leqslant O_{p}\left(C_{Y}\left(h_{2}\right)\right)$ and similarly for $k_{2}$. It follows (use [3] and Table 1 of [29]) that each component of $D$ is of Lie type in odd characteristic. Since $\operatorname{dim}\left(\hat{V}_{0}\right)=4$, the Sylow $s$-subgroups of $Y$ are abelian for all primes $s \neq 3$. Assume that $J$ is a component of $Y$ and of Lie type in characteristic $s>3$. By the above, $J \cong S L\left(2, s^{c}\right)$ or $\operatorname{PSL}\left(2, s^{c}\right)$. As $\operatorname{PSL}\left(2, s^{c}\right)$ contains a Frobenius group of order $\frac{1}{2} s^{c}\left(s^{c}-1\right)$, and since $\operatorname{dim}\left(\hat{V}_{0}\right)=4$, we must have $s^{c} \leqslant 9$. However, $p|| J \mid$ and $s \neq 3$. This forces $s^{c}=5=p$, and contradicts the facts $\left\langle h_{p}\right\rangle \leqslant O_{p}\left(C_{Y}\left(h_{2}\right)\right)$ and $\left\langle k_{p}\right\rangle \leqslant O_{p}\left(C_{Y}\left(k_{2}\right)\right)$.

At this point we have established that the components of $Y$ are of Lie type in characteristic 3. Each of $h_{p}$ and $k_{p}$ induce inner automorphisms on $E(Y)$. For otherwise, a field automorphism is induced and we obtain a contradiction, as before, by considering certain Frobenius groups. Therefore $h_{p}, k_{p} \in E(Y)$. If $Y$ has two components, $X_{1}$ and $X_{2}$, then each has the form $\operatorname{SL}\left(2,3^{e}\right)$ or $\operatorname{PSL}\left(2,3^{e}\right)$ and each 
has order divisible by $p$. As $X_{1}$ centralizes a Sylow 3-subgroup, $R$, of $X_{2}, R \times X_{2}$ is in a proper parabolic subgroup of $I$. One checks that this forces $R$ to consist of transvections and $X_{2}=S L\left(2,3^{e}\right)=S L(2, q)$ is generated by full groups of transvections. This is a contradiction. Thus, $E(Y)$ is quasisimple.

Suppose $E(Y) / Z(E(Y)) \cong P S L\left(2,3^{e}\right)$. Since $h_{p} \in E(Y), p \mid 3^{e} \pm 1$ and this forces $q \mid 3^{e}$ (use primitive divisors). As $C\left(h_{p}\right) \cap Y Z(I) \geqslant Z(I) \times\left\langle h_{2}\right\rangle \times\left\langle h_{1}\right\rangle$, and since $Y Z(I) / Z(I) \leqslant \operatorname{Aut}\left(P S L\left(2,3^{e}\right)\right)$, some element of $\left\langle h_{1}, h_{2}\right\rangle$ induces an involutory field automorphism of $E(Y)$. As $\left\langle h_{p}\right\rangle \leqslant O_{p}\left(C_{Y}\left(h_{2}\right)\right)$, this element cannot be $h_{2}$. Hence both $h_{1}$ and $h_{1} h_{2}$ induce field automorphisms. The containment $E(Y) \leqslant$ $S U(4, q)$, forces $3^{e}=q^{2}$, so $E(Y) \cong S L\left(2, q^{2}\right)$ or $P S L\left(2, q^{2}\right)$. Now, $E\left(C_{I}\left(h_{1}\right)\right)=$ $S U(3, q)$, with natural action on $\left\langle v_{1}\right\rangle^{\perp} \cap V_{0}$. We may write $J=O^{p^{\prime}}\left(E(Y) \cap C\left(h_{1}\right)\right)$ and $J=\left\langle h_{p}, h_{p}^{j}\right\rangle$, for some $j \in J$. Then $J \cong S L(2, q)$ or $\operatorname{PSL}(2, q)$. But $h_{p}$ is trivial on a 2 -space of $\left\langle v_{1}\right\rangle^{\perp} \cap V_{0}$, so $J$ is trivial on a 1-space. It then follows that $J \cong S L(2, q)$ and is generated by full groups of transvections. This is a contradiction.

The proper parabolic subgroups of $I$ have at most one noncyclic composition factor and this is isomorphic to $\operatorname{PSL}(2, q)$ or to $\operatorname{PSL}\left(2, q^{2}\right)$. It follows that $E(Y) / Z(E(Y)) \cong P S L\left(3,3^{e}\right), P S U\left(3,3^{e}\right), P S L\left(4,3^{e}\right), P S U\left(4,3^{e}\right)$, or $G_{2}\left(3^{e}\right)$ with $e$ a power of 2. Since $h_{p} \in E(Y)$, an order argument shows that either $a \mid e$ or $E(Y)$ is a 4-dimensional symplectic or unitary group with $a \mid 2 e$. As $E(Y)<I$, we use additional order arguments to conclude $E(Y) / Z(E(Y)) \cong P S U(3, q), P S p(4, q)$, or $P S p(4, \sqrt{q})$. In the first two cases, let $j$ be an involution in $E(Y)-Z(I)$ with $j(Z(E(Y))) 2$ central. Then $C_{E(Y)}(j)$ contains an $S L(2, q)$ component with center $\langle j\rangle$. This component is necessarily one of $C_{I}(j)$ (as $j \sim h_{2}$ in $\left.I\right)$, hence generated by full groups of transvections. We are assuming this false, so these cases are out. For the last case one argues that in $\operatorname{Aut}(P \operatorname{SP}(4, \sqrt{q}))$ the centralizer of an element of order $p$ is cyclic of order $2 p$. As $\left\langle h_{1}, h_{2}\right\rangle \leqslant C\left(h_{p}\right)$, there is some involution $h \in\left\langle h_{1}, h_{2}\right\rangle \cap C(E(Y))$. But then $C_{I}(j)$ has a section isomorphic to $P \operatorname{Sp}(4, \sqrt{q})$, whereas $E\left(C_{I}(j)\right) \cong S U(3, q)$ or $S L(2, q) \times S L(2, q)$. This is a contradiction, completing the proof of (4.8).

(4.9) $E_{1} E_{3} \neq E_{3}$.

Proof. Suppose $E_{1} E_{3}=E_{3}$ and write $E_{3}=\langle e, f\rangle$ with $|e|=p$ and $|f|=p^{n}$. Recall that $G_{0} \neq S L(3,4)$. Let $1 \neq \alpha$ be an eigenvalue of $e$ on $V$ and let $V_{i}=\left\langle v_{i}\right\rangle$ be the eigenspace of $e$ with corresponding eigenvalue $\alpha^{i-1}$. First suppose that $p^{n}|q-1|$ $\left(p^{n} \mid q+1\right.$ in the unitary case). Then $f$ is diagonalizable with eigenvalues $\beta, \beta \alpha, \ldots, \beta \alpha^{p-1}$, where $\beta$ has order $p^{n}$ in the multiplicative group of the underlying field $\left(\mathbf{F}_{q}\right.$ or $\mathbf{F}_{q^{2}}$ ). So there is a scalar transformation $z$ on $V$ such that $|f z|=p$ and $f z$ has distinct eigenvalues. Replacing $E_{3}$ by $\langle e, f z\rangle$ we reduce to (4.8). So from now on we may assume $p^{n} \nmid q-1\left(p^{n} \nmid q+1\right.$ in the unitary case $)$.

This forces $\langle f\rangle$ to act irreducibly on $V$. In an appropriate extension field, $f$ has the eigenvalues above, so $f \notin G_{0}$. Now $G L(p, q)=G_{0} Z\langle f\rangle(G U(p, q)=$ $\left.G_{0} Z\langle f\rangle\right)$, where $Z=Z(G L(p, q))(Z(G U(p, q)))$. So $H=C(f) \cap G L(p, q)$ (respectively, $H=C(f) \cap G U(p, q)$ ) is contained in $I=N\left(D_{0}\right)$. Indeed, $\left(H \cap G_{0}\right) Z\langle f\rangle \leqslant D_{0} Z\langle f\rangle \leqslant N\left(D_{0}\right)$. We may assume $I \neq S L(p, q)(I \neq S U(p, q))$. 
Now $H$ is cyclic of order $q^{p}-1\left(q^{p}+1\right)$, so $I$ is transitive on 1 -spaces of $V$ if $G_{0} \cong S L(p, q)$.

Let $W=C(e) \cap G L(p, q)$ (respectively $C(e) \cap G U(p, q)$ ) and $W_{1}=O_{p}(W)$. We claim that $W \leqslant I$. To see this first note that $W_{1} \cong\left(Z_{p^{n-1}}\right)^{p}$ and has index $p$ in a Sylow $p$-subgroup of $G L(p, q)(G U(p, q))$. In fact $W_{1}\langle f\rangle \cong Z_{p^{n-1}}$ wr $Z_{p}$ is a Sylow $p$-subgroup of $G_{0}\langle f\rangle$. As $W \leqslant G_{0} Z W_{1}$, it suffices to show $W_{1} \leqslant I$. Let $W_{0}=W_{1} \cap$ $G_{0}$, so $W_{0} \leqslant D_{0}$. Suppose $W_{0} \in \operatorname{Syl}_{p}\left(D_{0}\right)$. Since $O_{p}\left(Z\left(G_{0}\right)\right)<W_{0}$ we can apply easy transfer and fusion arguments to get $D_{0}=D_{1} \times O_{p}\left(Z\left(G_{0}\right)\right)$ for some subgroup $D_{1}$. Since $f$ normalizes $W_{0}$ but no proper subgroup of $W_{0}$ not containing $O_{p}\left(Z\left(G_{0}\right)\right)$ we must have $W_{0} \cap O^{p}\left(D_{0}\right)=1$. That is $D_{0}=O_{p^{\prime}}\left(D_{0}\right) W_{0}$. Let $s$ be a prime such that $s \mid q^{p}-1$ (resp. $\left.s \mid q^{2 p}-1\right)$ but $s \nmid r^{a}-1$ for $r^{a}<q$ (resp. $r^{a}<q^{2}$ ). Let $S \in$ $\operatorname{Syl}_{2}(H)$. Then $S \leqslant G_{0}$, so $S \leqslant D_{0}$, and a Frattini argument shows that $S$ is normalized by a conjugate of $W_{0}$. But $N(S)$ has $p$-rank 2 , so $m_{p}\left(W_{0}\right) \leqslant 2$. Since $m_{p}\left(W_{0}\right)=p-1$, this is a contradiction unless $p=3$. But $p=3$ is eliminated by considering the lists in Hartley [14] and Bloom [5]. So we now assume $p>3$ and $W_{0} \notin \operatorname{Syl}_{p}\left(D_{0}\right)$. If $W_{0}<\hat{W}_{0} \in \operatorname{Syl}_{p}\left(D_{0}\right)$, then there exists $g \in \hat{W}_{0}$ with $e^{g}=e z$ for some $1 \neq z \in Z\left(G_{0}\right)$. We may choose $z$ such that $e^{g f}=e$. Now take $h \in W_{1}-W_{0}$ such that $|f h|=p$. Then $\langle e, f h\rangle$ is extraspecial of exponent $p, f h \in G_{0}$, and $g f h \in C_{G_{0}}(e) \leqslant D_{0}$. So $g f h \in I$, and since $g, f \in I, h \in I$. But $W_{1}=\left\langle W_{0}, h\right\rangle$ implies that $W_{1} \leqslant I$ as claimed.

Suppose $G_{0} \cong S L(p, q)$. Let $K$ be the subgroup of $W$ that is trivial on $\left\langle v_{2}, \ldots, v_{p}\right\rangle$. Then $[V, K]=\left\langle v_{1}\right\rangle$. Let $A=\left\langle v_{1}, v_{2}\right\rangle$. As $I$ is transitive on 1 -spaces, for each 1 -space $\langle v\rangle \leqslant A$, there is a subgroup $K^{g} \leqslant I$ such that $\left[K^{g}, V\right]=\langle v\rangle$. Let $X$ be the subgroup of $I$ generated by all such $K^{g}$. Then $\left.X\right|_{A}$ contains all diagonal matrices in the basis $\left\{v_{1}, v_{2}\right\}$. It follows that $\left.X\right|_{A}$ contains all matrices of form $\left(\begin{array}{ll}1 & 0 \\ x & 1\end{array}\right)$ for $x \in \mathbf{F}_{q}$, where the matrices are taken with respect to either the ordered basis $\left\{v_{1}, v_{2}\right\}$ or the ordered basis $\left\{v_{2}, v_{1}\right\}$. In either case one can then argue that $X$ contains a full group of transvections. Therefore $D_{0}=G_{0}$ by McLaughlin [19].

We are left with the case $G_{0}=S U(p, q)$. The argument is similar to the above. As before let $K$ be the subgroup of $W$ centralizing $\left\langle v_{2}, \ldots, v_{p}\right\rangle$. Suppose that, for some $g \in I, \operatorname{rad}\left(\left\langle v_{1}, v_{1}^{g}\right\rangle\right)=0$ and $v_{1}^{g} \notin v_{1}^{\perp}$. Set $A=\left\langle v_{1}, v_{1}^{g}\right\rangle$. Then $X=\left\langle K, K^{g}\right\rangle$ acts on $A$ and on $A^{\perp}$. As $X$ centralizes $V / A$ and $V=A \perp A^{\perp}$ we conclude that $X$ is trivial on $A^{\perp}$. Now $K$ is transitive on the isotropic 1-spaces of $A$ (since for each element $1 \neq k \in K, k$ stabilizes only the spaces $\left\langle v_{1}\right\rangle$ and $C_{A}(K)$, neither of which is isotropic), so it follows that $\left.X\right|_{A} \geqslant S L(2, q)$. So $I$ contains a group of transvections and $I \geqslant G_{0}$ by Wagner [28]. This is a contradiction. So now suppose that $v_{1}^{g} \in v_{1}^{\perp}=\left\langle v_{2}, \ldots, v_{p}\right\rangle$ whenever $\operatorname{rad}\left(\left\langle v_{1}, v_{1}^{g}\right\rangle\right)=0$ and $g \in I$.

Recall that $C(f)=H \leqslant I$, and it is irreducible. It is then possible to choose $g \in I$ such that $v_{1}^{g} \notin\left\langle v_{1}\right\rangle$ and $v_{1}^{g} \notin v_{1}^{\perp}$. Consequently $\operatorname{rad}\left(\left\langle v_{1}, v_{1}^{g}\right\rangle\right) \neq 0$. So $v_{1}^{g}=\beta v_{1}+a$ for some $0 \neq a \in\left\langle v_{2}, \ldots, v_{p}\right\rangle$. Choose $i \geqslant 2$ such that $\left(a, v_{i}\right) \neq 0$, and set $A=\left\langle v_{1}, v_{1}^{g}, v_{i}\right\rangle$. Then $\operatorname{rad}(A)=0$. Now $C(e)$ contains a subgroup $I_{0}$ isomorphic to $Z_{q+1} \times Z_{q+1} \times Z_{q+1}$ such that $I_{0}$ is faithful and diagonalizable on $A$ and stabilizes $A^{\perp}$. Using the results of Hartley [14] and Bloom [5] we conclude that $X=\left\langle H_{0}, K^{g}\right\rangle$ contains $S L(2, q)$ (and generated by groups of transvections). As before the results of Wagner [28] imply $G_{0} \leqslant I$. This is a contradiction, proving (4.9). 
The proof of (4.5) will be complete once we show that for $G_{0} \cong S L\left(3^{k}, 4\right)$ and $S U(n, 2)$ there is a 3-group $E_{0}<G_{0}$ such that $C_{G_{0}}^{0}\left(E_{0}\right) \leqslant N_{G_{0}}\left(E_{0}\right)$. For $G_{0} \cong S U(n, 2)$ this follows from (4.1). So suppose $G_{0} \cong S L\left(3^{k}, 4\right)$. Let $E_{0}$ be an extraspecial 3-group of order $3^{2 k+1}$ and of exponent 3 . Then we can consider $E_{0}<G_{0}$. If $V$ is the module affording this representation, then each $e \in E_{0}-Z\left(E_{0}\right)$ has 3 distinct eigenspaces on $V$ of dimension $3^{k-1}$. Using this and the fact that $C_{E_{0}}(e)$ is absolutely irreducible on each of these eigenspaces, we conclude that $C_{G_{0}}^{0}\left(E_{0}\right) \leqslant N_{G_{0}}\left(E_{0}\right)$. This completes the proof of (4.5).

5. Exceptional groups of Lie type. In this section $G$ will denote either an exceptional group of Lie type or one of the groups $G_{2}(2)^{\prime}$ or ${ }^{2} F_{4}(2)^{\prime}$. Notation will be as in $\S 2$. Namely, $\bar{G}$ is a simple algebraic group with $G=O^{r^{\prime}}\left(\bar{G}_{\sigma}\right)^{\prime}$. By $(2.15), \bar{G}_{\sigma}$ is $G$ together with all diagonal automorphisms of $G$. We take $E \leqslant \bar{G}_{\boldsymbol{\sigma}}$ and define $\hat{G}, E_{0}$, $G_{0}$, etc. as in $\S 2$. By $(2.15)\left|\bar{G}_{\sigma}: G\right|$ divides $|Z(\hat{G})|$. So except for the cases $p=3$, $G \cong E_{6}(q)$ or ${ }^{2} E_{6}(q)$, with $3 \mid q-1$ or $3 \mid q+1$, respectively, we necessarily have $E_{0} \leqslant G_{0}$. We will prove

(5.1) With the above notation one of the following holds:

(i) $C_{G_{0}}^{0}\left(E_{0}\right)=G_{0}$.

(ii) $p=3$ and $G \cong G_{2}(2)^{\prime}, F_{4}(2), F_{4}(4), E_{6}(2), E_{6}(4),{ }^{2} E_{6}(2), E_{7}(2), E_{8}(2)$, or $E_{8}(4)$. If $G \cong G_{2}(2)^{\prime}$, then $D_{0}$ normalizes a Sylow 3-subgroup of $G$. In the other cases an example $E_{0}$ exists such that $C_{G_{0}}^{0}\left(E_{0}\right) \leqslant N_{G_{0}}\left(E_{0}\right)$.

(iii) $p=5, G={ }^{2} F_{4}(2)^{\prime}$, and $C_{G_{0}}^{0}\left(E_{0}\right) \leqslant N_{G_{0}}\left(E_{0}\right)$.

(iv) $p=3, G={ }^{2} F_{4}(2)^{\prime}$ and for $E_{0} \cong Z_{3} \times Z_{3}$ we have $C_{G_{0}}^{0}\left(E_{0}\right) \cong \operatorname{Aut}\left(L_{3}(3)\right)$. On the other hand if $G_{1}={ }^{2} F_{4}(2)$, then $C_{G_{1}}^{0}\left(E_{0}\right)=G_{1}$.

In this section we will prove (5.1). So assume (5.1) false and let $G$ be a counterexample of minimal order. Let $\Phi_{d}(x)$ denote the cyclotomic polynomial with roots the primitive complex $d$ th roots of 1 . Then set $\varphi_{d}=\Phi_{d}(q)$. The group $G$ and the order of $G_{0}$ is given in the following table.

TABLE (5.2)

\begin{tabular}{cl}
$G$ & $\left|G_{0}\right|$ \\
\hline$G_{2}(q)$ & $q^{6} \varphi_{6} \varphi_{3} \varphi_{2}^{2} \varphi_{1}^{2}$ \\
${ }^{3} D_{4}(q)$ & $q^{12} \varphi_{12} \varphi_{6}^{2} \varphi_{3}^{2} \varphi_{2}^{2} \varphi_{1}^{2}$ \\
${ }^{2} F_{4}(q)$ & $q^{12} \varphi_{12} \varphi_{6} \varphi_{4}^{2} \varphi_{2}^{2} \varphi_{1}^{2}$ \\
$F_{4}(q)$ & $q^{24} \varphi_{12} \varphi_{8} \varphi_{6}^{2} \varphi_{4}^{2} \varphi_{3}^{2} \varphi_{2}^{4} \varphi_{1}^{4}$ \\
${ }^{2} E_{6}(q)$ & $q^{36} \varphi_{18} \varphi_{12} \varphi_{10} \varphi_{8} \varphi_{6}^{3} \varphi_{4}^{2} \varphi_{3}^{2} \varphi_{2}^{6} \varphi_{1}^{4}$ \\
$E_{6}(q)$ & $q^{36} \varphi_{12} \varphi_{9} \varphi_{8} \varphi_{6}^{2} \varphi_{5} \varphi_{4}^{2} \varphi_{3}^{3} \varphi_{2}^{4} \varphi_{1}^{6}$ \\
$E_{7}(q)$ & $q^{63} \varphi_{18} \varphi_{14} \varphi_{12} \varphi_{10} \varphi_{9} \varphi_{8} \varphi_{7} \varphi_{6}^{3} \varphi_{5} \varphi_{4}^{2} \varphi_{3}^{3} \varphi_{2}^{7} \varphi_{1}^{7}$ \\
$E_{8}(q)$ & $q^{120} \varphi_{30} \varphi_{24} \varphi_{20} \varphi_{18} \varphi_{15} \varphi_{14} \varphi_{12}^{2} \varphi_{10}^{2} \varphi_{9} \varphi_{8}^{2} \varphi_{7} \varphi_{6}^{4} \varphi_{5}^{2} \varphi_{4}^{4} \varphi_{3}^{4} \varphi_{2}^{8} \varphi_{1}^{8}$
\end{tabular}

We will say $p$ is associated with $\varphi_{x}$ if $x$ is minimal subject to $p \mid \varphi_{x}$. Notice that if $x$ is even, then $\varphi_{x}$ divides $q^{x / 2}+1$. Also if $p$ is associated with $\varphi_{x}$, then $p>x$. The next several lemmas deal with the easiest cases. These are the cases where the $p$-rank of $G_{0}$ is 1 , where (2.3) applies, or where induction is easily applied. In effect, we reduce to the case where $p$ is a small prime divisor of $q \pm 1$. 
(5.3) Suppose $p$ is associated with $\varphi_{x}$. None of the following can occur: $G=G_{2}(q)^{\prime}$ and $x=3,6 ; G={ }^{3} D_{4}(q)$ and $x=12 ; G={ }^{2} F_{4}(q)^{\prime}$ and $x=6,12 ; G=F_{4}(q)$ and $x=8,12 ; G={ }^{2} E_{6}(q)$ and $x=12,18 ; G=E_{6}(q)$ and $x=9,12 ; G=E_{7}(q)$ and $x=14,18 ; G=E_{8}(q)$ and $x=15,20,24$, or 30 .

Proof. This follows from (2.7)(ii) and (2.7)(iv). It is necessary to check that $p \nmid|\hat{W}|$, and then check that $p$ does not divide the order of any maximal parabolic of $G$.

(5.4) Suppose $p$ is associated with $\varphi_{x}$. None of the following can occur: $G={ }^{2} E_{6}(q)$ and $x=8,10 ; G=E_{6}(q)$ and $x=4,5,8 ; G=E_{7}(q)$ and $x=3,4,5,7,8,9,10,12$; $G=E_{8}(q)$ and $x=7,9,14,18$.

Proof. The idea is as follows. Say, for example, that $G={ }^{2} E_{6}(q)$ and $x=8$ or 10 . From (5.2) we conclude that a Sylow $p$-subgroup of $G_{0}$ has order dividing $q^{4}+1$ or $q^{5}+1$, respectively. Also $G_{0}$ contains $S O^{-}(8, q)^{\prime}$ and $S U(6, q)$ and order considerations show that a Sylow $p$-subgroup of $G_{0}$ is contained in one of these subgroups. As each of the subgroups is contained in a proper parabolic subgroup of $G_{0}$, we are done by (2.3).

The same argument works for the other cases. Let $E_{0} \leqslant P \in \operatorname{Syl}_{p}\left(G_{0}\right)$. Below are the triples $\left(G, I, \varphi_{x}\right)$ where $P \in \operatorname{Syl}_{p}(I)$ and $I$ is involved in a proper parabolic subgroup of $G_{0}$.

\section{TABLE}

\begin{tabular}{lll}
$G$ & $I$ & $\varphi_{x}$ \\
\hline$E_{6}(q)$ & $L_{6}(q)$ & $\varphi_{5}$ \\
$E_{6}(q)$ & $S O^{+}(10, q)^{\prime}$ & $\varphi_{4}, \varphi_{8}$ \\
$E_{7}(q)$ & $E_{6}(q)$ & $\varphi_{3}, \varphi_{4}, \varphi_{9}, \varphi_{12}$ \\
$E_{7}(q)$ & $S O^{+}(12, q)^{\prime}$ & $\varphi_{5}, \varphi_{8}, \varphi_{10}$ \\
$E_{7}(q)$ & $L_{7}(q)$ & $\varphi_{7}$ \\
$E_{8}(q)$ & $E_{7}(q)$ & $\varphi_{7}, \varphi_{9}, \varphi_{14}, \varphi_{18}$
\end{tabular}

(5.5) Suppose $p$ is associated with $\varphi_{1}$ or $\varphi_{2}$ and that $G$ has Lie rank at least 4 . In addition, assume $p \neq 3$ if $G={ }^{2} E_{6}(q)$ or $F_{4}(q), p \neq 5$ if $G={ }^{2} E_{6}(q)$ and $5 \mid q+1$, $p \neq 3,5$ if $G=E_{6}(q)$, and $p \neq 3,5,7$ if $G=E_{7}(q)$ or $E_{8}(q)$. Then $C_{G_{0}}^{0}\left(E_{0}\right)=G_{0}$.

Proof. Let $s$ be the positive root of highest height in $\Sigma$ and set $J_{1}=\left\langle U_{.}\right\rangle$. Choose conjugates $J_{1}, \ldots, J_{k}$ of $J_{1} \cong S L(2, q)$ with $k$ maximal such that $\left[J_{i}, J_{j}\right]=1$ for $i \neq j$. This can be done so that each $J_{i}$ is generated by a pair of opposite root subgroups of $G_{0}$ for roots in $\Sigma$. Then $H_{0}$ normalizes $J_{1} \cdots J_{k}$. One checks that $k=4$ if $G=F_{4}(q),{ }^{2} E_{6}(q), E_{6}(q), k=7$ if $G=E_{7}(q)$, and $k=8$ if $G=E_{8}(q)$. If $G=F_{4}(q), E_{7}(q)$, or $E_{8}(q)$, then $O\left(H_{0}\right) \leqslant J_{1} \cdots J_{k}$. In the other cases $J_{1} \cdots J_{k} O\left(H_{0}\right)=J_{1} \cdots J_{k} H_{1}$, where $H_{1}=O\left(H_{0}\right) \cap C\left(J_{1} \cdots J_{k}\right)$. So in all cases there is a subgroup $H_{1} \leqslant H_{0}$ with $I=J_{1} \cdots J_{k} O\left(H_{0}\right)=\left(J_{1} \cdots J_{k}\right) \times H_{1}$.

From order considerations, keeping in mind the prime restrictions, we see that $I$ contains a Sylow $p$-subgroup of $G_{0}$, so we may assume $E_{0} \leqslant I$. Considering the projection of $E_{0}$ into $J_{1} \cdots J_{k}$ we can apply (2.10) and get $I \leqslant C_{G_{0}}^{0}\left(E_{0}\right)$. In addition, 
for each $i=1, \ldots, k, E_{0}$ acts on $G_{i}=E\left(C_{G_{0}}\left(J_{i}\right)\right)$. Using $\$ 4$ and induction we have $G_{i} \leqslant C_{G_{0}}^{0}\left(E_{0}\right)$ for $i=1, \ldots, k$. An easy check gives $G_{0}=\left\langle G_{i}: i=1, \ldots, k\right\rangle$, proving the result.

(5.6) Suppose that $p$ is associated with $\varphi_{x}$ and one of the following holds: $G=E_{6}(q)$ and $x=3 ; G=E_{7}(q)$ and $x=6 ; G=E_{8}(q)$ and $x=3,4$ or 6 ; $G={ }^{2} E_{6}(q)$ and $x=6$. Then $D_{0}=G_{0}$.

Proof. First assume $x=3$. If $G=E_{6}(q)$, then from Table (3.3) we see that $G$ contains a central product $X_{1} X_{2} X_{3}$ of three copies of $S L(3, q)$. If $G=E_{8}(q)$, set $X_{4}=\left\langle U_{ \pm \alpha_{8}}, U_{ \pm s}\right\rangle$, where $s \in \Sigma^{+}$is the positive root of highest height. Then $X_{4} \cong S L(3, q)$ and $X_{4}$ is centralized by $\left\langle U_{ \pm \alpha_{1}}, \ldots, U_{ \pm \alpha_{6}}\right\rangle \cong E_{6}(q)$. So for $G=E_{6}(q)$ or $E_{8}(q), G_{0}$ contains a central product $X=X_{1} \cdots X_{k}$ of copies of $S L(3, q)$, where $k=3$ or 4 respectively. Now (5.2) implies that $X$ contains a Sylow $p$-subgroup of $G_{0}$, so we may assume $E_{0} \leqslant X$. A Sylow $p$-subgroup of $X$ is abelian of rank $k$, and there is a subgroup $E_{1} \cong Z_{p} \times Z_{p}$ such that $E_{0} \leqslant C\left(E_{1}\right)$ and $E_{1} \leqslant X_{1} X_{2}$. By (2.3) we have $G_{0}=C_{G_{0}}^{r}\left(E_{1}\right)$ (since $E_{1}$ centralizes a unipotent element of $X_{3}^{\#}$ ). By (2.5), (2.6)(ii), and either minimality of $G$ or the results of $\S 4$ we have $C_{G_{0}}^{r}\left(E_{1}\right) \leqslant C_{G_{0}}^{0}\left(E_{0}\right)$. This proves the result.

Essentially the same argument works for the other cases, but there are minor changes required. For $x=4, G=E_{8}(q)$ and Table (3.3) shows that $G_{0}$ contains a central product of two copies of $D_{4}(q)$. Then $G_{0}$ contains a central product, $X$, of four copies of $O^{-}(4, q)^{\prime} \cong L_{2}\left(q^{2}\right)$. From (5.2) one checks that a Sylow $p$-subgroup of $X$ is also one for $G_{0}$ unless $p=5$, in which case a Sylow 5-subgroup of $X$ is of index 5 in one for $G_{0}$. Suppose this occurs. The arguments used in the verification of Table (3.3) suffice to show that $S U\left(5, q^{2}\right) \leqslant E_{8}(q)$. Namely, use the groups $Y_{1}$ and $Y_{2}$ (of §3) together with the fundamental system

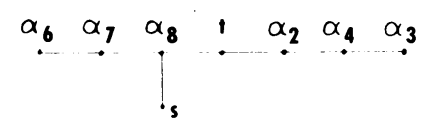

where $t=\alpha_{1}+\alpha_{2}+2 \alpha_{3}+3 \alpha_{4}+3 \alpha_{5}+2 \alpha_{6}+\alpha_{7}$. By orders we may take $E_{0} \leqslant S U\left(5, q^{2}\right)$ and it follows that $E_{0}$ is conjugate to a subgroup of $X$. So in all cases we may take $E_{0} \leqslant X$ and argue as before. The only difficulty is the possiblilty that $q=2, p=5$, and with $E_{1}$ as before, there is some $x \in E_{1}$ such that $C_{G_{0}}(x)$ contains a component of type $S z\left(2^{5}\right)$ or ${ }^{2} F_{4}(2)^{\prime}$. The former case is out by (2.5). The latter case is also impossible, since in order to get an ${ }^{2} F_{4}(2)^{\prime}$ component of $C_{G_{0}}(x), C_{\hat{G}}(x)$ would have to contain an $F_{4}(K)$ component. But this cannot happen since the components of $C_{\hat{G}}(x)$ all have root systems being a subset of the root system of type $E_{8}$.

For $x=6$ again use Table (3.3) to conclude that ${ }^{2} E_{6}(q)$ contains a central extension of $U_{3}(q) \times U_{3}(q) \times U_{3}(q), E_{7}(q)$ a central extension of ${ }^{3} D_{4}(q) \times L_{2}\left(q^{3}\right)$, and $E_{8}(q)$ a central extension of ${ }^{3} D_{4}(q) \times{ }^{3} D_{4}(q)$. We may assume that $E_{0}$ is contained in the appropriate central product (again by (5.2)). Now ${ }^{3} D_{4}(q)$ contains a central extension of $L_{2}(q) \times L_{2}\left(q^{3}\right)$. So if $m_{p}\left({ }^{3} D_{4}(q)\right)=1$, this would force $E_{0}$ to 
centralize a nontrivial $r$-subgroup, whence (2.3) gives the result. So we may assume $m_{p}\left({ }^{3} D_{4}(q)\right)=2$ and choose a suitable $E_{1}$, as before.

In the next two results the contradiction is reached by combining the results of Table (3.3), (2.8), and the centralizer information in $\hat{G}$.

(5.7) Let $p$ be associated with $\varphi_{x}$. In each of the following cases we have $C_{\left(G_{0}\right)}^{0}\left(E_{0}\right)=G_{0}: G=F_{4}(q)$ and $x=4 ; G={ }^{2} E_{6}(q)$ and $x=3,4 ; G=E_{6}(q)$ and $x=6 ; G={ }^{3} D_{4}(q)$ and $x=1,2$, but $p \neq 3 ; G=E_{8}(q)$ and $x=8$.

Proof. Suppose false. First we point out the existence of a large subgroup, $X$, of $G$. These are presented as triples $\left(G, \varphi_{x}, X / Z(X)\right)$ and are as follows: (i) $\left(F_{4}(q), \varphi_{4}, P S O(9, q)^{\prime}\right)$; (ii) $\left({ }^{2} E_{6}(q), \varphi_{3}, F_{4}(q)\right)$; (iii) $\left({ }^{2} E_{6}(q), \varphi_{4}, P S O^{-}(10, q)^{\prime}\right)$; (iv) $\left(E_{6}(q), \varphi_{6}, F_{4}(q)\right)$; (v) $\left({ }^{3} D_{4}(q), \varphi_{1}\right.$ or $\left.\varphi_{2}, G_{2}(q)\right)$; (vi) $\left(E_{8}(q), \varphi_{8}, P S^{+}(16, q)^{\prime}\right)$. In cases (i), (iii), and (vi) the existence of $X$ follows from consideration of the extended Dynkin diagram of $G$. In the other cases $X$ is obtained from the centralizer of a graph automorphism of $G_{0}$. In all cases $X$ is generated by long root subgroups of $G_{0}$. Moreover from (5.2) we check that $X$ contains a Sylow $p$-subgroup of $G_{0}$, so we take $E_{0} \leqslant X$. We note that $m_{p}\left(E_{0}\right)=2$. By minimality of $G$ and by results of $\S 4$ we have $X \leqslant C_{G_{0}}^{0}\left(E_{0}\right)$. Let $D_{0}=C_{G_{0}}^{0}\left(E_{0}\right)$.

Let $V$ be a long root subgroup of $G_{0}$ with $V \leqslant X \leqslant D_{0}$. Set $I=\left\langle V^{G_{0}} \cap D_{0}\right\rangle$. Then $D_{0} \leqslant N_{G_{0}}(I) \geqslant X$ and the structure of $I$ is given in (2.8). We may assume (2.8)(i) does not hold. Let $\bar{I}=I$ if $q>3$, and $\bar{I}=I / O_{3}(I)$ or $I / O_{2}(I)$ if $q=2$ or 3 , respectively. Write $\bar{I}=\bar{I}_{1} \cdots \bar{I}_{k}$, a central product as given in (2.8). As $X=\left\langle V^{(i} \cap X\right\rangle$ we may take $X \leqslant I_{1}$. If $k>1$, then some $r$-local subgroup of $G_{0}$ involves $X$, against $h\left(r, E_{0}\right)=1$. So $i=1$ and $I=I_{1}$. As $X \leqslant I$ it follows that $\bar{I}$ is a central extension of a group of Lie type defined over $\mathbf{F}_{q}$. Comparing orders we have $\bar{X}=\bar{I}$. In this comparison use the fact that $|\bar{I}|_{r} \leqslant\left|G_{0}\right|_{r}$ and the existence of primitive divisors. (A prime divisor $s$ of $r^{a}-1$ is a primitive divisor if $s \nmid r^{b}-1$ for $b<a$. Such divisors exist except when $r^{a}=2^{6}$ or $r^{a}=r^{2}$ with $r$ a Mersenne prime.)

Now $D_{0} \leqslant N(I)$, so $D_{0}$ acts on $\bar{X}=\bar{I}$, and as no parabolic subgroup of $G_{0}$ contains a section isomorphic to $\bar{X} / Z(\bar{X})$ we conclude that $\bar{X}=\left(O^{r^{\prime}}\left(\bar{D}_{0}^{(\infty)}\right)\right)$. We will show this to be impossible. Say $G \cong F_{4}(q)$ with $q$ even. Then the Frattini argument shows that $E_{0}$ is normalized by an element of $\operatorname{Aut}\left(G_{0}\right)$ lying in the coset of a graph automorphism. But no such automorphism can normalize $D_{0}$. Say $G \cong F_{4}(q)$ with $q$ odd. Here we use the embedding of $S O^{-}(4, q) \times S O^{-}(4, q) \leqslant P S O(9, q)^{\prime}$ to conclude that $E_{0}$ centralizes a klein group $C \leqslant G_{0}$ with $C$ contained in a parabolic subgroup of $G_{0}$. Then (2.3) gives $C_{G_{0}}^{0}(C)=G_{0}$. Now use induction and the results of $\S 4$ to conclude $D_{0}=G_{0}$. For $G={ }^{2} E_{6}(q)$ we have $E_{0} \leqslant J$ where $J \cong L_{3}(q) \times L_{3}\left(q^{2}\right)$ or $F_{4}(q)$, according to whether we are in case (ii) or (iii). We then have $J \leqslant I$, and order considerations contradict $\bar{X}=\left(O^{r^{\prime}}\left(\bar{D}_{0}\right)\right)^{(\infty)}$.

If $G \cong E_{6}(q)$, use Table (3.3) to get a subgroup $J$ such that $J / Z(J) \cong L_{3}\left(q^{2}\right) \times U_{3}(q)$. By orders we may take $E_{0} \leqslant J$ and thus $J \leqslant D_{0}$. But then $L_{3}\left(q^{2}\right) \times U_{3}(q) \leqslant \bar{X}=F_{4}(q)$, whereas a consideration of the parabolics of $F_{4}(q)$ shows this to be impossible. Similarly, if $G \cong{ }^{3} D_{4}(q)$, then $G_{0}$ contains a subgroup isomorphic to $S L_{2}(q) \circ S L_{2}\left(q^{3}\right)$. But then $E_{0}$ is in such a subgroup and $\overline{D_{0}}$ contains $L_{2}(q) \times L_{2}\left(q^{3}\right)$. However $G_{2}(q)$ contains no such subgroup, so $\left(O^{r^{\prime}}\left(\bar{D}_{0}^{(\infty)}\right)\right) \neq \bar{X}$. 
Finally, suppose $G=E_{8}(q), p$ is associated with $q^{8}-1$ and $\bar{X} \cong \operatorname{PSO}^{+}(16, q)^{\prime}$. Let $M$ be the natural module for $Y=O^{+}(16, q)$, choose $e \in E_{0}^{\#}$, and regard $e \in Y$. Then $e$ acts on $M$ and we set $Y_{0}=O^{r^{\prime}}\left(C_{Y}(e)\right)$. Write $M=M_{1} \oplus M_{2}$, a sum of $\langle e\rangle$-invariant 8 -spaces of $M$. One checks that $Y_{0}=1, O^{-}(8, q)^{\prime}$, or $S L\left(2, q^{4}\right)$ depending on the action of $e$ on $M_{1}$ and $M_{2}$. However, by (3.3), $G_{0}$ contains a central extension of $O^{-}(12, q)^{\prime} \times U_{3}(q)$. So $G_{0}$ contains a central extension of $O^{-}(8, q)^{\prime} \times O^{+}(4, q) \times U_{3}(q)$, and some element, $e$, of $E_{0}-Z\left(G_{0}\right)$ centralizes a subgroup isomorphic to $\mathrm{SO}^{+}(4, q) \times U_{3}(q)$. Moreover, each of the factors of the subgroup is generated by conjugates of $V$, so $C_{I}(e)$ contains $S O^{+}(4, q) \times U_{3}(q)$, contradicting $\bar{X}=\bar{I}$. This completes the proof of (5.7).

(5.8) Let $p$ be associated with $\varphi_{x}$. Then none of the following occur: $G=G_{2}(q)$, $p>3$, and $x=1,2 ; G=E_{8}(q)$ and $x=5,10,12 ; G=F_{4}(q)$, and $x=3,6$.

Proof. As in the proof of (5.7) we produce a certain subgroup $X$ of $G$. However this time $X$ will be a central product of two quasisimple Chevalley groups. The triples $\left(G, \varphi_{x}, X / Z(X)\right)$ are as follows: $\left(F_{4}(q), \varphi_{3}, L_{3}(q) \times L_{3}(q)\right)$, $\left(F_{4}(q), \varphi_{6}, U_{3}(q) \times U_{3}(q)\right),\left(E_{8}(q), \varphi_{5}, L_{5}(q) \times L_{5}(q)\right),\left(E_{8}(q), \varphi_{10}, U_{5}(q) \times U_{5}(q)\right)$, $\left(E_{8}(q), \varphi_{12},{ }^{3} D_{4}(q) \times{ }^{3} D_{4}(q)\right),\left(G_{2}(q), \varphi_{1}\right.$ or $\left.\varphi_{2}, L_{2}(q) \times L_{2}(q)\right)$. The existence of $X$ follows from Table (3.3) in all but the first and last cases. In the first case let $X=X_{1} X_{2}$, where $X_{1}=\left\langle U_{ \pm \alpha_{3}}, U_{ \pm \alpha_{4}}\right\rangle$ and $X_{2}=\left\langle U_{ \pm \alpha_{1}}, U_{ \pm s}\right\rangle$. In the last case let $X=X_{1} X_{2}$, where $X_{1}=\left\langle U_{ \pm \alpha_{2}}\right\rangle$ and $X_{2}=\left\langle U_{ \pm s}\right\rangle$. (In each case $s$ denotes the positive root of highest height in $\Sigma$.) Using Table (5.2) we check that $X$ contains a Sylow $p$-subgroup of $G_{0}$, so we may take $E_{0} \leqslant X$.

First we claim that each component of $X$ has $p$-rank 1 , so that $E_{0} \cong Z_{p} \times Z_{p}$. This is clear from the theory of linear groups except for the case where $X={ }^{3} D_{4}(q) \times{ }^{3} D_{4}(q)$. Suppose $m_{p}\left({ }^{3} D_{4}(q)\right)>1$. Here the argument of (5.6) applies. Namely, $E_{0} \leqslant C\left(E_{1}\right)$ with $E_{1} \cong Z_{p} \times Z_{p}$ and $E_{1}$ in one of the components of $X$. From here induction, the main results of $\S 4$, and (2.3) give a contradiction to $C_{G_{0}}^{0}\left(E_{0}\right)<G_{0}$. This proves the claim and we write $E=E_{1} \times E_{2}$, where $E_{i}=E \cap X_{i}$ and $X_{1}, X_{2}$ are the components of $X$. Clearly $X \leqslant C_{G_{0}}^{0}\left(E_{0}\right)$.

Next we set $I=\left\langle V^{G_{0}} \cap D_{0}\right\rangle$ for $V$ a long root subgroup of $G_{0}$. We will determine the structure of $I$ as in (5.7). We have $X \leqslant D_{0} \leqslant N_{G_{0}}(I)$ and either by (3.4) or by construction, at least one of the components of $X$ is generated by conjugates of $V$. We use (2.8) to obtain the structure of $I$. Set $\bar{I}=I$ if $q>3, \bar{I}=I / O_{2}(I)$ if $q=3$, and $\bar{I}=I / O_{3}(I)$ if $q=2$. Now $X \leqslant D_{0} \leqslant N_{G_{0}}(I)$ and at least one of the components of $X$ is actually contained in $I$. By (2.3) and (2.8) the structure of $\bar{I} / Z(\bar{I})$ is known and $\bar{I}=\bar{I}_{1} \cdots \bar{I}_{k}$, a central product, with $\bar{I}_{i}=\left\langle\bar{V}_{i} \bar{I}\right\rangle$ for some $V_{i} \in V^{G}$ and $\bar{I}_{i}$ is either a Chevalley group over $\mathbf{F}_{q}$, or $q=2$ and $E\left(\bar{I}_{i}\right)$ is described in (2.8)(iv). Since $m_{p}\left(E_{0}\right)=m_{p}\left(G_{0}\right)=2$ and $\boldsymbol{U}\left(r, E_{0}\right)=\{1\}$, we must have $X \leqslant N\left(\bar{I}_{i}\right)$ for $i=1, \ldots, k$. As $X=X^{\prime}, X$ induces a group of inner automorphisms on each $\bar{I}_{i}$, and since $\boldsymbol{U}\left(r, E_{0}\right)=\{1\}$ (by (2.3)) we conclude that $k \leqslant 2$. Indeed, if $k>2$, then there exists $i \in\{1, \ldots, k\}$ such that $p \nmid\left|\bar{I}_{i}\right|$, and for this $i,\left[X, \bar{I}_{i}\right]=1$. Now let $J=I X$ and $\bar{J}=I X, I X / O_{2}(I)$, or $I X / O_{3}(I)$, depending on whether $q>3, q=3$, or $q=2$. Then either $\bar{J} \neq \bar{I}$, or $\bar{J}=\bar{I} \bar{L}$ where $[\bar{I}, \bar{L}]=1$. In the second case we must have $k=m_{p}(\bar{I})=m_{p}(\bar{L})=1$. This can be seen by using the facts that $m_{p}\left(G_{0}\right)=2$ and $\boldsymbol{U}\left(r, E_{0}\right)=\{1\}$. Consequently $\bar{E}_{0} \cap \bar{L} \neq 1$, and we conclude that $\bar{L}$ is the image of 
one of the components of $X$. Write $\bar{J}=\bar{J}_{1} \bar{J}_{2}$ a central product, with $E\left(\bar{J}_{1}\right)$ quasisimple, and $E\left(\bar{J}_{2}\right)$ quasisimple or $\bar{J}_{2}=1$. Also we have $\bar{X}=\left(\bar{X} \cap \bar{J}_{1}\right)\left(\bar{X} \cap \bar{J}_{2}\right)$ and $X=X^{\prime}$. Thus $\bar{I}^{\prime}$ contains root elements. If $q=2$, this rules out all exceptional cases of (2.8)(iv) other than the Fischer groups $F_{22}, F_{23}, F_{24}$. Each of $\left|F_{23}\right|$ and $\left|F_{24}\right|$ is divisible by 23 , whereas $23 \nmid\left|G_{0}\right|$. So these cases are out. If $\overline{J_{i}} / Z\left(\bar{J}_{i}\right) \cong F_{22}$, then order arguments force $G_{0}=E_{8}(2)$ and $\bar{J} \bar{X} \cong \bar{X}_{1} \times F_{22}$. By [6] some parabolic of $G_{0}$ contains a Sylow $r$-subgroup of $\bar{X}_{1}$ in its unipotent radical and $F_{22}$ in its Levi factor. Consideration of the nilpotence class of the Sylow $r$-subgroup of $X_{1}$ and order considerations lead to a contradiction. Therefore, $\bar{J}=\bar{J}_{1} \bar{J}_{2}$ with each of $\bar{J}_{1}, \bar{J}_{2}$ a central extension of a Chevalley group over $\mathbf{F}_{q}$.

We claim that $\bar{J}=\bar{X}$ and we indicate by example how this is proved. Say $G=E_{8}(q)$ and $p$ is associated with $\varphi_{5}$. Then $X / Z(X) \cong L_{5}(q) \times L_{5}(q)$. Suppose that $\bar{X} \leqslant \bar{J}_{1}$. Checking orders of Chevalley groups defined over $\mathbf{F}_{q}$ we conclude that $\bar{J}_{1}$ must be an extension of $L_{10}(q)$. This implies that some $p$-local subgroup of $G$, and hence some parabolic subgroup of $G$, involves $L_{9}(q)$. The parabolic subgroup is necessarily the maximal parabolic of $E_{8}(q)$ that involves $E_{7}(q)$. Repeating this we see that some proper parabolic subgroup of $E_{7}(q)$ must involve $L_{8}(q)$. This is impossible (compare orders). So we may assume $\bar{X}_{1} \leqslant \bar{J}_{1}$ and $\bar{X}_{2} \leqslant \bar{J}_{2}$. Say $\bar{J}_{i}>\bar{X}_{i}$. Considering possible choices for $\bar{J}_{i}$ we can argue as above. For example if $\bar{J}_{i}$ involves $L_{6}(q)$, then some proper parabolic subgroup of $G_{0}$ involves $L_{5}(q) \times L_{5}(q)$ and we argue as above to get a contradiction. Thus $\bar{J}=\bar{X}$ as claimed. The other cases are similar, but there is one troublesome point when $G=E_{8}(q)$ and $p$ is associated with $\varphi_{10}$. Namely, we must rule out the case $\bar{J} / Z(\bar{J}) \cong P S U(10, q)$. Suppose that this occurs. Then $\bar{J}$ has a parabolic subgroup involving $L_{5}\left(q^{2}\right)$ and containing an element $e \in E^{\#}$. It follows that $e$ is in a parabolic subgroup, $P$, of $E_{8}(q)$ involving either $E_{7}(q)$ or $D_{7}(q)$. We claim that $C_{G_{0}}(e)$ contains a conjugate of $V$. In the $E_{7}(q)$ case this follows from the fact that $Z\left(O^{r^{\prime}}(P)\right)$ is a long root subgroup. For the $D_{7}(q)$ case use (17.14)(ii) of [3] to see that the Levi factor of $P$ acts on $O_{r}(P)^{\prime}$ as on the natural module for $O^{+}(14, q)^{\prime}$ and root groups correspond to singular 1-spaces. Considering the action of $e$ on the usual module, we have the claim. On the other hand $C_{J}(e)$ contains no conjugate of $V$, and this contradicts the definition of $J$. So in all cases we have the claim that $\bar{J}=\bar{X}$.

By (2.7)(iii) and (2.11) we may take $E_{0} \leqslant \hat{H}$ and find $e \in E_{0}-\left(E_{1} \cup E_{2}\right)$ such that $C_{\hat{U}}(e) \neq 1$. It follows that $L_{0}=O^{r^{\prime}}\left(C_{G_{0}}(e)\right)$ is a nontrivial central product of Chevalley groups defined over extension fields of $\mathbf{F}_{q}$ (see (2.6)). Since $\boldsymbol{h}\left(r, E_{0}\right)=\{1\}$, $E_{0} \cap E\left(L_{0}\right) \neq 1$. In particular $E\left(L_{0}\right) \neq 1$ and $E\left(L_{0}\right)$ induces a group of inner automorphisms on $\bar{X}$. But $\boldsymbol{И}\left(r, E_{0}\right)=\{1\}$ implies $E\left(L_{0}\right) \leqslant J$ and so $\overline{E\left(L_{0}\right)} \leqslant \bar{X}_{1} \bar{X}_{2}$. However $C(e) \cap \bar{X}_{1} \bar{X}_{2}$ is an $r^{\prime}$-group, so this is a contradiction.

At this point we have dealt with all cases except those for which either $p$ is "small" or $G_{0}$ has Lie rank 2 .

(5.9) Let $G=F_{4}(q)$ or ${ }^{2} E_{6}(q)$. If $G={ }^{2} E_{6}(q)$ and $p=3$, then suppose $p \mid q-1$. One of the following holds:

(i) $D_{0}=G_{0}$.

(ii) $p=3, G_{0}=F_{4}(2)$ or $F_{4}(4)$, and for suitable choice of $E_{0}, C_{G_{0}}^{0}\left(E_{0}\right) \leqslant N_{G_{0}}\left(E_{0}\right)$. 
Proof. By Table (5.2) and the results of (5.3)-(5.8), the only cases to consider are $p=5 \mid q+1$ with $G={ }^{2} E_{6}(q)$ and $p=3 \mid q \pm 1$. First consider the case $p=5$. Then $G={ }^{2} E_{6}(q)$ and from the extended Dynkin diagram of $G$ we find a subgroup, $X$, of $G$ with $X$ a central extension of $\operatorname{PSO}^{-}(10, q)^{\prime}$ and $X$ generated by long root subgroups. Considering the action of $H$ on $X$ we then find a subgroup $H_{1} \leqslant X$ with $\left[H_{1}, X\right]=1$ and $H_{1}$ of order divisible by $(q+1) /(3, q+1)$. From order considerations we may take $E_{0} \leqslant H_{1} X$. One then argues that $E_{0} \leqslant O_{p}\left(H_{1}\right) \times F$, where $F$ is the direct product of 5 copies of $O_{p}\left(Z_{q+1}\right)$ and $F$ acts on the usual module for $O^{-}(10, q)$ respecting a decomposition into five pairwise orthogonal, anisotropic 2-spaces. Hence there is a subgroup $E_{1} \leqslant F$ with $E_{0} \leqslant C\left(E_{1}\right)$, $E_{1} \cong Z_{p} \times Z_{p}$, and $E_{1}$ centralizes a proper $r$-subgroup of $X$. So $C_{G_{0}}^{r}\left(E_{1}\right)=G_{0}$ by (2.3). Then minimality of $G$ together with (2.16) and the results of $\$ 4$ imply $G_{0}=C_{G_{0}}^{r}\left(E_{1}\right) \leqslant D_{0}$. So from now on we may assume $p=3$.

Suppose $G=F_{4}(q)$. From Table (3.3) we have a subgroup $L \leqslant G_{0}$ such that there exists $L_{0}<L$ with $\left|L: L_{0}\right|=3$ and $L_{0}=L_{1} L_{2}$, the central product of two copies of $S L(3, q)$ or $S U(3, q)$, according to $3 \mid q-1$ or $3 \mid q+1$. If $3 \mid q-1$, then we may take $L_{2}=\left\langle U_{ \pm \alpha_{3}}, U_{ \pm \alpha_{4}}\right\rangle$ and $L_{1}=\left\langle U_{ \pm s}, U_{ \pm \alpha_{1}}\right\rangle$ where $s$ is the positive root of highest height. Now suppose $G={ }^{2} E_{6}(q)$ and $3 \mid q-1$. We regard $F_{4}(q) \leqslant{ }^{2} E_{6}(q)$ and write ${ }^{2} E_{6}(q)=\left\langle U_{ \pm \alpha_{1}}, U_{ \pm \alpha_{2}}, \hat{U}_{ \pm \alpha_{3}}, \hat{U}_{ \pm \alpha_{4}}\right\rangle$, where $U_{\alpha_{3}} \leqslant \hat{U}_{\alpha_{3}}, U_{\alpha_{4}} \leqslant \hat{U}_{\alpha_{4}}$, etc. Here we have a subgroup $\hat{L}>L$ such that $\hat{L}$ contains $\hat{L}_{0}=L_{1} \hat{L}_{2}$ as a normal subgroup of index 3 , where $\hat{L}_{2}=\left\langle\hat{U}_{ \pm \alpha_{3}}, \hat{U}_{ \pm \alpha_{4}}\right\rangle \cong S L\left(3, q^{2}\right)$. Then $L_{0}<\hat{L}_{0}$, $L_{2}=\hat{L}_{2} \cap L$, and $\left[L_{1}, \hat{L}_{2}\right]=1$. Let $\langle z\rangle=Z(L)=Z(\hat{L})$.

Suppose $q \neq 2,4$ and $G_{0}=F_{4}(q)$. We may assume that $z \in E_{0}$ (use (2.9)(a)). We claim that there exist 3 -elements $x_{1} \in L_{1}$ and $y_{1} \in L_{2}$ such that $\left|x_{1} y_{1}\right|=3$, $x_{1} y_{1} \in L_{0}-Z\left(L_{0}\right)$, and $x_{1} y_{1} \in C\left(E_{0}\right)$. If $E_{0} \cap L_{0} \neq Z\left(L_{0}\right)$, then just choose $x_{1} y_{1} \in E_{0}-\langle z\rangle$. Suppose $E_{0} \cap L_{0}=\langle z\rangle$. Then $E_{0}=\langle z\rangle \times\left\langle z^{\prime}\right\rangle$ for $z^{\prime} \in L-L_{0}$. Choose $x_{1} \in L_{1}, y_{1} \in L_{2}$ each of order 3 such that $z^{\prime}$ centralizes $x_{1}$ and $y_{1}$ mod $(\langle z\rangle)$. Then $z^{\prime}$ centralizes one of $x_{1}, y_{1}, x_{1} y_{1}$, or $x_{1} y_{1}^{-1}$ and the claim follows. If $E_{0} \leqslant C\left(x_{1}\right)$ or if $E_{0} \leqslant C\left(y_{1}\right)$, then set $E_{1}=\left\langle x_{1}, z\right\rangle$, respectively $\left\langle y_{1}, z\right\rangle$. Then $E_{1}$ centralizes a component of $L_{0}$ so $C_{G_{0}}^{r}\left(E_{1}\right)=G_{0}$ by (2.3). By induction, (2.16), (2.6)(ii), (2.5), and $\S 4$ we have $D_{0}=G_{0}$. So suppose neither $x_{1}$ nor $y_{1}$ is centralized by $E_{0}$.

Let $E_{1}=\left\langle z, x_{1} y_{1}\right\rangle$. Then $E_{0} \leqslant C\left(E_{1}\right)$ and if $E_{1}$ normalizes a proper $r$-subgroup of $G_{0}$, we can argue as above. Suppose this is not the case. Then $3 \mid q+1$, $L_{1} \cong L_{2} \cong S U(3, q)$ and relabeling, if necessary, $x_{1}$ is in a conjugate, $C$, of $\left\langle U_{ \pm s}\right\rangle$, where $s$ is the root of highest height and where $C \leqslant L_{1}$. Then $E_{1}<N(C)^{\prime}=C \times I$, where $I \cong S p(6, q)$. Considering elementary abelian 3-subgroups of $S p(6, q)$ we argue that there exist conjugates $C_{1}, C_{2}$, and $C_{3}$ of $C$ such that $E_{1}<C \times C_{1} \times C_{2} \times C_{3}$. We claim that $I \leqslant C_{G_{0}}^{r}\left(E_{1}\right)$. This will follow from (3.1) once we show that the copies of $\operatorname{Sp}(4, q)$ in $I$ corresponding to $C_{1} \times C_{2}$ and $C_{2} \times C_{3}$ are in $C_{G_{0}}^{r}\left(E_{1}\right)$. Say, for example, $I_{0}$ is the copy of $\operatorname{Sp}(4, q)$ with $C_{1} \times C_{2} \leqslant I_{0}$ and $\left[I_{0}, C_{3}\right]=1$. Then $C_{1} \times C_{2} \leqslant C_{I_{0}}^{r}\left(E_{1}\right)$ and by (3.2) either $I_{0}=C_{I_{0}}^{r}\left(E_{1}\right)$ or $C_{I_{0}}^{r}\left(E_{1}\right)$ normalizes $C_{1} \times C_{2}$. A direct check shows the latter to be impossible. Thus $I_{0} \leqslant C_{G_{0}}^{r}\left(E_{1}\right)$ and we conclude that $I \leqslant C_{G_{0}}^{r}\left(E_{1}\right)$. We may now assume that 
$C_{3}=\left\langle U_{ \pm \alpha}\right\rangle$, for $\alpha=\alpha_{2}+2 \alpha_{3}+2 \alpha_{4}$. By symmetry $\tilde{I}=E\left(C_{G_{0}}\left(C_{3}\right)\right) \leqslant C_{G_{0}}^{r}\left(E_{1}\right)$. A check with root systems shows that $\langle I, \tilde{I}\rangle=G_{0}$, so $G_{0}=C_{G_{0}}^{r}\left(E_{1}\right)$ and we argue as before that $G_{0}=D_{0}$. Now suppose $G={ }^{2} E_{6}(q)$ with $3 \mid q-1$ and $q>4$. Then we may take $E_{0}<L<\hat{L}$, so by the above minimality of $G$ and $\S 4$ we conclude $C_{G_{0}}^{0}\left(E_{0}\right) \geqslant\left\langle\hat{L}, F_{4}(q)\right\rangle=G_{0}$. So for these cases (i) holds.

Next suppose that $q=2$ and $G=F_{4}(2)$. We will exhibit an elementary abelian subgroup, $F$, for which generation fails. Write $O_{3}\left(L_{1}\right)=\left\langle x_{1}, x_{2}\right\rangle$ and $O_{3}\left(L_{2}\right)=\left\langle y_{1}, y_{2}\right\rangle$, so that $\left\langle x_{1}, x_{2}\right\rangle \cong\left\langle y_{1}, y_{2}\right\rangle$ is extraspecial of order $3^{3}$. Then $L_{i}=O_{3}\left(L_{i}\right) Q_{i}$ where $Q_{i} \cong Q_{8}$ for $i=1,2$. Finally, $L=L_{0}\langle e\rangle$, where $|e|=3$. To see that $e$ exists just observe that $L$ contains a Sylow 3-subgroup of $F_{4}(2)$ and that $F_{4}(2)$ contains a direct product of four copies of $S_{3}$ (each generated by long root subgroups). So $F_{4}(2)$ has 3 -rank at least 4 , whereas $L_{0}$ has 3 -rank 3 . We may assume that $e \in C\left(x_{1}\right) \cap C\left(y_{1}\right)$, so setting $Z\left(L_{0}\right)=\langle z\rangle$ we let $F=\left\langle z, e, x_{1}, y_{1}\right\rangle$. By (3.4) and the structure of $S U(3,2)$ we may assume that the involution in $Q_{1}$ is a root involution of $G_{0}$, and that $x_{1}$ is a product of two root involutions. This is true for each $x \in\left\langle x_{1}, z\right\rangle-\langle z\rangle$. So for each such $x, C_{G_{0}}(x)=\langle x\rangle \times S p(6,2)$. Let $F_{1}$ be maximal in $F$. If $x \in F_{1}$ for $x \in\left\langle x_{1}, z\right\rangle-\langle z\rangle$, then $C\left(F_{1}\right) \leqslant\langle x\rangle \times S p(6,2)$ and we have $C_{G_{0}}\left(F_{1}\right) \leqslant N(F)$ (see (4.1) and the proof of (4.3)). From the work of Burgoyne [7] we have $L=C_{G_{0}}(z)$ and we can argue that $L$ is normalized by a graph automorphism of $G_{0}$, which necessarily interchanges $L_{1}$ and $L_{2}$. Consequently, if $F_{1}$ contains an element of $\left\langle y_{1}, z\right\rangle-\langle z\rangle$ we again get $C_{G_{0}}\left(F_{1}\right) \leqslant N(F)$. So we may assume that $F_{1}$ contains $z$ and an element $g=x_{1}^{i} y_{1}^{j}$ for $i= \pm 1, j= \pm 1$. Then $C_{G_{0}}\left(F_{1}\right) \leqslant C_{G_{0}}(z) \cap C_{G_{0}}(g)=C_{L}(g)$. But $C_{L}(g) \leqslant O_{3}(L)\langle e\rangle$ and it is easy to see that $C_{L}(g) \leqslant N(F)$. So (ii) holds.

Suppose $q=4$ and $G=F_{4}(4)$ or ${ }^{2} E_{6}(4)$. Write $L_{0}=L_{1} L_{2}$ with $L_{1} \cong L_{2} \cong S L(3,4)$ and $\hat{L}_{0} \cong L_{1} \hat{L}_{2}$ with $L_{2} \leqslant \hat{L}_{2} \cong S L(3,16)$. Let $J \in \operatorname{Syl}_{3}(L)$ and set $J_{i}=J \cap L_{i}$ for $i=1,2$. Then $J_{1} \cong J_{2}$ is extraspecial of order $3^{3}$ and as $J$ has 3-rank 4 (as for $F_{4}(2)$ ) we may write $J=J_{1} J_{2}\langle e\rangle$ with $\langle e\rangle=3$. Set $\langle z\rangle=Z(J)=Z\left(J_{1}\right)=Z\left(J_{2}\right)$ and note that $J \in \operatorname{Syl}_{3}(\hat{L})$. By (2.9) we may assume $z \in E_{0}$. Suppose that $a \in E_{0}$ with $a \in J_{i}-\langle z\rangle$. Then $E_{0} \leqslant C_{G_{0}}(a)$. Now $a \in J$ where $J$ is generated by two opposite root subgroups of $G$, so $C_{G_{0}}(a)=\langle a\rangle \times I$ where $I \cong S p(6, q)$ if $G=F_{4}(q)$ and $I \cong S U(6,4)$ or $O^{-}(8,4)^{\prime}$ if $G={ }^{2} E_{6}(4)$. Now argue that $E_{0}$ normalizes an $r$-subgroup of $I$ (one can argue as in (4.2)). So in this case (i) holds by (2.3). Now assume that $E_{0}$ contains no such element, $a$, or any conjugate of such an element.

By Burgoyne [7] each $a \in E_{0}^{\#}$ is conjugate to $z$ and $C_{G_{0}}(a) \cong C_{G_{0}}(z)=L$ (respectively $\hat{L}$ ). For $F_{4}(4)$ we will produce a subgroup $E_{0}$ for which (ii) holds, and for ${ }^{2} E_{6}(4)$ we will show that (i) holds. First consider $G=F_{4}(4)$. As before write $J_{1}=\left\langle x_{1}, x_{2}\right\rangle$ and $J_{2}=\left\langle y_{1}, y_{2}\right\rangle$, where $\left[x_{1}, x_{2}\right]=z$ and $\left[y_{1}, y_{2}\right]=z^{-1}$. Set $E_{0}=\left\langle z, x_{1} y_{1}, x_{2} y_{2}\right\rangle$. If $e \in E_{0}-\langle z\rangle$, then $C_{G_{0}}(e) \cap C_{G_{0}}(z)=C_{L}(e)$ is solvable. By Burgoyne [7] it follows that $z \sim e$. So $E_{0}^{\#}$ is fused in $G_{0}$.

From the construction of $L$ one can show that there exists $e \in J-J_{1} J_{2}$ such that $x_{1}^{e}=x_{1}, y_{1}^{e}=y_{1}, x_{2}^{e}=x_{2} x_{1}$, and $y_{2}^{e}=y_{1} y_{2}$. Let $F$ be a hyperplane of $E_{0}$ with $z \in F$. As $N_{L}\left(E_{0}\right)$ is transitive on $\left(E_{0} /\langle z\rangle\right)^{\#}$, in order to show $C_{G_{0}}(F) \leqslant N_{G_{0}}\left(E_{0}\right)$ it will be enough to show $C_{G_{0}}\left(\left\langle z, x_{1} y_{1}\right\rangle\right) \leqslant N_{G_{0}}\left(E_{0}\right)$. But

$$
C_{G_{0}}\left(\left\langle z, x_{1} y_{1}\right\rangle\right)=C_{L}\left(x_{1} y_{1}\right)=\left\langle x_{1}, x_{1} y_{1}, z, x_{2} y_{2}, e\right\rangle \leqslant N_{G_{0}}\left(E_{0}\right),
$$


as needed. Now let $F$ be an arbitrary hyperplane in $E_{0}$. Say $z^{g} \in F$. Then $E_{0} \leqslant L^{g}$. If $F \leqslant L_{0}^{g}$, then $F$ is $L_{0}^{g}$-conjugate to $\left\langle z^{g},\left(x_{1} y_{1}\right)^{g}\right\rangle$, so $C_{G_{0}}(F)$ is $L_{0}^{g}$-conjugate to $C_{L}\left(x_{1} y_{1}\right)^{g}$, and as $C_{L}\left(x_{1} y_{1}\right)^{g} /\left\langle z^{g},\left(x_{1} y_{1}\right)^{g}\right\rangle$ is abelian, we have $C_{G_{0}}(F) \leqslant N\left(E_{0}\right)$.

We claim that $N_{G_{0}}\left(E_{0}\right)$ is transitive on $E_{0}^{\#}$. We already have $N_{L_{0}}\left(E_{0}\right)$ transitive on $\left(E_{0} /\langle z\rangle\right)^{\#}$ and it follows that $E_{0}-\langle z\rangle$ is fused in $N_{G_{0}}\left(E_{0}\right)$.

It will suffice to show that $U^{\#}$ is fused in $N_{G_{0}}\left(E_{0}\right)$, where $U=\left\langle z, x_{1} y_{1}\right\rangle$. Let $P=\left\langle z, x_{1}, y_{1}, x_{2}, y_{2}, e\right\rangle \in \operatorname{Syl}_{3}(G)$. One checks that $U \unlhd P, C_{P}(U) / U$ is elementary of order $3^{3}$, and $U=C_{P}(U)^{\prime}$. Let $z^{g} \in U-\langle z\rangle$. Then $C_{P}(U)$ has index 3 in a Sylow 3-subgroup of $C_{G}\left(z^{g}\right)$ and it follows that $N_{G}(U) / C_{G}(U)$ contains $S L(2,3)$. Also, $C_{G}(U)=C_{L}(U)=C_{P}(U)$. If $w_{0}$ is the long word in $W$, then $w_{0}$ induces a graph automorphism on $L_{1}$ and on $L_{2}$, so notation may be chosen so that $w_{0}$ inverts $U, w_{0} \in N\left(E_{0}\right)$, and $w_{0}$ centralizes $E_{0} / U$. It follows that $E_{0} / U=C\left(w_{0}\right) \cap C_{G}(U) / U$ and so $E_{0} \unlhd N_{G}(U)$. This proves the claim. So if $F$ is any hyperplane of $E_{0}$, letting $z^{g} \in F$, then we may take $g \in N\left(E_{0}\right)$. This gives $F \leqslant E_{0} \leqslant L_{0}^{g}$ so by the above $C_{G_{0}}(F) \leqslant N\left(E_{0}\right)$. Therefore (ii) holds and the case of $F_{4}(q)$ is complete.

Finally, consider ${ }^{2} E_{6}(4)$. By previous arguments we may take $E_{0}<L<\hat{L}$ and assume that each element of $E_{0}^{\#}$ is conjugate to $z$ and that $E_{0}$ normalizes no proper $r$-subgroup. Say $z \in E_{0}$ and set $D_{0}=C_{G_{0}}^{0}\left(E_{0}\right)$. By $\S 4 \hat{L}_{2} \leqslant D_{0}$, so $L_{2} \leqslant D_{0} \cap F_{4}(q)$. This holds for each element of $E_{0}^{\#}$. Now $L_{2}$ is generated by short root subgroups of $F_{4}(q)$, but as $q=4$ we can apply (2.8) to get the structure of $\left\langle L_{2}^{D_{0} \cap F_{4}(q)}\right\rangle=X$. Since $z \in L_{2} \leqslant X$, we have $E_{0} \leqslant X$. Analysis of the possible choices for $X$ and using $\boldsymbol{H}\left(r, E_{0}\right)=\{1\}$ leads to $X=F_{4}(q)$. But then $D_{0} \geqslant\left\langle F_{4}(q), \hat{L}_{2}\right\rangle=G_{0}$, so (i) holds. This completes the proof of (5.9).

(5.10) Let $G=E_{6}(q)$, or ${ }^{2} E_{6}(q)$ and $p=3 \mid q+1$. Then one of the following holds:

(i) $D_{0}=G_{0}$.

(ii) $p=3, G=E_{6}(2), E_{6}(4)$, or ${ }^{2} E_{6}(2)$, and there exists $E_{0}$ such that $C_{G_{0}}^{0}\left(E_{0}\right) \leqslant N_{G_{0}}\left(E_{0}\right)$.

Proof. By Table (5.2) together with (5.3)-(5.8) either $G={ }^{2} E_{6}(q)$ and $p=3 \mid q+1$ or $G=E_{6}(q)$ and $p=3$ or 5 with $p \mid q \pm 1$. First assume that $p \mid q+1$ and $G=E_{6}(q)$. Then $G_{0} \geqslant\left\langle U_{ \pm s}\right\rangle \times I_{0}$, where $s$ is the positive root of highest height and $I_{0} \cong S L(6, q)$. So $G_{0} \geqslant X$, where $X \cong S L(2, q) \times\left(S L(2, q)\right.$ wr $\left.Z_{3}\right)$. If $p=5$, then $X$ contains a Sylow 5-subgroup of $G_{0}$ so we may take $E_{0} \leqslant X$ and argue as in (5.5) to get $D_{0}=G_{0}$. Suppose $p=3$. By Table (5.2) we may assume $E_{0}<F_{4}(q)<E_{6}(q)$ and $E_{0}<L$, where $L$ is the second group listed in Table (3.3) for the group $E_{6}(q)$. In fact, from the proof of (3.3) we may assume that $F_{4}(q) \cap L$ is the second group listed for $F_{4}(q)$. If $q>2$, use (5.9), (4.1), and (4.5) to get $D_{0} \geqslant\left\langle L, F_{4}(q)\right\rangle$ and then apply (2.8) to obtain $\left\langle L, F_{4}(q)\right\rangle=G_{0}$. Suppose $q=2$. Here we choose $E_{0}<L_{0} \cap F_{4}(2)<L_{0} \cap F_{4}(4)$ as it was constructed in the $F_{4}(4)$ case of (5.9)(ii). The same arguments show that $D_{0} \leqslant N_{G_{0}}\left(E_{0}\right)$; hence (ii) holds.

If $p=5 \mid q-1$ we again may take $E_{0} \leqslant\left\langle U_{ \pm s}\right\rangle \times I_{0}$. Then $E_{0}$ projects to an abelian subgroup of $I_{0} \cong S L(6, q)$. Consequently $E_{0}$ acts reducibly on the usual module for $S L(6, q)$ and $И\left(r, E_{0}\right) \neq\{1\}$. So here (2.3) implies that (i) holds. At this point we may take $p=3$. If $G=E_{6}(q)$, then $3 \mid q-1$ and if $G={ }^{2} E_{6}(q)$, then $3 \mid q+1$. 
By (3.3) $G_{0}$ contains a subgroup $L$ such that $L$ contains a normal subgroup, $L_{0}$, of index 3 and $L_{0}=L_{1} L_{2} L_{3}$, a central product of three copies of $S L(3, q)$ or $S U(3, q)$ depending on whether $G_{0} \cong E_{6}(q)$ or ${ }^{2} E_{6}(q)$. Also $Z\left(L_{0}\right)=Z(L) \cong Z_{3} \times Z_{3}$. We write $\left\langle x_{i}\right\rangle=Z\left(L_{i}\right)$. Then $x_{i} \neq 1$ for $i=1,2,3$ and we may choose notation so that $x_{1}=x_{2} x_{3}^{-1}$ and $z=x_{1} x_{2}$ generates $Z\left(G_{0}\right)$. By Table (5.2) a Sylow 3-subgroup of $L$ has index 9 in a Sylow 3-subgroup of $G_{00}$. Here $G_{00}$ denotes the preimage in $\hat{G}$ of $\bar{G}_{\sigma}$. We claim that $N_{G_{00}}(L)$ contains a Sylow 3-subgroup of $G_{00}$. To see this we go back to the proof of Table (3.3), where $L$ was constructed. We had $X_{1}, X_{2}, X_{3}$ commuting copies of $S L(3, K)$ with centers $\left\langle z_{1}\right\rangle,\left\langle z_{2}\right\rangle,\left\langle z_{3}\right\rangle$, respectively. For each $i$ we constructed $a_{i} \in X_{i}$ with $a_{i}^{\sigma}=a_{i} z_{i}$. Then $a_{1} a_{2}^{-1} \in G_{00}-G_{0}$ and $a_{1} a_{2}^{-1} \in N(L)$. Also there was an element $g \in G_{0}$ interchanging $X_{1}$ and $X_{2}$, while normalizing $X_{3}$. Similarly, we can construct $g^{\prime} \in G_{0}$ interchanging $X_{2}$ and $X_{3}$, normalizing $X_{1}$. So $G_{0}$ contains a 3-element $x$ transitive on $\left\{X_{1}, X_{2}, X_{3}\right\}$. Then $L\left\langle x, a_{1} a_{2}^{-1}\right\rangle=\hat{L}$ contains a Sylow 3-subgroup of $G_{00}$ and $L<\hat{L}$. We may assume $E_{0}<\hat{L}$.

It is possible that $E_{0} \$ C\left(x_{1}\right)$, but we may assume $z \in E_{1}$ by (2.5), so $\left[x_{1}, E_{0}\right] \leqslant\langle z\rangle \leqslant E_{0}$. A slight extension of (2.9) allows us to take $x_{1} \in E_{0}$. We may assume that there exists $y \in E_{0}$ with $\left[x_{1}, y\right]=1$ and $y \notin\left\langle x_{1}, z\right\rangle$. To see this argue as follows. If $\left.E_{0} \cap C_{L}\left(x_{1}\right)\right\rangle\left\langle x_{1}, z\right\rangle$, just choose $y$ in the intersection. Otherwise, $E_{0}=\left\langle x_{1}, z, y\right\rangle$ for any $y \in E_{0}-\left\langle x_{1}, z\right\rangle$. But then $y$ is transitive on $\left\{X_{1}, X_{2}, X_{3}\right\}$, $\boldsymbol{\Lambda}\left(r, E_{0}\right) \neq\{1\}$, and by (2.3), (i) holds.

Suppose $q>4$ if $G=E_{6}(q)$ and $q>2$ if $G={ }^{2} E_{6}(q)$. Then $L_{0} \leqslant D_{0}$. Let $V$ be a long root subgroup of $G_{0}$ and set $I=\left\langle V^{G} \cap D_{0}\right\rangle$. By (3.4) $L_{0} \leqslant I$ and by (2.8) $I$ is a central product of finite groups of Lie type each defined over $\mathbf{F}_{q}$. Using order arguments we conclude that $I=L_{0}$. Also, $L_{0}=O^{r^{\prime}}\left(C_{G_{0}}\left(x_{1}\right)\right)$.

Since $C_{\hat{G}}\left(x_{1}\right)$ is connected we can embed $\left\langle x_{1}\right\rangle \times\langle y\rangle$ in a maximal torus $\hat{T}_{1}$ of $\hat{G}$. Let $\hat{T}_{1} \leqslant \hat{B}_{1}$, where $\hat{B}_{1}$ is a Borel subgroup of $\hat{G}$ and let $\hat{U}_{1}$ be the unipotent radical of $\hat{B}_{1}$. Then $\hat{U}_{1}$ is the product of 36 root subgroups and since $L_{0}=O^{r^{\prime}}\left(C_{G_{0}}\left(x_{1}\right)\right)$ we see that $x_{1}$ centralizes precisely 9 of these root subgroups. So some element of $\left\langle x_{1}, y\right\rangle-\left\langle x_{1}\right\rangle$ must centralize at least 9 root subgroups of $\hat{U}_{1}$ and we may take this element to be $y$. Using this information together with basic properties of the root system of type $E_{6}$ we have the following possibilities for the Dynkin diagram of $E\left(C_{\hat{G}}(y)\right): A_{2} \cup A_{2} \cup A_{2}, A_{1} \cup A_{4}, A_{1} \cup A_{5}, A_{5}, D_{4}, D_{5}$. As in the proof of (2.6) we see that the components of $C_{G_{0}}(y)$ are central extensions of some of the following groups: $L_{i}(q), 2 \leqslant i \leqslant 5, U_{i}(q), 2 \leqslant i \leqslant 5, L_{3}\left(q^{3}\right), U_{3}\left(q^{3}\right), U_{5}(q), D_{5}(q),{ }^{2} D_{5}(q)$, ${ }^{2} D_{4}(q), D_{4}(q),{ }^{3} D_{4}(q)$. Moreover, $q^{9}$ divides the order of $E\left(C_{G_{0}}(y)\right)$.

Let $J$ be a component of $C_{G_{0}}(y)$ and let $F=N_{E_{0}}(J)$. Suppose we knew that for any such $J, J=C_{J}^{0}(F)$. Then (2.16) implies that $E\left(C_{G_{0}}(y)\right) \leqslant D_{0}$. However, this contradicts the fact that $L_{0}=I=E\left(D_{0}\right)$. Therefore, there is some component $J$ with $J \neq C_{J}^{0}(F)$. By (4.1), (4.5) and induction we conclude that $F / C_{F}(J)$ is not contained in the subgroup of $\operatorname{Aut}(J)$ generated by inner and diagonal automorphisms. To handle the remaining cases we appeal to (6.1), (6.3), (6.4) and (6.5) of the next section. The proof of (6.1) does not require Theorem 1 . The proofs of (6.3), (6.4) and (6.5) make use of Theorem 1, but since we are in a minimal situation these applications are valid. Therefore, we conclude that $J=C_{J}^{0}(F)$, which is a contradiction. 
Suppose $G={ }^{2} E_{6}(2)$. Then $G_{0}$ has Sylow 3-subgroup of order $3^{10}$. Let $s$ be the positive root of highest height. Then $I=E\left(C_{G_{0}}\left(\left\langle U_{ \pm s}\right\rangle\right)\right) \cong S U(6,2)$. Now $I$ contains an elementary abelian subgroup, $F_{0}$, of order $3^{5}$ which is normal of index 9 in a Sylow 3-subgroup of $I$. Set $\langle v\rangle=O_{3}\left(\left\langle U_{ \pm s}\right\rangle\right)$ and $E_{0}=\langle v\rangle \times F_{0}$. We claim that $C_{G_{0}}^{0}\left(E_{0}\right) \leqslant N_{G_{0}}\left(E_{0}\right)$. Let $E_{1}$ be a hyperplane in $E_{0}$ with $v^{g} \in E_{1}$ for some $g \in G_{0}$. Then $E_{0} \leqslant C_{G_{0}}\left(E_{1}\right) \leqslant C_{G_{0}}(v) \cong Z_{3} \times S U(6,2)$. So $E_{0}$ projects to $E\left(C_{G_{0}}(v)\right)$ as an elementary abelian subgroup of order $3^{5}$. No proper parabolic subgroup of $S U(6,2)$ contains such a subgroup. By (4.1) and the proof of (4.2), $C_{G_{0}}\left(E_{1}\right) \leqslant N\left(E_{0}\right)$.

Now suppose $E_{1}$ is a hyperplane of $E_{0}$ containing no $G_{0}$-conjugate of $v$. Then $E_{0}=\langle v\rangle \times E_{1}$ and $E_{1}$ projects to $F_{0}$ in $I$. Let $M$ be the natural module for $S U(6,2)$ and let $\left\{v_{1}, \ldots, v_{6}\right\}$ be an orthonormal basis for $M$. We may asume $F_{0}$ is diagonal in the basis and $F_{0}=\left\langle x_{1}\right\rangle \times \cdots \times\left\langle x_{5}\right\rangle$, where $\left(v_{i}\right) x_{i}=\alpha v_{i},\left(v_{i+1}\right) x_{i}=\alpha^{-1} v_{i+1}$, and $\left(v_{j}\right) x_{i}=v_{j}$ if $j \neq i, i+1$. Here $\alpha \in \mathbf{F}_{4}$ is an element of order 3. Each $x_{i}$ is $G_{0}$-conjugate to $v$, so for each $i$ either $v x_{i}$ or $v^{-1} x_{i}$ is an element of $E_{1}$. Say $v x_{1} \in E_{1}$. If $v^{-1} x_{2} \in E_{1}$, then $x_{1} x_{2} \in E_{1}$. However, we have $x_{1} x_{2} \sim x_{1}$ in $I$, a contradiction. So $v x_{2} \in E_{1}$. Similarly, $v x_{3} \in E_{1}$. Then $\left(v x_{1}\right)\left(v x_{2}\right)\left(v x_{3}\right)=x_{1} x_{2} x_{3} \in E_{1}$ and again we have $E_{1} \cap v^{G_{0}} \neq \varnothing$. This is a contradiction. So $D_{0} \leqslant N\left(E_{0}\right)$.

Finally, we consider $G=E_{6}(4)$. Let $\tilde{L}=L\langle x\rangle$. We claim that there exists $e \in \tilde{L}-L$ with $|e|=3$. To see this consider the natural embedding of $G_{0}$ in $E_{7}(4)$. The group $N$ of the Tits' system for $E_{7}(4)$ splits over the split torus, $T$. To see this just observe that the long word in the fundamental generators of the Weyl group of $E_{7}(4)$ inverts $T$. The splitting of $N$ over $T$ forces a similar splitting in $G_{0}$, and this guarantees the existence of $e$.

We may assume $X_{1}^{e}=X_{2}$ and $X_{2}^{e}=X_{3}$. So $E\left(C_{L_{0}}(e)\right) \cong P S L(3,4)$. Let $\langle b, c\rangle \in \operatorname{Syl}_{3}\left(E\left(C_{L_{0}}(e)\right)\right)$ and set $E_{0}=\left\langle x_{1}, x_{2}, b, c\right\rangle$. We fix a hyperplane, $F$, of $E_{0}$ and claim that $C_{G_{0}}(F) \leqslant N_{G}\left(E_{0}\right)$. Clearly, we may assume $z \in F$.

Suppose, $x_{1} \in F$. As $N_{G}\left(E_{0}\right)$ is transitive on $\left(E_{0} /\left\langle x_{1}, x_{2}\right\rangle\right)^{\#}$, we may assume $F=\left\langle z, x_{1}, b\right\rangle=\left\langle x_{1}, x_{2}, b\right\rangle$. Then

$$
C_{G_{0}}(F) \leqslant C_{G_{0}}\left(x_{1}\right)=L .
$$

Write $b=b_{1} b_{2} b_{3}$ and $c=c_{1} c_{2} c_{3}$, where $b_{i}, c_{i} \in L_{i}$ for $i=1,2,3$, and let $V=$ $\left\langle x_{1}, x_{2}, b_{1}, b_{2}, b_{3}, c\right\rangle=C_{L_{0}}(b)$. Then $C_{G_{0}}(F)=C_{L}(b)=V\langle d\rangle$ for $d \in L-L_{0}$. Now $e$ normalizes $V\langle d\rangle$ and we consider $V\langle e, d\rangle /\left\langle x_{1}, x_{2}\right\rangle$. The group $V /\left\langle x_{1}, x_{2}\right\rangle$ is elementary abelian and $d$ normalizes $V /\left\langle x_{1}, x_{2}\right\rangle \cap C(e)=\left\langle b, c, x_{1}, x_{2}\right\rangle /\left\langle x_{1}, x_{2}\right\rangle$. On the other hand, $\left\langle\left[d, c_{i}\right]\right\rangle\left\langle x_{i}\right\rangle=\left\langle b_{i}, x_{i}\right\rangle$, for $i=1,2,3$ and this implies that $[d, c] \leqslant\left\langle b, x_{1}, x_{2}\right\rangle=F$. We conclude that $F=C_{G_{0}}(F)^{\prime}=C_{L}(b)^{\prime}$. In particular, $E_{0} \unlhd C_{L}(b)=C_{G_{0}}(F)$.

Viewing $C_{G_{0}}(b) \cap C_{G_{0}}\left(x_{1}\right)$ first as a subgroup of $L$ and then as a subgroup of $C_{G_{0}}(b)$, we conclude from [7] that $b \sim x_{1}$ in $G_{0}$. Let $U=\left\langle z, x_{1}, b\right\rangle$ and $D=N_{G_{0}}(U)$ $\cap C(U /\langle z\rangle)$. Then $D$ is a 3-group of index 3 in a Sylow 3-subgroup of $C_{G_{0}}\left(\left\langle x_{1}, z\right\rangle /\langle z\rangle\right)$ and in a Sylow 3-subgroup of $C_{G_{0}}(\langle b, z\rangle /\langle z\rangle)$. It follows that $O^{2^{\prime}}\left(N_{G_{0}}(U)\right)$ induces $S L(2,3)$ on $U /\langle z\rangle$.

We have $C_{G_{0}}(U)=\left\langle U, b_{1}, b_{2}, c, d\right\rangle$. Also,

$$
\left\langle c_{1}, c_{2}, c_{3}, z\right\rangle\langle e\rangle=\left\langle c_{1}, c_{1}^{e}, c_{1}^{e^{2}}, z\right\rangle\langle e\rangle,\left\langle b_{1}, b_{2}, b_{3}, z\right\rangle\langle e\rangle=\left\langle b_{1}, b_{1}^{e}, b_{1}^{e^{2}}, z\right\rangle\langle e\rangle,
$$


and both groups are isomorphic to $Z_{2} \times\left(Z_{3} \mathrm{wr} Z_{3}\right)$. Set $b_{0}=\left[b_{1}, e\right]$ and $c_{0}=\left[c_{1}, e\right]$. Then $D=C_{G_{0}}(U)\left\langle e, c_{0}\right\rangle$. We will use the fact that $\left\langle z, b, b_{0}\right\rangle$ is the unique hyperplane of $\left\langle z, b_{1}, b_{2}, b_{3}\right\rangle$ that contains $z$ and is normalized by $e$.

Now $D / C_{G_{0}}(U)$ acts on $U \cong Z_{3} \times Z_{3} \times Z_{3}$ as the group of transvections in $S L(3,3)$ with fixed direction (namely $\langle z\rangle$ ). Hence, $O^{2^{\prime}}\left(N_{G_{0}}(U)\right) / C_{G_{0}}(U)$ is isomorphic to $O^{2^{\prime}}(P)$, for $P$ a parabolic subgroup of $S L(3,3)$. In particular, $O^{2^{\prime}}\left(N_{G_{0}}(U)\right)$ is transitive on $\left(D / C_{G_{0}}(U)\right)^{\#}$. Consider the action of $D$ on $C_{G_{0}}(U) / U$ $=\left\langle\bar{b}_{1}\right\rangle \times\left\langle\bar{b}_{2}\right\rangle \times\langle\bar{c}\rangle \times\langle\bar{d}\rangle$, where bars denote images modulo $U$. Then $c_{0}$ induces a transvection on this elementary group having centralizer $\left\langle\bar{b}_{1}, \overline{b_{2}}, \bar{c}\right\rangle$ and commutator space in $\left\langle\bar{b}_{1}, \bar{b}_{2}\right\rangle$. This commutator space is normalized by $e$, so earlier remarks imply that $\left[C_{G_{0}}(U) / U,\left\langle c_{0}\right\rangle\right]=\left\langle\bar{b}_{0}\right\rangle$. We must have $e$ inducing a transvection on $C_{G_{0}}(U) / U$, and since $\left[b_{1}, e\right]=b_{0}$, we conclude that $\left[C_{G_{0}}(U) / U,\langle e\rangle\right]=\left\langle\bar{b}_{0}\right\rangle$, as well. It follows that $\left[D, C_{G_{0}}(U)\right] U / U=\left\langle\bar{b}_{0}\right\rangle \unlhd O^{2}\left(N_{G_{0}}(U)\right)$. Moreover, $O^{2^{\prime}}\left(N_{G}(U)\right)$ normalizes $Z(D / U) \cap C_{G_{0}}(U) / U=\left\langle\overline{b_{0}}, \bar{c}\right\rangle$. Therefore, $O^{2^{\prime}}\left(N_{G_{0}}(U)\right) \leqslant$ $C\left(\left\langle b_{0}, c, U\right\rangle / U\right)$, so $O^{2^{\prime}}\left(N_{G_{0}}(U)\right) \leqslant N\left(E_{0}\right)$. Consequently, there is an element $g \in$ $N\left(E_{0}\right)$ such that $x_{1} \in F^{g}$. By the previous case, $C_{G_{0}}\left(F^{g}\right)>N\left(E_{0}\right)$; whence $C_{G_{0}}(F) \leqslant$ $N\left(E_{0}\right)$. This proves the claim. Therefore, (ii) holds and the proof of $(5.10)$ is complete.

(5.11) Suppose $G=E_{7}(q)$ or $E_{8}(q)$. Then one of the following holds:

(i) $D_{0}=G_{0}$.

(ii) $q=2$ and $p=3$.

(iii) $q=4, p=3$, and $G_{0}=E_{8}(4)$.

Moreover, if $p=3$ and $G_{0}=E_{7}(2), E_{8}(2)$, or $E_{8}(4)$, then there is an elementary abelian 3-subgroup $E_{0} \leqslant G_{0}$ such that $C_{G_{0}}^{0}\left(E_{0}\right) \leqslant N_{G_{0}}\left(E_{0}\right)$.

Proof. Since $p \nmid\left|Z\left(G_{0}\right)\right|, E_{0} \cong E$ is elementary abelian. By Table (5.2) and (5.3)-(5.8) we need only consider the cases $p=3,5,7$ and $p \mid q \pm 1$. Regard $I=E_{7}(q) \leqslant E_{8}(q)=G_{0}$ in the obvious way. Let $J=\left\langle U_{ \pm s}\right\rangle$ for $s$ the positive root of highest height in $G_{0}=E_{8}(q)$. Then $I=E\left(C_{G_{0}}(J)\right)$.

Suppose $p=7$. Then Table (5.2) implies that $J I$ contains a Sylow $p$-subgroup of $G_{0}$. If $p \mid q-1$ then a Sylow $p$-subgroup of $I$ is contained in a parabolic subgroup of $I$ with a section of type $L_{7}(q)$. So here (2.3) gives (i). Suppose $p \mid q+1$. By Table (3.3) and (3.4) $E_{8}(q)$ contains a central extension of $U_{9}(q)=X$ with $X$ generated by root subgroups of $E_{8}(q)$. Then $X$ contains a conjugate, $J^{g}$, of $J$ such that $C_{X}\left(J^{g}\right) \geqslant O^{7^{\prime}}(G U(7, q))$. So $I \geqslant O^{7^{\prime}}(G U(7, q))$ and has Sylow 7-subgroups isomorphic to $O_{7}\left(Z_{q+1}\right)$ wr $Z_{7}$. Let $S \in \operatorname{Syl}_{7}(I)$ or $S \in \operatorname{Syl}_{7}(J I)$, according to $G_{0}=E_{7}(q)$ or $E_{8}(q)$, and let $S_{0}$ be the unique subgroup of $S$ with $S_{0}$ the direct product of 7 (respectively, 8) copies of $O_{7}\left(Z_{q+1}\right)$. By (2.3) and the argument used to prove (4.2) we may assume that $E_{0} \leqslant S_{0}$. On the other hand, $I$ contains the direct product, $J_{1} \times \cdots \times J_{7}$, of seven conjugates of $J$. Therefore, we may assume $E_{0} \leqslant J_{1} \cdots J_{7}$ (respectively, $J J_{1} \cdots J_{7}$ ) and argue as in (5.5) to get (i).

Suppose $G_{0}=E_{7}(q)$ and $p \mid q-1$. Here $p=3$ or 5 and a Sylow $p$-subgroup of $G_{0}$ is contained in a conjugate of the parabolic subgroup of $G_{0}$ having an $E_{6}(q)$ section. So $И\left(r, E_{0}\right) \neq\{1\}$ and (i) holds by (2.3).

Next suppose $G_{0}=E_{7}(q)$ and $p \mid q+1$, where $p=3$ or 5 . If $p=5$, then let $J_{1}$ and $L \cong O^{5^{\prime}}(G U(7, q))$ be as in the case $p=7$. By orders we may assume that 
$E_{0} \leqslant J_{1} L$, and arguing as before we have $E_{0}$ conjugate to a subgroup of $J_{1} \cdots J_{7}$, where the product is a commuting product of conjugates of $J_{1}$. Once again argue as in (5.5) to get (i). Suppose $p=3$. By Table (3.3) $G_{0}$ contains a subgroup $R$ such that $E(R)$ is the covering group of ${ }^{2} E_{6}(q)$ and $R / E(R) \cong Z(R) \cong Z_{3}$. Then $R$ contains a Sylow 3-subgroup of $G_{0}$, so we may take $E_{0}<R$. Also $E_{0} \cong E$ is elementary abelian. Recall the subgroup $L$ discussed in (5.10) and regard $E_{0} \leqslant N_{G_{0}}(L)$. By (2.9) we may take $z \in E_{0}$, where $\langle z\rangle=Z(L)$. Assume $q>2$. Let $e \in E_{0}-\langle z\rangle$ and set $E_{1}=\langle e, z\rangle$. By (2.16), (2.6)(ii), (2.5), (4.1) and results of this section, $O^{r^{\prime}}\left(C_{G_{0}}(t)\right) \leqslant D_{0}$ for each $t \in E_{1}^{\#}$. By (5.10) and (3.4) $E(R) \leqslant I$, where $I=\left\langle V^{G_{0}} \cap D_{0}\right\rangle$ and $V$ is a root subgroup of $G_{0}$. Then, assuming (i) fails, (2.8) and a check yield $E(R)=I=O^{r^{\prime}}\left(E\left(D_{0}\right)\right)$. We then must have $O^{r^{\prime}}\left(C_{G_{0}}(t)\right) \leqslant E(R)$ for each $t \in E_{1}^{\#}$. Now argue (see (2.7)(ii) and (2.11) for example) that $t \in E_{1}-\langle z\rangle$ may be chosen with $O^{r^{\prime}}\left(C_{R}(t)\right) \neq 1$. Then $И\left(r, E_{1}\right) \neq\{1\}, C_{G_{0}}^{r}\left(E_{1}\right)=G_{0}$ by (2.3), and we have a contradiction. This completes $G_{0}=E_{7}(q)$ except for $q=2, p=3$.

Suppose $G_{0}=E_{8}(q)$ and $p=5$. By Table (3.3) $G_{0}$ contains a subgroup $Y$ such that $E(Y)$ is the central product of two copies of $S L(5, q)$ or $S U(5, q)$, according to $5 \mid q-1$ or $5 \mid q+1$. Also $Y / E(Y) \cong Z_{5}$ and, by Table (5.2), $Y$ contains a Sylow 5-subgroup of $G_{0}$. We take $E_{0}<Y$, and let $J$ and $I$ be as before. Since a Sylow 5 -subgroup of $I J$ has index 5 in one for $G_{0}$, we may assume $I J$ contains a hyperplane, say $F$, of $E_{0}$. Suppose $F$ contains $F_{0} \cong Z_{5} \times Z_{5}$. As in (5.10) there are commuting $G_{0}$-conjugates $J_{1}, \ldots, J_{7}$ of $J$ such that $F_{0} \leqslant J J_{1} \cdots J_{7}=J_{0}$. Clearly $J_{0}=C_{J_{0}}^{r}\left(F_{0}\right)$, so by $(2.16),(4.1)$, and the results of this section, we have $J_{0} \leqslant D_{0}$. But, also, (4.1) implies $E(Y) \leqslant D_{0}$. We can now use (2.8) to argue that $D_{0}=G_{0}$. So suppose $F \cong Z_{5}$, that is $E_{0} \cong Z_{5} \times Z_{5}$. By (2.9) we may assume $z \in E_{0}$, where $\langle z\rangle=Z(Y)$. From the structure of $S L(5, q)$ and $S U(5, q)$ it is clear that $E_{0}$ acts on an elementary abelian subgroup of $E(Y)$ of order $5^{7}$. It follows that $E_{0}$ can be embedded in an elementary subgroup of order $5^{3}$. So using (2.9) we reduce to the previous case, and obtain $D_{0}=G_{0}$. From now on we have $p=3$.

Let $G_{0} \cong E_{8}(q)$ and use Table (3.3) to get a subgroup $L_{1} \leqslant G_{0}$ such that $E\left(L_{1}\right)=A_{0} B_{1}$, a central product, where $A_{0} \cong S L(3, q)$ or $S U(3, q), B_{1} \cong \hat{E}_{6}(q)$ or ${ }^{2} \hat{E}_{6}(q)$ and in each case the choice is determined by whether $q \equiv 1$ or $-1(\bmod 3)$. Also $Z\left(L_{1}\right)=\langle z\rangle \cong Z_{3}$ and $L_{1} / E\left(L_{1}\right) \cong Z_{3}$. By Table (5.2) $L_{1}$ contains a Sylow 3-subgroup of $G_{0}$, so we may take $E_{0} \leqslant L_{1}$. There is an element $x_{1} \in B_{1}$ such that $\left|x_{1}\right|=3$ and $E\left(C_{B_{1}}(x)\right)=A_{1} A_{2} A_{3}$, a central product of three $G_{0}$-conjugates of $A_{0}$ (see Table (3.3)). We set $A=A_{0} A_{1} A_{2} A_{3}$ and choose notation so that $x_{1} \in A_{1}$. Then $Z(A)=\langle z\rangle \times\left\langle x_{1}\right\rangle$.

We also have $N_{L_{1}}(A)$ containing a subgroup $\hat{A}>A$ with $\hat{A} / A \cong Z_{3} \times Z_{3}$ and $\hat{A}$ normalizing each $A_{i}$. Finally, there is a 3-element $e_{1} \in N_{L_{1}}(A)$ such that $\hat{A}=\hat{A}^{e_{1}}$ and $e_{1}$ permutes $\left\{A_{1}, A_{2}, A_{3}\right\}$ as the 3 -cycle $(1,2,3)$. By orders $\hat{A}\left\langle e_{1}\right\rangle$ contains a Sylow 3-subgroup of $G_{0}$, so we choose $E_{0} \leqslant \hat{A}\left\langle e_{1}\right\rangle$.

First suppose $q>4$. If $E_{0} \leqslant \hat{A}$, then let $E_{1}=\left\langle z, x_{1}\right\rangle$. If $E_{0} \neq \hat{A}$, let $E_{1}=\langle z, e\rangle$, where $e$ is any element in $E_{0}-\hat{A}$. Then $E_{0} \leqslant C\left(E_{1}\right)$. In either case it is clear that $E_{1}$ centralizes a proper $r$-subgroup of $G_{0}$, so (2.3) gives $C_{G_{0}}^{r}\left(E_{1}\right)=G_{0}$. But the assumption $q>4$ together with (4.1), (4.5), and the results of this section give $C_{G_{0}}^{r}\left(E_{1}\right) \leqslant D_{0}$. So (i) holds in this case. 
Suppose, now, that $q=4, p=3$, and $G_{0}=E_{8}(4)$. We will produce a subgroup $E_{0}$ such that (iii) holds. Retain the above notation. Thus $E\left(L_{1}\right) \cong S L(3,4) \cdot \hat{E}_{6}(4)$. Set $x_{2}=x_{1}^{e_{1}}$ and $x_{3}=x_{2}^{e_{1}}$. Then $x_{1} x_{2} x_{3}=1$, so $x_{3}=x_{1}^{-1} x_{2}^{-1}$ and we may assume that $z=x_{1} x_{2}^{-1}$. Set $S \in \operatorname{Syl}_{3}\left(\hat{A}\left\langle e_{1}\right\rangle\right)$ with $e_{1} \in S$, and write $S_{i}=S \cap A_{i}$ for $i=0,1,2,3$. Then $S_{i}=\left\langle a_{i}, b_{i}\right\rangle$, where $\left[a_{i}, b_{i}\right]=x_{i}$ for $i=1,2,3,\left[a_{0}, b_{0}\right]=z$, and $e_{1}$ stabilizes $\left\{a_{1}, a_{2}, a_{3}\right\}$ and $\left\{b_{1}, b_{2}, b_{3}\right\}$. We now set $E_{0}=\left\langle z, x_{1}, a_{0} a_{1} a_{2} a_{3}, b_{1} b_{2} b_{3}, b_{0} b_{1}^{-1} b_{2}\right\rangle$ and observe that $E_{0}$ is elementary of order $3^{5}$. We will show that $C_{G_{0}}^{0}\left(E_{0}\right) \leqslant N_{G}\left(E_{0}\right)$.

Let $F$ be a hyperplane in $E_{0}$ and assume $z \in F$. First, suppose $x_{1} \in F$. Then $C_{G_{0}}(F) \leqslant C_{G_{0}}\left(x_{1}\right) \cap C_{G_{0}}(z)=C_{L_{1}}\left(x_{1}\right)=\hat{A}$. Calculation within $\hat{A}$ yields $C_{G_{0}}(F) \leqslant$ $N_{G_{0}}\left(E_{0}\right)$. Suppose $F$ does not contain $x_{1}$, so that $E_{0}=F \times\left\langle x_{1}\right\rangle$. Using the results of [7] one checks that $\left\langle b_{1} b_{2} b_{3}, x_{1}\right\rangle^{\#}$ is fused in $B_{1} \cong \hat{E}_{6}(4)$. Let $\langle x\rangle=F \cap\left\langle b_{1} b_{2} b_{3}, x_{1}\right\rangle$. Then $E_{0} \leqslant C_{L_{1}}(x) \sim C_{L_{1}}\left(x_{1}\right)$ and

$$
C_{G_{0}}(F) \leqslant C_{G_{0}}(\langle z, x\rangle)=\hat{A}^{g}
$$

for some $g \in B_{1}$. Since $x_{1} \notin F, F$ projects onto a Sylow 3-subgroup of $A_{0} / Z\left(A_{0}\right)$, and it follows that $C_{G_{0}}(F) \leqslant\left(A\left\langle g_{1}\right\rangle\right)^{g}$, where $g_{1} \in \hat{A} \cap B_{1}$.

Let $Q=\left\langle z, x_{1}, a_{1} a_{2} a_{3}, b_{1} b_{2} b_{3}\right\rangle$. As in the $E_{6}(4)$ case, we have $(Q /\langle z\rangle)^{\#}$ fused in $N_{B_{1}}(Q)$. So we may take $g \in N_{B_{1}}(Q)$ such that $x_{1}^{g}=x$ and $Q \leqslant\left(A_{1} A_{2} A_{3}\right)^{g}$. We claim that $C_{G_{0}}(F)$ is contained in a Sylow 3-subgoup, $Y$, of $\left(A\left\langle g_{1}\right\rangle\right)^{g}$. It will suffice to show that $C_{\left(A_{1} A_{2} A_{3}\right)^{g}}(\tilde{F})$ is a 3-group, where $\tilde{F}$ is the projection of $F \cap A^{g}$ to $\left(A_{1} A_{2} A_{3}\right)^{g}$. However, $C_{A_{1} A_{2} A_{3}}\left(\left\langle x_{1}, z, q_{1}\right\rangle\right)$ is a 3 -group for any $q_{1} \in Q-\left\langle x_{1}, z\right\rangle$ and $\left.\tilde{F} \cap Q^{g}\right\rangle\left\langle x_{1}^{g}, z\right\rangle$. This proves the claim.

Suppose $Y=Y_{0}\langle e\rangle$ for some $e \in F$, where $Y_{0}=Y \cap A^{g}$. If $y \in C_{Y}(F)$, then $y=e^{i} y_{0}$ for some $y_{0} \in Y_{0}$. So

$$
E_{0}^{y}=E_{0}^{y_{0}}=\left(\langle e\rangle\left(E_{0} \cap Y_{0}\right)\right)^{y_{0}}=\langle e\rangle^{y_{0}}\left(E_{0} \cap Y_{0}\right)^{y_{0}}=\langle e\rangle\left(E_{0} \cap Y_{0}\right)=E_{0}
$$

and $y \in N_{G_{0}}\left(E_{0}\right)$. Suppose no such element, $e$, exists. Then $F \leqslant Y_{0}$. The projection of $E_{0}$ to $B_{1}$ is nonabelian, so the projection of $F$ to $B_{1}$ is nonabelian. This forces $C_{Y}(F) \leqslant Y_{0}$. In particular, $E_{0} \leqslant Y_{0}$ and so $C_{Y}(F) \leqslant N\left(E_{0}\right)$. We have now shown that $C_{G_{0}}(F) \leqslant N_{G_{0}}\left(E_{0}\right)$, whenever $F$ is a hyperplane of $E_{0}$ containing $z$.

If $F$ is a hyperplane of $E_{0}$ with $E_{0}=F\langle z\rangle$, then $F$ contains $x_{1} z^{i}=x$, for some $i$. As $A \leqslant C(x)$ we use [7] to conclude that $x \sim z$. So $C_{G_{0}}(x) \sim C_{G_{0}}(z)$ and $A \leqslant C_{G}(x)$. In view of the embedding of $E_{0}$ in $A$ we can argue as above that $C(F) \leqslant N\left(E_{0}\right)$. This completes the proof that $C_{G_{0}}^{0}\left(E_{0}\right) \leqslant N_{G_{0}}\left(E_{0}\right)$ for this case.

We are left with the cases $G_{0}=E_{7}(2)$ or $E_{8}(2)$ and $p=3$. Let $s$ be the positive root of highest height and $J=\left\langle U_{ \pm s}\right\rangle \cong S_{3}$. Then $C_{G_{0}}\left(O_{3}(J)\right)=O_{3}(J) \times E\left(C_{G_{0}}(J)\right)$ and $E\left(C_{G_{0}}(J)\right) \cong O^{+}(12,2)^{\prime}$ or $E_{7}(2)$, according to whether $G_{0} \cong E_{7}(2)$ or $E_{8}(2)$. In the former case $E\left(C_{G_{0}}(J)\right)$ contains $J_{1} \times \cdots \times J_{6}$, a direct product of $6 G_{0}$-conjugates of $J$. To see this just consider the direct product of three copies of $O^{+}(4,2)$ in $O^{+}(12,2)$. If $G_{0}=E_{8}(2)$ let $J_{0}$ be a conjugate of $J$ in $E\left(C_{G_{0}}(J)\right)$. Then $G_{0}$ contains $J \times J_{1} \times \cdots \times J_{6}$ or $J \times J_{0} \times J_{1} \times \cdots \times J_{6}$, depending on whether $G_{0}=E_{7}(2)$ or $E_{8}(2)$. Let $O_{3}(J)=\langle x\rangle$ and $O_{3}\left(J_{i}\right)=\left\langle x_{i}\right\rangle$ for $i=1, \ldots, 6 \quad(i=0, \ldots, 6$ if $G_{0} \cong E_{8}(2)$ ). 
We claim that $C_{G_{0}}^{0}\left(E_{0}\right) \leqslant N_{G_{0}}\left(E_{0}\right)$, where $E_{0}=\left\langle x, x_{1}, \ldots, x_{6}\right\rangle$ or $\left\langle x, x_{0}, x_{1}, \ldots, x_{6}\right\rangle$, depending on whether $G_{0} \cong E_{7}(2)$ or $E_{8}(2)$. First suppose $G_{0} \cong E_{7}(2)$. Let $F$ be maximal in $E_{0}$ with $x \in F$. Then by (4.1) we have $C_{G_{0}}(F)=C_{G_{0}}(F) \cap C_{G_{0}}(x) \leqslant N_{G_{0}}\left(E_{0}\right)$. Now suppose $E_{0}=\langle x\rangle \times F$. As above we may assume $\left\langle x_{i}\right\rangle \neq F$ for $i=1,2$. So $x^{m} x_{1}, x^{n} x_{2} \in F$ for $m, n \in\{1,-1\}$ and $a \in F$ for $a=x_{1} x_{2}$ or $x_{1} x_{2}^{-1}$. Now $C(a) \cap E\left(C_{G_{0}}(J)\right)=\langle a\rangle \times O^{-}(10,2)^{\prime}$, so $C_{G}(a) \cap C(J)=\langle a\rangle \times O^{-}(10,2)^{\prime}$. From Burgoyne [7] we conclude that $C_{G_{0}}(a)=$ $\langle a\rangle \times J \times O^{-}(10,2)^{\prime}$. Again we use (4.1) to argue that $C_{G_{0}}(F) \leqslant N_{G_{0}}\left(E_{0}\right)$. So $C_{G_{0}}^{0}\left(E_{0}\right) \leqslant N_{G_{0}}\left(E_{0}\right)$.

Now suppose $G_{0} \cong E_{8}(2)$. Here we argue as above. First let $F$ be maximal in $E_{0}$ with $x \in F$. Then $C_{G_{0}}(F) \leqslant C_{G_{0}}(x)=\langle x\rangle \times E_{7}(2)$, so from the above paragraph we conclude that $C_{G_{0}}(F) \leqslant N_{G_{0}}\left(E_{0}\right)$. To complete the proof argue as above, using [7] to show that $E\left(C_{G_{0}}(a)\right) \cong O^{-}(14, q)$. This yields $C_{G_{0}}^{0}\left(E_{0}\right) \leqslant N_{G_{0}}\left(E_{0}\right)$ and completes the proof of (5.11).

(5.12) Let $G=G_{2}(q)$. Then one of the following holds:

(i) $D_{0}=G_{0}$.

(ii) $q=2, p=3, G_{0} \cong \operatorname{Aut}(\operatorname{PSU}(3,3))$ and $D_{0}$ normalizes a Sylow 3-subgroup of $G_{0}$, for any proper 3-subgroup, $E_{0}$, of $G_{0}$.

Proof. By (5.3), (5.8), and Table (5.2) we may assume $p=3$. From Table (3.3) we see that $G_{0}$ contains a subgroup $L$ with $L \cong S L(3, q)$ or $S U(3, q)$, according to whether $3 \mid q-1$ or $3 \mid q+1$. Then $L$ contains a Sylow 3 -subgroup of $G_{0}$, and we take $E_{0}<L$. If $3 \mid q-1$, then $L \cong S L(3, q)$ has just one class of elementary groups of order $3^{n}>3$. They are isomorphic to $Z_{3} \times Z_{3}$ and are conjugate to a subgroup of $H$. So (i) holds by (2.3).

Now suppose $3 \mid q+1$ and $q>2$. Again $E_{0} \cong Z_{3} \times Z_{3}$ and $Z(L)<E_{0}$. So $L \leqslant D_{0}$. For $e \in E_{0}-Z(L), e$ is contained in $\left\langle U_{ \pm s}\right\rangle^{g} \leqslant L$ for some $g \in G$, where $s$ is the positive (long) root of highest height (to see this check the action of $e$ on the usual module for $S U(3, q))$. Then $E_{0}$ acts on $E\left(C_{G_{0}}\left(\left\langle U_{ \pm s}\right\rangle^{g}\right)\right)=\left\langle U_{ \pm \alpha_{1}}\right\rangle^{g}$. Consequently $\left\langle U_{ \pm \alpha_{1}}^{g}\right\rangle \leqslant D_{0}$, and usual arguments (e.g. apply (2.8)) show that $G_{0}=\left\langle L,\left\langle U_{ \pm \alpha_{1}}\right\rangle^{g}\right\rangle$. Again (i) holds.

For $q=2$, just note that $G_{2}(q) \cong \operatorname{Aut}\left(U_{3}(3)\right)$, so (ii) holds. This proves (5.12).

(5.13) Let $G_{0}={ }^{3} D_{4}(q)$. Then $D_{0}=G_{0}$.

Proof. By (5.3), (5.7) and Table (5.2) we may assume that $p$ is associated with $\Phi_{3}$ or $\Phi_{6}$, or that $p=3$. Assume $q \neq 2$. By Table (3.3) $G_{0}$ contains a subgroup $L$ with the following properties: $O^{2}(L) \cong S L(3, q)$ if $3 \mid q-1$ or if $p$ is associated with $q^{3}-1 ; O^{2}(L) \cong S U(3, q)$ if $3 \mid q+1$ or if $p$ is associated with $q^{6}-1 ; L=O^{2^{\prime}}(L) C$ where $C \cong q^{2}+q+1$ or $q^{2}-q+1$ depending on whether $O^{2^{\prime}}(L) \cong S L(3, q)$ or $S U(3, q) ;[L, C]=1$ and $L \cap C=Z(L)$.

Suppose $p$ is associated with $q^{3}-1$ or $q^{6}-1$. Then $L$ contains a Sylow $p$-subgroup of $G_{0}$ and we take $E_{0} \leqslant L$. Then $E_{0} \cong Z_{p} \times Z_{p}$ and $E_{0}=\left(E_{0} \cap E(L)\right) \times\left(E_{0} \cap C\right)$. At this point we can argue as in (5.8) to conclude $D_{0}=G_{0}$.

Suppose, then, that $p=3$. Again using Table (3.3) we see that $L<\bar{L}$ with $\bar{L} / L \cong Z_{3}$. Then $\bar{L}$ contains a Sylow 3 -subgroup of $G_{0}$ and we take $E_{0}<\bar{L}$. Also $G_{0}$ 
contains $\left\langle U_{ \pm \alpha_{2}}\right\rangle \times\left\langle U_{ \pm s}\right\rangle$, for $s$ the positive root of highest height, and this group is isomorphic to $S L(2, q) \circ S L\left(2, q^{3}\right)$. Thus a Sylow 3-subgroup of $G_{0}$ contains a group isomorphic to $O_{3}\left(Z_{q \pm 1}\right) \times O_{3}\left(Z_{q^{3} \pm 1}\right)$. It follows from these facts that $G_{0}$ has 3-rank 2 and $E_{0}=\langle z, y\rangle$ for $\langle z\rangle=Z(L)$. In particular $\bar{L} \leqslant D_{0}$.

Let $I=\left\langle V^{G} \cap D_{0}\right\rangle$, where $V$ is a root subgroup for a long root. Then $O^{2^{\prime}}(L) \leqslant I<D_{0}$. Using (2.8) together with the fact that $I \leqslant D_{0} \leqslant N(I)$, we have $I=O^{2^{\prime}}(L)$. So $O^{2^{\prime}}(L) \unlhd D_{0}$ and hence $D_{0} \leqslant N(\langle z\rangle)$. Also (2.7) and (2.11) imply that we may choose $y$ such that $K=E\left(C_{\hat{G}}(y)\right) \neq 1$. Then $K_{\sigma} \leqslant D_{0} \leqslant N(\langle z\rangle)$. Consequently, (2.3) implies the result, unless $S_{3} \cong O^{2^{\prime}}\left(K_{\sigma}\right) \$ C(z)$. However, from Burgoyne [7] we see that $O^{2}\left(K_{\sigma}\right) \cong S_{3}$ is impossible.

The remaining case is $q=2$. We have $G_{0} \geqslant L \cong S U(3,2)$ and $G_{0} \geqslant X \cong L_{2}(2) \times L_{2}(8)$. Let $S_{0}=O_{3}(L)$. Then $S_{0}$ has index 3 in a Sylow 3-subgroup $S$ of $G_{0}$ and $S \leqslant N_{G_{0}}(L)$. For $s \in S-S_{0}, S=S_{0}\langle s\rangle$ and since $S$ has exponent 9 , $|s|=9$. Thus $E_{0}<S_{0}$ and $G_{0}$ contains one class of subgroups isomorphic to $Z_{3} \times Z_{3}$. We may take $E_{0} \leqslant X \cap L$ so that $\langle X, L\rangle \leqslant D_{0}$. Moreover, $E_{0} \leqslant L$ implies that $E_{0}-\langle z\rangle$ is fused, so $L_{2}(8) \cong E\left(C_{G_{0}}(e)\right) \leqslant D_{0}$ for each $e \in E_{0}-\langle z\rangle$. One can now use (2.8) to conclude that $D_{0}=G_{0}$.

(5.14) Let $G_{0}={ }^{2} F_{4}(q)^{\prime}$. Then one of the following holds:

(i) $q>2$ and $D_{0}=G_{0}$.

(ii) $q=2, p=5, E_{0} \cong Z_{5} \times Z_{5}$, and $C_{G_{0}}^{0}\left(E_{0}\right) \leqslant N_{G_{0}}\left(E_{0}\right)$.

(iii) $q=2, p=3, E_{0} \cong Z_{3} \times Z_{3}$, and $C_{G_{0}}^{0}\left(E_{0}\right) \cong L_{3}(3)\langle\gamma\rangle$, where $\gamma$ is the graph automorphism of $L_{3}(3)$. In this case if we set $G_{1}={ }^{2} F_{4}(2)$, then $C_{G_{1}}^{0}\left(E_{0}\right)=G_{1}$.

Proof. By (5.3) we may assume that $p$ is associated with $\Phi_{1}, \Phi_{2}$, or $\Phi_{4}$. Suppose the latter holds and $q \neq 2$. Then $G_{0}$ contains a subgroup $X=\left(X_{1} \times X_{2}\right)\langle t\rangle$, where $X_{2}=X_{1}^{t}, X_{1} \cong S z(q)$, and $t^{2}=1$. By (5.2) we may take $E_{0} \leqslant X_{1} \times X_{2}$ and $E_{0}=\Omega_{1}(P)$ for $P \in \operatorname{Syl}_{p}\left(G_{0}\right)$. Clearly $\left(X_{1} \times X_{2}\right)\langle t\rangle \leqslant D_{0}$. However the arguments used in (5.8) do not work here because of the lack of root involutions. We proceed as follows.

Let $S_{1} \in \operatorname{Syl}_{2}\left(X_{1}\right)$ and set $S_{2}=S_{1}^{t}$. Choose $1 \neq x \in \Omega_{1}\left(S_{1}\right)$. Then $S_{2} \leqslant C_{G_{0}}(x)$ so $x$ is 2-central in $G_{0}$ (see e.g. (18.6) of [3]). Now $C_{D_{0}}(x) \geqslant S_{1} \times X_{2}$ and $C_{G_{0}}(x)=O^{2^{\prime}}(P)$ for $P$ a maximal parabolic subgroup of $G_{0}$. The structure of $C_{G_{0}}(x)$ is given explicitly in $\$ 10$ of [12]. We check that if $C_{D_{0}}(x)>S_{1} \times X_{2}$, then $C_{D_{0}}(x) \geqslant S_{1} \cdot O_{2}(P)^{\prime}$, and it is easy to check that this group and $X_{1}$ generate $G_{0}$. So suppose $C_{D_{0}}(x)=S_{1} \times X_{2}$. Let $S=\left(S_{1} \times S_{2}\right)\langle t\rangle$. Clearly $Z\left(S_{1}\right) \times Z\left(S_{2}\right)$ is weakly closed in $S$, and hence $S_{1} \times S_{2}=C_{S}\left(Z\left(S_{1}\right) Z\left(S_{2}\right)\right)$ is characteristic in $S$. By the Krull-Schmidt theorem (p. 120 of [16]) the pair $\left\{S_{1} Z\left(S_{2}\right), Z\left(S_{1}\right) S_{2}\right\}$ is characteristic in $S$, and taking squares, we conclude that $\left\{Z\left(S_{1}\right), Z\left(S_{2}\right)\right\}$ is characteristic in $S$. From this and the above we see that $S \in \operatorname{Syl}_{2}\left(D_{0}\right)$. Also Corollary 4 of [13] shows that $Z\left(S_{1}\right) Z\left(S_{2}\right)$ is strongly closed in $S$. But now consider $C_{X_{1} X_{2}}(t) \cong S z(q)$. By (18.6) of [3] $t$ is $G_{0}$-conjugate to an involution in $Z\left(S_{1}\right)$. Applying Sylow's theorem in $C_{G_{0}}(t)$ to $\langle e\rangle=E \cap C_{X_{1} X_{2}}(t)$ we see that $Y=O^{2^{\prime}}\left(C_{G}(e)\right) \cong S z(q)$. But then $t \in Y \leqslant D_{0}$ and $t$ is a square in $D_{0}$. This contradicts the fact that $Z\left(S_{1}\right) Z\left(S_{2}\right)$ is strongly closed in $S$. So $D_{0}=G_{0}$ in this case.

If $q=2$ and $p=5$, then $E_{0}=O_{5}\left(X_{1} X_{2}\right)$ (notation as above) and $E_{0} \in \operatorname{Syl}_{5}\left(G_{0}\right)$. If $E_{i}=O_{5}\left(X_{i}\right)$, then $C_{G_{0}}\left(E_{i}\right)=E_{i} \times X_{j}$, where $\{i, j\}=\{1,2\}$. Also it is easy to use 
the above arguments to check that $N_{G_{0}}\left(E_{0}\right)$ is transitive on $E_{0}^{\#}$. It follows that $C_{G_{0}}^{0}\left(E_{0}\right) \leqslant N\left(E_{0}\right)$ and (ii) holds.

We now suppose $p \mid q \pm 1$. If $p \mid q-1$, then by (5.2) $E_{0}^{g} \leqslant H$ for some $g \in G$, and so (i) holds by (2.3). So, assume $p \mid q+1$. Suppose $p>3$. Then by (5.2) we may assume

$$
E_{0} \leqslant\left\langle U_{ \pm \alpha_{1}}\right\rangle \times\left\langle U_{ \pm \alpha_{1}}^{s_{2} s_{1} s_{2}}\right\rangle \cong S L(2, q) \times S L(2, q)
$$

This implies $E_{0} \leqslant C\left(E_{1}\right)$ where $E_{1} \cong Z_{3} \times Z_{3}$. If $x \in E_{1}^{\#}$, then by [7] $C_{G_{0}}(x) \cong S U(3, q)$. By (4.1) it will suffice to show that $G_{0}=C_{G_{0}}^{0}\left(E_{1}\right)$. That is, we may now assume $p=3$.

Next we will show that it suffices to look at the case $q=2$. First note that ${ }^{2} F_{4}(2)$ is a subgroup of ${ }^{2} F_{4}(q)$. Choose $E_{0} \leqslant\left\langle U_{ \pm \alpha_{1}}\right\rangle \times\left\langle U_{ \pm \alpha_{1}}^{s_{2} s_{1} s_{2}}\right\rangle=X$. With the natural embedding of $Y={ }^{2} F_{4}(2) \leqslant G_{0}$ we may take $E_{0} \leqslant{ }^{2} F_{4}(2)^{\prime}$. Each involution in ${ }^{2} F_{4}(2)$ is in ${ }^{2} F_{4}(2)^{\prime}$ (see 18.6 of [3]). Since the Weyl group of ${ }^{2} F_{4}(2)$ and $G_{0}$ are the same, we may consider the Weyl group of $G_{0}$ as a subgroup of $Y$. Let $I=\langle X, Y\rangle \leqslant D_{0}$. Then $I$ contains all subgroups $\left\langle U_{ \pm \alpha}\right\rangle$ of $G_{0}$ for $U_{\alpha} \sim U_{\alpha_{1}}$. From the commutator relations (see $\S 10$ of [12]) we see that if $\beta$ is any root with $\left\langle U_{ \pm \beta}\right\rangle \cong S z(q)$, then $\Omega_{1}\left(U_{\beta}\right) \leqslant I$. For such a root $\beta$ we have $I \cap\left\langle U_{ \pm \beta}\right\rangle \geqslant\left\langle\Omega_{1}\left(U_{ \pm \beta}\right)\right\rangle=\left\langle U_{ \pm \beta}\right\rangle$, for $\left.q\right\rangle 2$. So $I=G_{0}$ and it suffices to look at the case $q=2$.

Now $G_{0}={ }^{2} F_{4}(2)^{\prime}, E_{0} \cong Z_{3} \times Z_{3}$, and by Burgoyne [7] each $e \in E_{0}^{\#}$ satisfies $C(e) \cap{ }^{2} F_{4}(2) \cong S U(3,2)$. We then have $C_{G_{0}}(e)$ a subgroup of index 2 in $S U(3,2)$. Write $E_{0}=\langle a\rangle \times\langle b\rangle$. In $G_{1}={ }^{2} F_{4}(2)$ there is just one class of such subgroups and it follows that $G_{0} \geqslant X_{1} \times X_{2} \cong S_{3} \times S_{3}$, with $\langle a\rangle=O_{3}\left(X_{1}\right)$ and $\langle b\rangle=O_{3}\left(X_{2}\right)$. So $C_{G_{0}}(a)$ contains an involution, $t_{1}$, that inverts $b$. Let $S=O_{3}\left(C_{G_{1}}(b)\right)$. We now have $N_{G_{1}}(\langle b\rangle)$ an extension of $S$ by a semidihedral group of order 16 , and $N_{G_{0}}(\langle b\rangle)$ an extension of $S$ by $D_{8}$. Let $T$ be a Sylow 2-subgroup of $N_{G_{0}}(\langle b\rangle)$, with $t_{1} \in T$. Then $E_{0}^{T}=\left\{E_{0}, E_{1}\right\}$ where $E_{1}=\langle b, c\rangle$ and $E_{0} E_{1}=S . T$ contains a klein group $T_{0}=\left\langle t_{1}\right\rangle \times\left\langle t_{2}\right\rangle$ with the following properties: $t_{1}$ inverts $c, t_{2}$ centralizes $c, t_{2}$ inverts $a$, and $t_{2}$ inverts $b$. So $T_{0}=N_{T}\left(E_{0}\right)=N_{T}\left(E_{1}\right)$ and $T=T_{0}\langle j\rangle$, where $j$ is an involution with $t_{i}^{j}=t_{2}$ and $a^{j}=c$.

Let $P_{0}=N_{G_{0}}\left(E_{0}\right)$ and $P_{1}=N_{G_{0}}\left(E_{1}\right)$. Looking at $P_{0} \cap C(a)$ and $P_{0} \cap C(b)$ we easily have $P_{0}$ being the semidirect product of $E_{0}$ with $G L(2,3)$. Similarly for $P_{1}$. Set $X=\left\langle P_{0}, P_{1}\right\rangle$. Then $X=X^{j}$ and we claim that $X \cong L_{3}(3)$.

The claim is proved by first giving a $(B, N)$-pair in $X$. Let $Y=P_{0} \cap P_{1}=S\left\langle t_{1}, t_{2}\right\rangle=S T_{0}$. $Y$ will be a Borel subgroup of $X$ and $T_{0}$ a Cartan subgroup. $P_{0}$ is 2-transitive on the cosets of $Y$, so there is an involution $j_{0} \in N_{P_{0}}\left(T_{0}\right)$ such that $P_{0}=Y \cup Y j_{0} Y$. Similarly $P_{1}=Y \cup Y j_{1} Y$, where we may take $j_{1}=j_{0}^{j}$. Now $S=\langle a\rangle\langle b\rangle\langle c\rangle$ and we then have

$$
\begin{aligned}
j_{0} S j_{1} & =j_{0}\langle a\rangle\langle b\rangle\langle c\rangle j_{1}=(\langle a\rangle\langle b\rangle)^{j_{0}} j_{0} j_{1}\langle c\rangle^{j_{1}} \\
& \subseteq\langle a, b\rangle j_{0} j_{1}\langle b, c\rangle \subseteq S j_{0} j_{1} S .
\end{aligned}
$$

This implies $j_{0} Y j_{1} \subseteq Y j_{0} j_{1} Y$. To get additional relations observe that since $j_{0}$ normalizes $T_{0}, j_{0}$ must permute the proper subgroups of $E_{0}$ that are $T_{0}$-invariant, namely $\langle a\rangle$ and $\langle b\rangle$. As $j_{0} \notin N(\langle b\rangle), j_{0}$ interchanges $\langle a\rangle$ and $\langle b\rangle$. Similarly $j_{1}$ 
interchanges $\langle b\rangle$ and $\langle c\rangle$. Thus,

$$
\begin{aligned}
j_{0} S j_{1} j_{0} & =j_{0} E_{0}\langle c\rangle j_{1} j_{0}=E_{0} j_{0}\langle c\rangle j_{1} j_{0}=E_{0} j_{0} j_{1}\langle b\rangle j_{0} \\
& =E_{0} j_{0} j_{1} j_{0}\langle a\rangle \subseteq S j_{0} j_{1} j_{0} S .
\end{aligned}
$$

At this point the additional relations are obtained by proving that $\left\langle j_{0}, j_{1}\right\rangle \cong S_{3}$. Now $\left\langle j_{0}, j_{1}\right\rangle$ induces $S_{3}$ on $\left\langle t_{1}, t_{2}\right\rangle$ and $\left\langle j_{0}, j_{1}\right\rangle \leqslant\left\langle j_{0}, j\right\rangle$ as a subgroup of index 2 . From the known information on centralizers of elements of order 3 and 5 we see that if $\left\langle j_{0}, j_{1}\right\rangle \not S_{3}$, then $\left\langle j_{0}, j\right\rangle$ is dihedral of order divisible by 24 , and an element of order 4 centralizes $\left\langle t_{1}, t_{2}\right\rangle$. To see that this is impossible argue as follows. We may take $X_{1}=\left\langle U_{ \pm \alpha_{1}}\right\rangle$ and $X_{2}=\left\langle U_{ \pm \alpha_{1}}^{s_{2} s_{1} s_{2}}\right\rangle$. Then we have $\left\langle t_{1}, t_{2}\right\rangle \sim\left\langle U_{\alpha_{1}}, U_{\alpha_{1}}^{s_{2} s_{1} s_{2}}\right\rangle$, and using (18.6)(ii) of [3] together with the relations in $\S 10$ of [12], we see that $C\left(\left\langle t_{1}, t_{2}\right\rangle\right)$ is elementary abelian. So $\left\langle j_{0}, j_{1}\right\rangle \cong S_{3}$ and $X \cong L_{3}(3)$ by Theorem 2 of [15]. Thus $X\langle j\rangle \cong \operatorname{Aut}\left(L_{3}(3)\right)$.

From the structure of $\operatorname{Aut}\left(L_{3}(3)\right)$ we see that $C_{G_{0}}(e) \leqslant X\langle j\rangle$ for each $e \in E_{0}^{\#}$. Also $C_{X}^{0}\left(E_{0}\right)$ is a maximal parabolic subgroup of $X$. Indeed $C_{X}^{0}\left(E_{0}\right)=N_{X}\left(E_{0}\right)$. Since $C_{X\langle j\rangle}(b)>C_{X}(b)$ we have $C_{G_{0}}^{0}\left(E_{0}\right)=C_{G_{0}}^{0}\left(E_{1}\right)=X\langle j\rangle$. So $D_{0}=X\langle j\rangle$ for this choice of $E_{0}$. Now, in $G_{1}={ }^{2} F_{4}(2)$ there is just one class of subgroups of type $Z_{3} \times Z_{3}$. So $C_{G_{0}}^{0}\left(E_{0}\right) \cong \operatorname{Aut}\left(L_{3}(3)\right)$ for any such subgroup $E_{0} \leqslant G_{0}$.

If $D_{1}=C_{G_{1}}^{0}\left(E_{0}\right)$ for $E_{0}$ as above, then we easily see that $D_{1}$ is transitive on elements of order 3 in $D_{1}$ and $D_{1}$ is strongly 3 -embedded in $G_{1}$. So $\left|G_{1}: D_{1}\right| \equiv 1$ $(\bmod 27)$. But with $D_{0}<D_{1}$ this is impossible. The proof of $(5.14)$ is now complete.

With the results of this section we have now completed the proof of Theorem 1 .

6. Automorphisms and Theorem 2. In this section we deal with the case $G$ simple, $E \leqslant \operatorname{Aut}(G), E \cong E_{p^{n}}$ with $n>1$, but $E$ not contained in the subgroup of $\operatorname{Aut}(G)$ generated by inner and diagonal automorphisms of $G$. Since $p$ is odd this usually means that some element of $E$ induces a field automorphism of $G$. Let $G_{0}=\hat{G}_{\sigma}$ and let $G_{1}$ be the inverse image in $\hat{G}$ of $\bar{G}_{\sigma}$. Then $G_{1} / Z\left(G_{1}\right)$ is isomorphic to $G$ extended by all diagonal automorphisms of $G$. Let $G_{2}$ be the extension of $G_{1}$ by field automorphisms of $G_{0}$.

We first assume that $E$ is contained in $G_{2} / Z\left(G_{0}\right)$. There is a $p$-subgroup $E_{0} \leqslant G_{2}$ such that $E_{0} Z\left(G_{0}\right) / Z\left(G_{0}\right)=E$. Set $D_{0}=C_{G_{0}}^{0}\left(E_{0}\right)$ and $D=C_{G}^{0}(E)$. Then $D_{0}=G_{0}$ implies that $D=G$. Note that the only cases not included under the above restriction on $E_{0}$ is $G \cong D_{4}(q)$ or ${ }^{3} D_{4}(q)$.

(6.1) Suppose $E_{0} \leqslant G_{2}$, but $E_{0} \neq G_{1}$.

(i) If $p \geqslant 7$, then $D_{0}=G_{0}$.

(ii) Suppose $m_{p}(E)>2$ and $D_{0}<G_{0}$. For $e \in E_{0}-\left(E_{0} \cap G_{1}\right)$ set $L=C_{G_{0}}(e)$ and $E_{1}=E_{0} \cap G_{1}$. Then there exists $e$ such that $C_{L}^{0}\left(E_{1}\right)<L$. So the pair $(L, p)$ is given in Theorem 1 .

(iii) If $m_{p}(E)=2$, either $D_{0}=G_{0}$, or $p=5$ and $G \cong S z\left(2^{5}\right)$, or $p=3$ and $G \cong L_{2}(8)$.

Proof. Suppose $D_{0}<G_{0}$. By Lang's theorem [18] each element in $E_{0}-\left(E_{0} \cap G_{1}\right)$ induces a field automorphism on $G_{0}$. Set $E_{1}=E_{0} \cap G_{1}$. If $m_{p}(E)=2$, then $C_{G_{0}}(e) \leqslant D_{0}$ for each $e \in E_{0}-E_{1}$. Suppose $m_{p}(E)>2$. If (ii) fails, then $C_{G_{0}}(e) \leqslant D_{0}$ for each $e \in E_{0}-E_{1}$. So in all cases we have $C_{G_{0}}(e) \leqslant D_{0}$ for each $e \in E_{0}-E_{1}$. 
First suppose that for $e \in E_{0}-E_{1}, C_{G_{0}}(e) \neq S_{3}, A_{4}$, or $S z(2)$. Then Theorem 1 of [8] implies that $O^{r^{\prime}}\left(C_{G}(e)\right) \leqslant D \leqslant C_{G}(e)$ for each $e \in E_{0}-E_{1}$ (we are using the fact that $\left.O^{r^{\prime}}\left(C_{G}(e)\right)=C_{G_{0}}(e) Z\left(G_{0}\right) / Z\left(G_{0}\right)\right)$. Since $E$ is generated by such elements $e$, we have $O^{r^{\prime}}\left(C_{G}(e)\right) \leqslant C_{G}(E)$ for $e \in E_{0}-E_{1}$. This contradicts (2.3).

Consider the three exceptional cases. If $O^{r^{\prime}}\left(C_{G}(e)\right) \cong S_{3}$, then $p=3$ and $G \cong L_{2}(8)$. If $O^{r^{\prime}}\left(C_{G}(e)\right) \cong S z(2)$, then $p=5$ and $G \cong S z\left(2^{5}\right)$. For these cases (iii) holds. Finally, assume $O^{r^{\prime}}\left(C_{G}(e)\right) \cong A_{4}$. Here $p=3=r$, against our standing hypothesis. We have now proved (6.1).

The pairs $(G, p)$ appearing in (i), (ii), and (iv) of Theorem 1 each give rise to larger configurations where generation fails. These correspond to the situation of (6.1)(ii).

(6.2) Suppose $\left(G_{0}, E_{0}, p\right)$ is one of the cases constructed in (4.1) or (5.1) where generation fails, and suppose that the pair $(G, p)$ satisfies (i), (ii), or (iv) of Theorem 1. Let $G_{0}=\hat{G}_{\sigma}, \tilde{G}_{0}=\hat{G}_{\sigma^{p}}$ and $\tilde{E}_{0}=E_{0}\langle\tau\rangle$, where $\tau$ is the field automorphism of order $p$ that $\sigma$ induces on $\tilde{G}_{0}$. Then $C_{\tilde{G}_{0}}^{\ell}\left(\tilde{E}_{0}\right)<\tilde{G}_{0}$.

Proof. We are assuming that $G_{0}$ is one of the groups $O^{ \pm}(2 n, 2)^{\prime}, S p(2 n, 2)$, $S U(n, 2), E_{6}(2),{ }^{2} E_{6}(2), F_{4}(2), E_{7}(2), E_{8}(2), G_{2}(2)^{\prime}$, or ${ }^{2} F_{4}(2)^{\prime}$. In the last case $p=5$; otherwise $p=3$. Let $E_{0}$ be as constructed in the proof of (4.1) or (5.1). In each of these cases it is clear from the definition of $E_{0}$ that $\tilde{G}_{0}$ contains a subgroup $I$ such that $I$ is a $\tau$-invariant homocyclic $p$-group satisfying $E_{0}=\Omega_{1}(I)=[I, \tau]$. For example, in many of these cases $E_{0}=O_{3}\left(J_{1} \times \cdots \times J_{k}\right)$, where each $J_{i} \cong S L(2,2)$ and each $J_{i}$ is generated by conjugates of long root subgroups. Here we take $E_{0}<I$ with $I \in \operatorname{Syl}_{3}\left(\tilde{J}_{1} \times \cdots \tilde{J}_{k}\right)$, where $J_{i}<\tilde{J}_{i} \cong S L\left(2,2^{3}\right)$ and $\tilde{J}_{i}$ is generated by long root subgroups of $\tilde{G}_{0}$.

For the moment exclude the configuration of (4.1)(iii). Then in each of the remaining cases we showed that $C_{G_{0}}^{0}\left(E_{0}\right) \leqslant N_{G_{0}}\left(E_{0}\right)$, and we claim that $C_{\tilde{G}_{0}}^{0}\left(\tilde{E}_{0}\right)<$ $N_{\tilde{G}_{0}}\left(E_{0}\right)$. To see this let $F$ be a maximal subgroup of $\tilde{E}_{0}$. If $F=E_{0}$, then clearly $C(F) \leqslant N\left(E_{0}\right)$. Suppose $F \neq E_{0}$, so $F=F_{1}\langle e \tau\rangle$, where $F_{1}=F \cap E_{0}$ and $e \in E_{0}$. There exists $g \in I$ such that $\tau^{g}=e \tau$. Then $C_{\tilde{G}_{0}}(F)=$ $C_{\tilde{G}_{0}}\left(F_{1}\langle e \tau\rangle\right)=C_{\tilde{G}_{0}}\left(F_{1}\langle\tau\rangle\right)^{g}=C_{G_{0}}(F)^{g} \leqslant N_{G_{0}}\left(E_{0}\right)^{g} \leqslant N_{\tilde{G}_{0}}\left(E_{0}\right)$. This proves the claim.

If (4.1)(iii) holds, then $G_{0} \cong S p(2 n, 2), \tilde{G}_{0} \cong S p(2 n, 8)$, and $D_{0} \cong O^{ \pm}(2 n, 2)$. Here embed $D_{0}$ in $\tilde{D}_{0} \cong O^{ \pm}(2 n, 8)$, such that $\tilde{D}_{0}^{\tau}=\tilde{D}_{0}$. We can then choose $I<\tilde{D}_{0}$ and use the above argument to get $C_{G_{0}}^{0}\left(\tilde{E}_{0}\right) \leqslant \tilde{D}_{0}$.

(6.3) Suppose (6.1)(ii) holds with the pair $(L, p)$ given in Theorem 1(iii). Then $D_{0}=G_{0}$.

Proof. We have $p=3$ and $L \cong S L\left(3^{k}, 4\right),{ }^{2} F_{4}(2)^{\prime}, F_{4}(4), \hat{E}_{6}(4)$, or $E_{8}(4)$. By (6.1)(iii) we may take $m_{p}(E) \geqslant 3$. The case $L \cong{ }^{2} F_{4}(2)^{\prime}$ is out since $L=C_{G_{0}}(e)$ (see (5.14)(iii)). Let $E_{1}=E_{0} \cap G_{1}$.

First assume $L \cong S L(3,4)$, so $G_{0} \cong S L(3,64)$. By (4.1) we may write $E_{1}=\langle a, b\rangle$ where $[a, b]=z$ and $\langle z\rangle=Z\left(G_{0}\right)$. Write $E_{0}=E_{1}\langle e\rangle$ and choose $e$ so that $[a, e]=z$. Then $T=C_{G_{0}}(e) \cong S L(3,4)$ and $a$ induces an outer automorphism of order 3 on $T$. Then $C_{T}(a) \cong Z_{21}$ as otherwise $C_{T}(a) \cong Z_{3} \times L_{2}(4)$ and $E_{1}$ normalizes a 2-group $L$, contradicting (2.3). Thus, $C_{G_{0}}(F)=C_{T}(a) \cong Z_{21}$ where $F=\langle a, e, z\rangle$. Now letting $a$ vary we conclude that $T \leqslant C_{G_{0}}^{0}\left(E_{0}\right)$. Next, let $e$ vary to conclude that $D_{0}=G_{0}$. 
Suppose $L \cong S L\left(3^{k}, 4\right)$ with $k>1$. Let $E_{0}=E_{1}\langle e\rangle$ and choose $a, b \in E_{1}$ such that $\langle a\rangle Z\left(G_{0}\right) \neq\langle b\rangle Z\left(G_{0}\right)$. Then consideration of the usual module for $G_{0}$ shows that $\langle a, b\rangle$ normalizes a proper parabolic subgroup of $G_{0}$. By $(2.3) C_{G_{0}}^{r}(\langle a, b\rangle)=G_{0}$. Now (2.16), Theorem 1, (6.1), induction and the above paragraph yield $D_{0}=G_{0}$. Essentially the same argument works for the cases $L=F_{4}(4), \hat{E}_{6}(4)$, or $E_{8}(4)$. Namely, choose $\langle a, b\rangle \leqslant E_{1}$ with $\langle a\rangle Z\left(G_{0}\right) \neq\langle b\rangle Z\left(G_{0}\right)$ and use [7] to conclude that $\langle a, b\rangle$ stabilizes a proper parabolic subgroup of $G_{0}$. Then proceed as before. This proves (6.3).

We will have completed the proof of Theorem 2 once we deal with the case $E_{0} \neq G_{2}$. This requires the existence of a graph automorphism of order 3 , so $G_{0} \cong D_{4}(q)$ or ${ }^{3} D_{4}(q)$.

(6.4) Suppose $G_{0} \cong{ }^{3} D_{4}(q)$ and $E_{0} \$ G_{2}$. Then either $C_{G_{0}}^{0}\left(E_{0}\right)=G_{0}$ or $q=2$, $p=3$ and there exists $E_{0} \cong Z_{3} \times Z_{3} \times Z_{3}$ such that $C_{G_{0}}^{0}\left(E_{0}\right)<G_{0}$.

Proof. First suppose $q>2$ and note that $E_{0} \leqslant G_{0}\langle\tau\rangle$, where $\tau$ denotes the graph automorphism of $D_{4}\left(q^{3}\right)$, restricted to $G_{0}$. Then $G_{2}(q) \cong C_{G_{0}}(\tau)$. By Table (3.3) $C_{G_{0}}(\tau)$ contains a group $L \cong S L(3, q)$ or $S U(3, q)$, according to $3 \mid q-1$ or $3 \mid q+1$.

Let $S \in \operatorname{Syl}_{3}(L)$. By Table (5.2) $S$ has index 3 in a Sylow 3-subgroup of $G_{0}$, so $S \times\langle\tau\rangle$ has index 3 in a Sylow 3-subgroup of $G_{0}\langle\tau\rangle$. Therefore, we may assume that $E_{0} \leqslant N(S \times\langle\tau\rangle) \leqslant N(Z(S \times\langle\tau\rangle))=N(\langle x, \tau\rangle)$, where $\langle x\rangle=Z(L)=Z(S)$. Since $C_{G_{0}}(\tau)$ does not contain a Sylow 3-subgroup of $G_{0}, \tau \sim \tau x^{i}$ for $i=0,1,2$. Now apply Theorem 2 of [8] to conclude that $G_{0}=\left\langle O^{r^{\prime}}\left(C_{G_{0}}\left(\tau x^{i}\right)\right) \mid i=0,1,2\right\rangle$. So if $E_{0} \leqslant C(\langle x, \tau\rangle)$, then $G_{0}=C_{G_{0}}^{0}\left(E_{0}\right)$ by Theorem 1 . Hence we may assume $E_{0} \$ C(\tau)$.

By (2.9) we may assume $x \in E_{0}$. Suppose $q>4$. Then $L \leqslant D_{0}$ so $L \leqslant\left\langle V^{G} \cap D_{0}\right\rangle$, where $V$ is a long root subgroup of $G_{0}$. Using (2.8) and Table (5.2) we conclude that $E\left(D_{0}\right)=L, D_{0}=G_{0}$, or $E\left(D_{0}\right) \cong G_{2}(q)$. Suppose $E\left(D_{0}\right)=G_{2}(q)$. Then one checks that $O^{r^{\prime}}\left(D_{0} E_{0}\right) \cong G_{2}(q) \times Z_{3}$, the $Z_{3}$ factor being generated by a conjugate of $\tau$. By (2.9) we may assume $\tau^{G} \cap E_{0} \neq \varnothing$, which is not the case. Suppose $E\left(D_{0}\right)=L$. For $e \in E_{0}-\langle x\rangle$ one can argue within $\hat{G}$ to conclude that $O^{r^{\prime}}\left(C_{G_{0}}(e)\right) \neq 1$. But $q>4$ implies $O^{r^{\prime}}\left(C_{G_{0}}(e)\right) \leqslant D_{0} \leqslant N(L)$. Since $L=E\left(N_{G_{0}}(L)\right), O^{r^{\prime}}\left(C_{G_{0}}(e)\right) \leqslant L$. In particular $\langle e, x\rangle$ centralizes a nontrivial $r$-subgroup of $G$. By $(2.3), C_{G_{0}}^{r}(\langle e, x\rangle)=G_{0}$, while the above arguments applied to $e_{1} \in\langle e, x\rangle$ show $C_{G_{0}}^{r}(\langle e, x\rangle) \leqslant N(L)$. This is a contradiction. Hence $D_{0}=G_{0}$.

Next, suppose $q=4$. Then $L \cong S L(3,4)$. If $m_{3}\left(E_{0}\right)=2$, then $L=O^{r^{\prime}}(C(x)) \leqslant D_{0}$ and we argue as above to get $D_{0}=G_{0}$. Suppose $m_{3}\left(E_{0}\right) \geqslant 3$. Then $m_{3}\left(E_{0}\right)=3$ and we write $E_{0}=\langle x, e, a \tau\rangle$ for some $e \in G_{0}$ such that $\tau^{e} \neq \tau$. Since $S\langle\tau\rangle=S \times\langle\tau\rangle$ we may take $a \in S$. Then $e \notin S \times\langle\tau\rangle$. Now, $C_{L}(e) \cong Z_{3} \times L_{2}(4)$ or $Z_{21}$. In the former case, $E_{0}$ normalizes a proper 2-subgroup of $G_{0}$, so (2.3) implies $D_{0}=G_{0}$. Assume $C_{L}(e) \cong Z_{21}$. Then $L=\left\langle C_{I}(v): v \in\langle e, a \tau\rangle-\langle a \tau\rangle\right\rangle$, so $L \leqslant$ $C_{G_{0}}^{0}\left(E_{0}\right)=D_{0}$. As above, either $D_{0}=G_{0}$ or $L \unlhd D_{0}$. So suppose $L \unlhd D_{0}$. Let $y \in\langle x, e\rangle^{\#}$. By [7] either $O^{r^{\prime}}(C(y)) \cong L_{2}(64)$ or $y \sim x$. In the former case we have $O^{r^{\prime}}(C(y)) \leqslant D_{0}$, against $L=E\left(D_{0}\right)$. So $\langle x, e\rangle^{\#}$ is fused in $G_{0}$. In particular, $E\left(C_{G_{0}}(e)\right) \cong S L(3,4)$. Since $C(e) \cap C(x)$ contains 7-elements, $x$ induces an outer automorphism on $E\left(C_{G_{0}}(e)\right)$ and we can then argue that $E\left(C_{G_{0}}(e)\right) \leqslant D_{0} \leqslant N(L)$, a contradiction. 
Finally, let $q=2$ and retain the above notation. Set $E_{0}=\langle x, a, \tau\rangle$, where $a \in S-\langle x\rangle$. Then $S \unlhd C_{G_{0}}(x)$, as $L \cong S U(3,2)$, so $S \unlhd C_{G_{0}}(F)$ for any hyperplane, $F$, of $E_{0}$ with $x \in F$. Suppose $F$ is a hyperplane of $E_{0}$ with $F \cap S=\left\langle a x^{j}\right\rangle$ for $j=0,1$, or 2 . Then $\tau x^{i} \in F$ for some $i=0,1,2$, and $\tau \sim \tau x^{i}$ implies that $C_{G_{0}}(\tau) \cong G_{2}(2) \cong \operatorname{Aut}\left(U_{3}(3)\right)$. Now $S \in \operatorname{Syl}_{3}\left(C_{G_{0}}\left(\tau x^{i}\right)\right)$ and it follows that $C_{G_{0}}(F) \leqslant N_{G_{0}}(S)$. We have now proved $C_{G_{0}}^{0}\left(E_{0}\right) \leqslant N_{G_{0}}(S)$, so (6.4) is proved.

Similar arguments will be used for $G_{0}=D_{4}(q)$.

(6.5) Suppose $G=D_{4}(q)$ and $E_{0} \$ G_{2}$. Then one of the following holds:

(i) $C_{G_{0}}^{0}\left(E_{0}\right)=G_{0}$.

(ii) $q=2$ or $4, p=3$, and $E_{0} \leqslant D_{4}(q)\langle\sigma\rangle$ for $\sigma$ a graph automorphism (of order 3) of $G_{0}$.

(iii) $q=8$ and $E_{0} G_{0}=O^{3^{\prime}}\left(\operatorname{Aut}\left(D_{4}(8)\right)\right)$.

Moreover if $q=2,4$ or 8 , there exists $E_{0}<\operatorname{Aut}\left(G_{0}\right)$ for which $C_{G_{0}}^{0}\left(E_{0}\right) \leqslant N_{G_{0}}\left(E_{0}\right)$.

Proof. Suppose $E_{0}$ contains an element in the coset of $G_{1}$ given by a field or graph-field automorphism. Then we can argue as in (6.1) to get $C_{G_{0}}^{0}\left(E_{0}\right)=G_{0}$, except when $q=8$ and (iii) holds or $E_{0} \cap G_{0}=1$. Suppose $E_{0} \cap G_{0}=1$. Then $E_{0} \cong Z_{3} \times Z_{3}$ and by Lang's theorem $E_{0}$ contains both a field automorphism and a graph-field automorphism of $G_{0}$. Here $C_{G_{0}}^{0}\left(E_{0}\right)=G_{0}$ by Theorem 1 of [8]. Suppose $q=8$. Let $\sigma$ be a field automorphism of $G_{0}$ of order $3, \tau$ a graph automorphism of order 3 , and $[\sigma, \tau]=1$. Then $C_{G_{0}}(\sigma) \cap C_{G_{0}}(\tau) \cong G_{2}(2)$ and we let $E_{1}$ be an elementary subgroup of $C_{G_{0}}(\langle\sigma, \tau\rangle)$ of order 9. Set $E_{0}=E_{1}\langle\sigma, \tau\rangle$.

Let $J=J_{1} \times J_{2} \times J_{3} \times J_{4}$ with each $J_{i}$ generated by opposite root subgroups of $G_{0}$. We may assume that $\sigma$ induces a field automorphism on each $J_{i}$ and that $\langle\tau\rangle$ is transitive on $\left\{J_{1}, J_{2}, J_{3}\right\}$ while fixing $J_{4}$. Let $E_{0}=\langle a, b\rangle$ with $a \in J_{4}$ and $b \in J_{1} J_{2} J_{3}$. We claim $C_{G_{0}}^{0}\left(E_{0}\right) \leqslant N(\langle b\rangle)$. Let $F$ be a hyperplane in $E_{0}$. If $b \in F$, then clearly $C_{G_{0}}(F) \leqslant N(\langle b\rangle)$, so suppose $b \notin F$. Since $a, a b, a b^{-1}$ are all conjugate in $C_{G_{0}}(\sigma) \cap C_{G_{0}}(\tau) \cap N\left(E_{0}\right)$, we may assume $a \in F$. From here the claim is easily checked.

From now on we assume $E_{1}=E \cap G_{0}$ is a hyperplane of $E_{0}$ and $E_{0} \leqslant G_{1}\langle\tau\rangle$ for $\tau$ a graph automorphism of $G_{0}$ such that $|\tau|=3$. From Table (3.3) and its verification we conclude that $G_{0}$ contains a subgroup $X$ such that there exists $L \unlhd X$ with $L \cong S L(3, q)$ or $S U(3, q)$, according to $q \equiv 1,-1(\bmod 3)$. Also, $Z=Z(X) \cong Z_{q \mp 1} \times Z_{q \mp 1}$ and $|X: L Z|=3$. Finally, $X^{\tau}=X$ and $L=C_{X}(\tau)$. By Table (5.2) we may assume $E_{0} \leqslant X\langle\tau\rangle$ and by (2.9) we may assume $x \in E_{1}$, where $\langle x\rangle=Z(L)$. Assume $q>4$. Then (4.1) implies $L \leqslant C_{G_{0}}^{0}\left(E_{0}\right)$.

Suppose $\tau \in E_{0}$. Then $E_{1} \leqslant C_{G_{0}}(\tau) \cong G_{2}(q)$, and so $E_{1}$ is contained in a Sylow 3-subgroup of $C_{G_{0}}(\tau)$. Table (5.2) implies that $L$ contains a Sylow 3-subgroup of $C_{G_{0}}(\tau)$, so assume $E_{1}<L$. Therefore $Z(X) \leqslant C\left(E_{1}\right) \leqslant C_{G_{0}}^{0}\left(E_{0}\right)$. By (5.1) $G_{2}(q) \cong C_{G_{0}}(\tau) \leqslant C_{G_{0}}^{0}\left(E_{0}\right)$ and so $C_{G_{0}}^{0}\left(E_{0}\right) \geqslant\left\langle C_{G_{0}}(\tau), Z\right\rangle=G_{0}$ (use (2.8) and the fact that $C_{G_{0}}(\tau)$ is self-normalizing in $G_{0}$ ). From now on assume $E_{0}$ contains no conjugate of $\tau$.

The results in [9] (see (3.5)) imply that we may assume $(\tau a)^{g} \in E_{0}$, where $a \neq 1$ and $a \in H \cap\left\langle U_{ \pm \alpha_{2}}\right\rangle$. Also, using the Bruhat decomposition we see that $C_{\hat{G}}(\tau a)^{g} \cong P S L(3, K)$ (or see (4.3) of [9]) and then $C_{G_{0}}(\tau a)^{g}=C_{\hat{G}_{0}}(\tau a)^{g} \cong P G L(3, q)$ 
or $P G U(3, q)$, according to $q \equiv 1,-1(\bmod 3)$. By $(4.1) O^{r^{\prime}}\left(C_{G_{0}}^{0}(\tau a)\right)^{g} \leqslant C_{G_{0}}^{0}\left(E_{0}\right)$, so $C_{G_{0}}^{0}\left(E_{0}\right) \geqslant\left\langle L, O^{r^{\prime}}\left(C_{G_{0}}(\tau a)\right)^{g}\right\rangle$. Now let $I$ be the normal closure of $L$ in $C_{G_{0}}^{0}\left(E_{0}\right)$ and argue, using (2.8) (extended slightly to cover the case $E_{0} \$ G_{1}$ ), that $C_{G_{0}}^{0}\left(E_{0}\right)=G_{0}$. This requires a check that embeddings such as $\operatorname{PSL}(3, q) \leqslant P S L(4, q) \leqslant D_{4}(q)$ if $3 \mid q-1$ and $\operatorname{PS} U(3, q) \leqslant P S U(4, q) \leqslant D_{4}(q)$ if $3 \mid q+1$ are impossible. For this one can use representation theory (e.g. the tensor product theorem of Steinberg [24]).

We are left with the cases $q=2,4$. Here we construct an example $E_{0}$ for which (ii) holds. Let $a$ be an element of order 3 in $\left\langle U_{ \pm \alpha_{2}}\right\rangle$ and $x$ be as above. We may assume $\left\langle U: \alpha_{2}\right\rangle \leqslant L=C_{X}(\tau)$. Let $E_{00}=\langle x, \tau a, y\rangle$, where $y=y_{1} y_{2}$ with $y_{1} \in L$, $y_{2} \in Z-L,\left|y_{1}\right|=\left|y_{2}\right|=3, a^{y_{1}}=a x$, and $\tau^{y_{2}}=\tau x^{-1}$. Then $E_{00}$ is elementary abelian.

Let $P=O^{3}\left(C_{G_{0}}(\tau a)\right) \cong L_{3}(4)$ or $U_{3}(2)$. Now $\langle x\rangle=\left[E_{00},\langle a\rangle\right]$ and $a \in$ $C_{G_{0}}(\tau a)$, so $x \in P$. Also $C_{G_{0}}(a) \cap C_{G_{0}}(\tau) \geqslant S L_{2}(q)$ (as $C_{G_{0}}(\tau) \cong G_{2}(q)$ ). Thus $S L_{2}(q) \leqslant C_{G_{0}}(\tau a) \cap C_{G_{0}}(a)$, and $a$ induces outer automorphism on $P$. Let $\langle a, y\rangle \cap$ $P=\langle x\rangle \times\left\langle y^{1}\right\rangle$ for some $y^{1} \notin L$. Set $E_{0}=\left\langle x, y^{1}, \tau a\right\rangle$. We claim that $C_{G_{0}}^{0}\left(E_{0}\right) \leqslant$ $N_{\left(j_{0}\right.}\left(E_{0}\right)$.

Let $F$ be a hyperplane of $E_{0}$. If $F=\left\langle x, y^{1}\right\rangle$, then it is easy to see that $C_{G_{0}}(F) \leqslant N_{G_{0}}\left(E_{0}\right)$. Suppose $F \neq\left\langle x, y^{1}\right\rangle$. If $x \in F$, we again check that $C_{G_{0}}(F) \leqslant N_{G_{0}}\left(E_{0}\right)$. So suppose $x \notin F$. Then $F$ contains $\tau a x^{i}$ for some $i=0,1,2$. Now $\tau a x^{i} \sim \tau a$ by an element of $L$ normalizing $E_{0}$. Therefore, $E_{0} \cap G_{0} \leqslant O^{3}\left(C_{G_{0}}\left(\tau a x^{i}\right)\right)$ and we easily see that $C_{G_{0}}(F) \leqslant N_{G_{0}}\left(E_{0}\right)$. This proves the claim and completes the proof of (6.5).

We have now completed the proof of Theorem 2.

7. Additional results. In this section we will prove Theorems $3-7$. Theorem 3 is an immediate consequence of Theorems 1 and 2 so we concentrate on the notions of "layer generation" and "balance". Notation will be as in $\S 2$. Let $G_{1}$ be the group $G$ extended by all diagonal automorphisms of $G$. As in (2.1) we may prove Theorems 4 and 6 for the group $G_{0}$.

(7.1) Assume $Z_{p} \times Z_{p} \cong E<G_{1}$. Then one of the following holds:

(i) $C_{G_{0}}^{0}\left(E_{0}\right)<G_{0}$.

(ii) $C_{G_{0}}^{r}\left(E_{0}\right)=G_{0}$.

(iii) $p \mid q-1, G_{0} \cong S L(p, q)$, and $E_{0}$ is nonabelian.

(iv) $p \mid q+1, G_{0} \cong S U(p, q)$, and $E_{0}$ is nonabelian.

Proof. Suppose $C_{G_{0}}^{0}\left(E_{0}\right)=G_{0}$. Let $e \in E_{0}-Z\left(G_{0}\right)$ and let $\hat{D}_{1}, \ldots, \hat{D}_{k}$ be the products of the $\langle\sigma\rangle$-orbits of components of $C_{\hat{G}}(e)$. Let $T=Z\left(C_{\hat{G}}(e)\right)$ and for $i=1, \ldots, k$ set $L_{i}=O^{r^{\prime}}\left(\left(\hat{D}_{i}\right)_{\sigma}\right)$. Then $O^{r^{\prime}}\left(C_{G_{0}}(e)\right)=L_{1} \cdots L_{k}(\operatorname{see}(2.5))$.

First suppose that $E_{0}$ is abelian, so $E_{0} \leqslant C_{\hat{G}}(e)$ for each $e \in E_{0}-Z\left(G_{0}\right)$. By (2.4), $C_{\hat{G}}(e)=\hat{D}_{1} \cdots \hat{D}_{k} \hat{T}_{0}$. Choose $f \in E_{0}-\langle e\rangle Z\left(G_{0}\right)$ and consider $Y=C(f) \cap C_{\hat{G}}(e)$. If $Y_{i}^{0}=\left(Y \cap \hat{D}_{i}\right)^{0}$ is not a maximal torus of $\hat{D}_{i}$, then $\left(Y_{i}^{0}\right)_{\sigma}$ contains an element of order $r$, and (ii) follows from (2.3). So assume that $Y_{i}^{0}$ is a maximal torus of $\hat{D}_{i}$ for each $i=1, \ldots, k$. Then $Y^{0}$ is a $\sigma$-invariant maximal torus of $\hat{G}$ with $E_{0} \leqslant Y^{0}$. We conclude from (2.12) that $\left(C_{\hat{G}}(e)\right)_{\sigma}=L_{1} \cdots L_{k} Y_{\sigma}$ and from (2.11) that $C_{G_{0}}^{r}\left(E_{0}\right) \neq 1$. Then $\left(C_{\hat{G}}(e)\right)_{\sigma}=L_{1} \cdots L_{k} Y_{\sigma} \leqslant L_{1} \cdots L_{k} C_{G_{0}}\left(E_{0}\right) \leqslant N_{G_{0}}\left(C_{G_{0}}^{r}\left(E_{0}\right)\right)$. Since $e$ was arbitrary we have $G_{0}=C_{G_{0}}^{0}\left(E_{0}\right) \leqslant N\left(C_{G_{0}}^{r}\left(E_{0}\right)\right)$ and (ii) holds. 
Suppose $E_{0}$ is nonabelian. Then $p|| Z\left(G_{0}\right) \mid$ and $G \cong L_{n}(q), U_{n}(q), E_{6}(q)$, or ${ }^{2} E_{6}(q)$. In the first case $p \mid(n, q-1)$, in the second case $p \mid(n, q+1)$, and in the other cases $p=3$. If $G=L_{n}(q)$, then as in $\S 4$ we may assume $E_{0}$ acts on the usual module for $G_{0}=S L(n, q)$. If $E_{0}$ acts reducibly, then $E_{0}$ is contained in a parabolic subgroup of $G_{0} E_{0}$ and (ii) follows from (2.3). If $E_{0}$ acts irreducibly, then elementary arguments from representation theory yield $n=p$, and (ii) holds.

Next, suppose $G_{0}=S U(n, q)$. Then we may assume that $E_{0}$ is a subgroup of

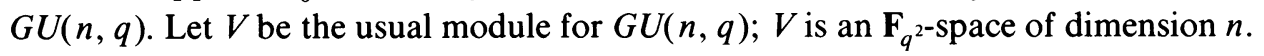
Let $e \in E_{0}-Z\left(G_{0}\right)$ with $|e|=p$, and let $f \in E_{0}-\langle e\rangle Z\left(G_{0}\right)$. If $\langle z\rangle=E_{0} \cap Z\left(G_{0}\right)$, then we may assume $e^{f}=e z$. The eigenspaces of $e$ on $V$ are permuted transitively by $\langle f\rangle$ and each has dimension $n / p$. Let $V_{1}, \ldots, V_{p}$ be these eigenspaces. Using the fact that 1 is an eigenvalue of $e$, an easy check gives $V=V_{1} \perp \cdots \perp V_{p} . \operatorname{If} \operatorname{dim}(V)=p$, then (iv) holds. So suppose $\operatorname{dim}(V)>p$; that is, $\operatorname{dim}\left(V_{i}\right)>1$ for $i=1, \ldots, p$. Let $0 \neq v_{1} \in V_{1}$ be an isotropic vector and $\left\langle v_{1}\right\rangle, \ldots,\left\langle v_{p}\right\rangle$ the images of $\left\langle v_{1}\right\rangle$ under $\langle f\rangle$. Then $\left\langle v_{1}, \ldots, v_{p}\right\rangle=\left\langle v_{1}\right\rangle \perp \cdots \perp\left\langle v_{p}\right\rangle$ is an $E_{0}$-invariant isotropic subspace of $V$. Therefore $E_{0}$ is contained in a proper parabolic subgroup of $G U(n, q)$ and (ii) follows from (2.3).

Finally, we assume that either $G_{0}=E_{6}(q)$ with $p=3 \mid q-1$ or $G_{0}={ }^{2} E_{6}(q)$ and $p=3 \mid q+1$. Let $e, f \in E_{0}$ with $[e, f] \neq 1$. Then $f \notin C_{\hat{G}}(e)$, although $f \in N\left(C_{\hat{G}}(e)\right)$. This is because $C_{\hat{G}}(e)=C_{\hat{G}}\left(e^{f}\right)$. By (2.3) we may assume that $f$ normalizes no proper $r$-subgroup of $C_{G_{0}}(e)$. Then considering the $\langle f\rangle$-orbits on components in $C_{\hat{G}}(e)$ and then the action of $\sigma$ on these orbits we see that $f$ must normalize each component of $C_{\hat{G}}(e)$. Also, if we choose $e$ such that $|e|=3$, then it is easy to see that $E\left(C_{\hat{G}}(e)\right) \neq 1$. Indeed, just embed $e$ in a maximal torus of $\hat{G}$ and consider the action of $e$ on the unipotent radical of a Borel group. Let $Y$ be a component of $C_{\hat{G}}(e)$. Then $Y=Y^{f}$ and there is a root subgroup, $V \leqslant Y$, with $V=V^{f}$. To see this just consider the isomorphism that $f$ induces on $Y$. By (7.5) of Steinberg [23], $f$ stabilizes a Borel subgroup of $Y$, and we let $V$ be the center of the unipotent radical of the Borel subgroup.

Now consider $E_{0}$ as a subgroup of $\hat{P}=N_{\hat{G}}(V) . \hat{P}$ is a parabolic subgroup of $\hat{G}$ with $\hat{P} / \hat{P}_{u} \cong S L(6, K) I$, where $I=I^{0}$ is a one-dimensional torus centralizing the $S L(6, K)$ factor. By (5.16) of [22], $E_{0}$ stabilizes a maximal torus of $\hat{P}$ and we may assume $E_{0} \leqslant N_{\hat{P}}(\hat{I})$, where $\hat{I}$ is a torus of $\hat{G}$ with $\hat{I} \hat{P}_{u} / \hat{P}_{u}=I$. So $E_{0}$ is in a Levi factor of $\hat{P}$ and it follows that $C_{\hat{P}}\left(E_{0}\right)$ contains a component. But $C(f) \cap C_{\hat{G}}(e)$ does not contain a component. This is impossible. This completes the proof of (7.1).

Theorem 4 (LAYer Generation). Let $Z_{p} \times Z_{p} \cong E \leqslant \operatorname{Aut}(G)$. Then one of the following holds:

(i) $C_{G_{0}}^{0}\left(E_{0}\right)<G_{0}$.

(ii) $C_{G_{0}}^{r}\left(E_{0}\right)=G_{0}$.

(iii) $p \mid q-1, G_{0} \cong S L(p, q), E \leqslant G_{1}$, and $E_{0}$ is nonabelian.

(iv) $p \mid q+1, G_{0} \cong S U(p, q), E \leqslant G_{1}$, and $E_{0}$ is nonabelian.

Proof. By (7.1) we may assume $E \$ G_{1}$, and we may also assume that $C_{G_{0}}^{0}\left(E_{0}\right)=G_{0}$. First suppose that it is not the case that $p=3$ and $G \cong D_{4}(q)$ or 
${ }^{3} D_{4}(q)$. Then Lang's theorem [18] implies that $E-\left(E \cap G_{1}\right)$ consists of field automorphisms of $G$. As in the proof of (6.1) we use Theorem 1 of [8] to get $C_{G_{0}}^{r}\left(E_{0}\right)=O^{r^{\prime}}\left(C_{G_{0}}(e)\right)$ for each $e \in E_{0}$ with $e Z\left(G_{0}\right) \notin G_{1}$. But then $E_{0}$ normalizes a proper parabolic subgroup of $G_{0}$, and (2.3) gives (ii).

Suppose $p=3$ and $G \cong D_{4}(q)$ or ${ }^{3} D_{4}(q)$. Either $E_{0} \cap G_{0} \cong Z_{3}$ or $G \cong D_{4}(q)$ and $E \cap G_{1}=1$. In the latter case the argument of the first paragraph of the proof of (6.4) gives $C_{G_{0}}^{r}\left(E_{0}\right)=G_{0}$. So suppose $E_{0} \cap G_{0}=\langle e\rangle \cong Z_{3}$. In addition we may assume that $G_{0} E_{0}=G_{0}\langle\tau\rangle$, for $\tau$ a graph automorphism of $G_{0}$. For otherwise use the argument of the above paragraph.

Suppose $f \in E_{0}-\langle e\rangle$ and $f$ induces a graph automorphism on $G_{0}$. Then $C_{G_{0}}(f) \cong G_{2}(q)$ and we consider $C_{G_{0}}(f) \leqslant G_{2}(K)=Y$, where $Y=\hat{G}_{f}$. Now embed $e$ in a maximal torus of $Y$ and then consider the action of $e$ on the unipotent radical of a Borel subgroup of $Y$. It is easy to deduce that $E\left(C_{Y}(e)\right) \neq 1$, so applying $\sigma$ to this group and using (2.3), we again see that (ii) holds. From now on we assume that $E_{0}$ contains no conjugate of $\tau$.

Suppose $E_{0} \leqslant C_{G_{0}}(\tau) \times\langle\tau\rangle \cong G_{2}(q) \times Z_{3}$. $C_{G}(\tau)$ contains a subgroup, $L$, such that $L \cong S L(3, q)$ or $S U(3, q)$ depending on whether $q \equiv 1,-1(\bmod 3)$. By order considerations we see that $L$ contains a Sylow 3-subgroup of $C_{G}(\tau)$ so we take $E_{0} \leqslant L \times\langle\tau\rangle$. As $\tau \notin E_{0}$, the projection of $E_{0}$ to $L$ contains a representative of each class of elements of order 3 in $L$. However, it is easy to check that $\tau \sim \tau l$ for some element, $l$, of order 3 in $L$. This contradicts the above.

Suppose $G_{0} \cong{ }^{3} D_{4}(q)$ or $G_{0} \cong D_{4}(q)$ with $q>4$. Then the argument in the fourth paragraph of the proof of (6.5) shows that $G_{0}=C_{G_{0}}^{r}\left(E_{0}\right)$, proving (ii). So now assume $G_{0}=D_{4}(q)$ with $q=2$ or 4 .

We use the notation in the proof of (6.5). Write $E_{0}=\langle e\rangle \times\langle\tau a\rangle$, where $C_{G_{0}}(\tau a) \cong P G U(3,2)$ or $P G L(3,4)$, depending on whether $q=2$ or 4 . If $e \in O^{2}\left(C_{G_{0}}(\tau a)\right)$, then $e$ is 3-central in $C_{G_{0}}(\tau a)$. Since $\tau \in C_{G_{0}\langle\tau\rangle}(\tau a)$, this would imply that $E_{0}$ centralizes a conjugate of $\tau$, which we have just seen to be impossible. Therefore $e \notin O^{2}\left(C_{G_{0}}(\tau a)\right)$ and $C_{G_{0}}(\tau a)=O^{2^{\prime}}\left(C_{G_{0}}(\tau a)\right)\langle e\rangle$. Each $f \in E_{0}-\langle e\rangle$ satisfies $f \sim \tau a$, so $C_{G_{0}}(f)=O^{2}\left(C_{G_{0}}(f)\right)\langle e\rangle$, for each $f \in E_{0}-\langle e\rangle$.

If we also have $C_{G_{0}}(e)=O^{2}\left(C_{G_{0}}(e)\right)\langle e\rangle$, then $C_{G_{0}}^{0}\left(E_{0}\right) \leqslant N_{G_{0}}\left(C_{G_{0}}^{r}\left(E_{0}\right)\right)=X$, and the result follows. So suppose $C_{G_{0}}(e) \$ X$. Then $C_{G_{0}}(e)>O^{2^{\prime}}\left(C_{G_{0}}(e)\right)\langle e\rangle$ and by [7] we have $e \sim x$, where $x$ is as in the proof of (6.5). Consider $C_{G_{0}}(e)$. This group can be expressed $C_{G_{0}}(e)=Z\langle u, d\rangle$, where $Z=O^{2^{\prime}}\left(C_{G_{0}}(e)\right) \cong S U(3,2)$ or $S L(3,4)$, depending on whether $q=2$ or $4, Z\langle u\rangle \cong G U(3,2)$ or $G L(3,4),|d|=3$, and $C_{G_{0}}(e)=Z\langle u\rangle \times\langle d\rangle$. Now $\tau a$ acts on $C_{G_{0}}(e)$ and $[\tau a, d]=e^{ \pm 1}$. So $d \in N\left(E_{0}\right) \leqslant X$ and a Sylow 3-subgroup of $X$ has index at most 3 in a Sylow 3-subgroup of $G_{0}$.

We claim that $X$ contains a Sylow 3-subgroup of $G_{0}$. Suppose false. If $S \in \operatorname{Syl}_{3}\left(C_{X}(e)\right)$ with $S^{\tau a}=S$, then $S \in \operatorname{Syl}_{3}(X), S=\left\langle x_{1}, x_{2}\right\rangle \times\langle d\rangle$, where $\left\langle x_{1}, x_{2}\right\rangle=S \cap Z$. Let $V$ be the natural module for $G_{0}$. We may assume $\operatorname{dim}\left(C_{V}(d)\right)=6$ and $Z$ acts irreducibly on $C_{V}(d)$, while centralizing $[V, d]$. It follows that $e$ is fixed-point-free on $C_{V}(d)$, but elements of $\left\langle x_{1}, x_{2}\right\rangle-\langle e\rangle$ centralize a 2-space of $C_{V}(d)$. Now consider $C_{G_{0}}(\tau a) \cong P G U(3,2)$ or $P G L(3,4)$. We have seen that $e \notin C_{G_{0}}(\tau a)^{\prime}$ and $\operatorname{dim}\left(C_{V}(e)\right)=2$. Also $C_{G_{0}}(\tau a)^{\prime}$ acts irreducibly on $V$ and it is 
easy to see that each element of order 3 in $C_{G_{0}}(\tau a)^{\prime}$ centralizes just a 2-space in $V$. So $C_{G_{0}}(\tau a)$ contains a subgroup of order 27 of the form $\langle e, j\rangle$, with $\langle e, j\rangle$ extraspecial. Apply Sylow's theorem to $X\langle\tau a\rangle$ and conclude that $\left(C_{G_{0}}(e)^{\prime} \times\langle d\rangle\right)\langle\tau a\rangle$ contains a conjugate of $\langle e, j\rangle \times\langle\tau a\rangle$. This forces $\langle e, j\rangle \sim\left\langle x_{1}, x_{2}\right\rangle$ in $G_{0} . \operatorname{But} \operatorname{dim}\left(C_{V}(e)\right)=2$, while $\operatorname{dim}\left(C_{V}(w)\right)=4$ for each $w \in\left\langle x_{1}, x_{2}\right\rangle-\langle e\rangle$. This is a contradiction. Thus $X$ contains a Sylow 3-subgroup of $G_{0}$. Let $S<\bar{S} \in \operatorname{Syl}_{3}(X)$. Then $|\bar{S}: S|=3$ and from the above considerations we see that $\langle e\rangle$ is weakly closed in $Z(S)$. So $\bar{S} \leqslant C_{G_{0}}(e)$ and we now have $C_{G_{0}}(e) \leqslant X$. This contradicts the earlier assumption. We have now proved Theorem 4.

Next, we will consider the notion of balance.

(7.2) Suppose $Z_{p} \times Z_{p} \cong E \leqslant G_{1}$. Then one of the following holds:

(i) $C_{G}^{0}(E)<G$.

(ii) $\cap{ }_{e} O_{p^{\prime}}\left(C_{X}(e)\right)=1$ for any $G \leqslant X \leqslant \operatorname{Aut}(G)$, the intersection ranging over $e \in E^{\#}$.

(iii) $G \cong L_{p}(q), p \mid q-1$, and $E_{0}$ is nonabelian.

(iv) $G \cong U_{p}(q), p \mid q+1$, and $E_{0}$ is nonabelian.

Proof. Suppose that (i), (iii), and (iv) are false. Then Theorem 4 and (2.1) imply that $C_{G}^{r}(E)=G$. So it will suffice to prove that if $1 \neq g \in O_{p^{\prime}}\left(C_{X}(e)\right)$ for each $e \in E^{\#}$ and some $G \leqslant X \leqslant \operatorname{Aut}(G)$, then $C_{G}^{r}(E) \leqslant C_{G}(g)$. Fix such an element $g$ with $g$ of prime order, and $e \in E^{\#}$.

Choose a $p$-element $e_{1} \in \hat{G}$ such that $e_{1}$ projects to the element $e \in \bar{G}$. Then $Y=C_{\hat{G}}\left(e_{1}\right)=E_{1} \cdots E_{k} T$, where the product is a commuting product of the connected torus $T$ and the components $E_{1}, \ldots, E_{k}$ (see (2.5)). Passing to $\bar{G}$, let

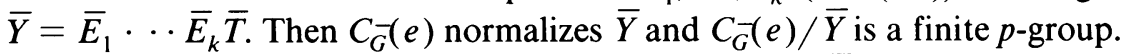

Let $\bar{D}_{1}, \ldots, \overline{D_{l}}$ be the orbit products of $\sigma$ on $\left\{\bar{E}_{1}, \ldots, \bar{E}_{k}\right\}$ and set $L_{i}=O^{r^{\prime}}\left(\left(\bar{D}_{i}\right)_{\sigma}\right)$. Then $O^{r^{\prime}}\left(C_{G}(e)\right)=L_{1} \cdots L_{l}$. The element $g$ permutes the components (solvable components) $L_{1}, \ldots, L_{l}$ of $C_{G}(e)$. Suppose $\left[L_{i}, g\right] \neq 1$ for some $i=1, \ldots, l$. Since $g \in O_{p^{\prime}}\left(C_{X}(e)\right)$, we have $\left[L_{i}, g\right]$ a $p^{\prime}$-group. It follows that $L_{i}$ is a $p^{\prime}$-group. This is clear if $L_{i}^{g} \neq L_{i}$. If $L_{i}^{g}=L_{i}$, then $\left[L_{i}, g\right]=L_{i}$ unless $L_{i}$ is a solvable component, and in this case the assumption $p \neq 2, r$ gives the assertion. In particular, $p \nmid q^{2}-1$ unless $L_{i}$ is a Suzuki group.

The condition $p \nmid q^{2}-1$ implies that $Z\left(G_{0}\right)$ has order prime to $p$ and $E \cong E_{0} \cong Z_{p} \times Z_{p}$. So $E_{0} \leqslant C_{\hat{G}}\left(e_{1}\right)$ and it follows that $\left[L_{i}, E_{0}\right] \leqslant L_{i}$. Also, if $f \in E_{0}-\left\langle e_{1}\right\rangle$, then $f$ induces the product of an inner and diagonal automorphism on $L_{i}$. However, $L_{i}$ is a $p^{\prime}$-group and $p \nmid q^{2}-1$. It follows that $\left[L_{i}, f\right]=\left[L_{i}, E_{0}\right]=1$. If $L_{i}$ is a Suzuki group, then $q$ is even and $\bar{G} \cong S p(2 n, K)$ or $F_{4}(K)$. Thus $Z(\hat{G})=1$ and we can use the same argument to obtain $\left[L_{i}, E_{0}\right]=1$.

Choose $f \in E^{\#}$ and consider $L_{i} \leqslant O^{r^{\prime}}\left(C_{G}(f)\right)$. $f$ acts on each component of $C_{G}(e)$ and we claim that $\left[f, \bar{D}_{i}\right]=1$. Suppose $\bar{D}_{i}$ is the product of $n$ components of $C_{G}(e)$. Then $\left\langle\sigma^{n}\right\rangle$ is the stabilizer of each of these components and we take a component $I \leqslant \bar{D}_{i}$. Then $I^{\sigma^{n}}=I$ and $\left[O^{r^{\prime}}\left(I_{\sigma^{n}}\right), f\right]=1$. From (2.1) of [8] we conclude that $[I, f]=1$. The claim follows.

Since $E_{0}$ is abelian we choose $f \in E_{0}-\left\langle e_{1}\right\rangle$ and embed $f$ in a maximal torus, $\hat{H}$, of $C_{\hat{G}}\left(e_{1}\right)=C_{\hat{G}}\left(e_{1}\right)^{0}$. Then $E_{0}<\hat{H}$ and we let $\bar{H} \leqslant \bar{B}$, where $E \leqslant \bar{H}$ is a maximal 
torus of $\bar{G}$ and $\bar{B}$ is a Borel subgroup of $\bar{G}$. By (4.1) of [22] the components of $C_{\bar{G}}(f)$ are generated by the root subgroups in $\bar{G}_{u}$ that are centralized by $f$. It follows that the normal closure of $\bar{D}_{i}$ in $C_{\bar{G}}(f)^{0}$, call it $\bar{K}_{i}$, is the product of a $\langle\sigma\rangle$-orbit of components of $C_{\bar{G}}(f)$. Set $X_{i}=O^{r^{\prime}}\left(\left(\bar{K}_{i}\right)_{\sigma}\right)$. Then $\left[\bar{L}_{i}, g\right] \neq 1$ implies $\left[X_{i}, g\right] \neq 1$.

Now rechoose the pair $\left(L_{i}, e\right)$, if necessary, so that $\left|L_{i}\right|$ is maximal subject to the conditions that $L_{i}$ is a component (solvable component) of $C_{G}(e)$ with $\left[L_{i}, g\right] \neq 1$. We then have $L_{i}=X_{i} \leqslant O^{r^{\prime}}\left(C_{G}(f)\right)$. So $N_{G}\left(L_{i}\right) \geqslant C_{G}^{r}(e)=G$, a contradiction. This proves that $\left[g, L_{i}\right]=1$ for each component (solvable component), $L_{i}$, of $C_{G}(e)$ and for each $e \in E^{\#}$. Therefore $G=C_{G}^{r}(E) \leqslant C_{G}(g)$ which is impossible.

The next result handles case (i) of (7.2).

(7.3) Suppose $Z_{p} \times Z_{p} \cong E \leqslant G_{1}$ and $C_{G}^{0}(E)<G$. Then one of the following occurs:

(i) $\cap_{1 \neq e} O_{p^{\prime}}\left(C_{X}(e)\right)=1$, for any $G \leqslant X \leqslant \operatorname{Aut}(G)$.

(ii) $p=3$ and $G=L_{3}(4)$.

Proof. Apply Theorem 1 to restrict the possibilities for $G$ and $p$. In each case $\left|G_{1}: G\right|=1$ or $p$. Let $Y=\bigcap_{1 \neq e} O_{p^{\prime}}\left(C_{G_{1}}(e)\right)$. We will first show that $Y=1$.

If $G={ }^{2} F_{4}(2)^{\prime}$ with $p=5$, then $E \in \operatorname{Syl}_{5}(G)$ and there is an element $e \in E^{\#}$ with $C_{G}(e) \cong Z_{5} \times D_{10}$. This already implies $Y=1$. So we now assume $p=3$ and $q=2$ or 4. We may then apply the results of [7]. There are only two possible configurations where some element $e \in E^{\#}$ satisfies $O_{3^{\prime}}\left(C_{G}(e)\right) \neq 1$. The cases are $G \cong P S L\left(3^{k}, 4\right)$ and $G=E_{6}(4)$, and $O_{3^{\prime}}\left(C_{G}(e)\right) \cong Z_{7}$ in either case.

So supposing $Y \neq 1$ we must have $Y=Z_{7}$ and $O_{3^{\prime}}\left(C_{G}(e)\right)=O_{3^{\prime}}\left(C_{G}(f)\right)$ for each $f \in E^{\#}$. Also $C_{G}^{0}(E) \leqslant N_{G}(Y)$, so by (2.3), $E$ is contained in no proper parabolic subgroup of $G$. For $G=\operatorname{PSL}\left(3^{k}, 4\right)$ this implies that the preimage of $E$ in $G L\left(3^{k}, 4\right)$ acts irreducibly on the usual module for $G L\left(3^{k}, 4\right)$. Then $E \cong Z_{3} \times Z_{3}$ implies $k=1$, and it is easy to see that $Y=1$.

Suppose $G=E_{6}(4)$. By [7] we have $E\left(C_{G}(e)\right) \cong{ }^{3} D_{4}(4)$ for each $e \in E^{\#}$. Fix $e \in E^{\#}$ and $f \in E-\langle e\rangle$. Then use [7] to see that $C(f) \cap E\left(C_{G}(e)\right)$ has even order. So $E$ is contained in a parabolic subgroup of $G_{1}$ and (2.3) implies that $C_{G}^{0}(E)=G$, against our hypothesis. Therefore $Y=1$.

Set $Y_{1}=\bigcap_{1 \neq e} O_{p^{\prime}}\left(C_{\mathrm{Aut}(G)}(e)\right)$ and suppose $Y_{1} \neq 1$. By the above $Y_{1} \cap G_{1}=1$. Considering the possibilities for $G$ we see that $Y_{1}$ is quite restricted. For example if $G={ }^{2} F_{4}(2)^{\prime}$, then $\operatorname{Aut}(G)={ }^{2} F_{4}(2)$ and there are no involutions in $\operatorname{Aut}(G)-G$. So this case is out. In the remaining cases $\operatorname{Aut}(G) / G_{1} \cong 1, Z_{2}, Z_{2} \times Z_{2}, Z_{4}$, or $S_{3}$ (the latter possible only if $\left.G=D_{4}(2)\right)$. So $Y_{1} \cong Z_{2}, Z_{4}$, or $Z_{2} \times Z_{2}$. Let $y$ be an involution in $Y_{1}$.

The possible actions of $y$ on $G$ are determined in $\$ 19$ of [3]. Namely, $y$ induces a field automorphism, graph automorphism, graph-field automorphism, or $O_{2}\left(C_{G}(y)\right) \neq 1$. This last case contradicts (2.3), so this does not occur. Choose $e \in E^{\#}$ and let $\bar{L}_{1}, \ldots, \bar{L}_{l}$ be the components (solvable components) of $C_{G}(e)$. By (2.3) $И(r, E)=\{1\}$, and it follows that 3||$L_{i} \mid$ for $i=1, \ldots, l$. However $\left[\bar{L}_{i}, y\right]$ is a $3^{\prime}$-group for each $i=1, \ldots, l$. Since $r=2$ we necessarily have $\left[\bar{L}_{i}, y\right]=1$ for $i=1, \ldots, l$. The conclusion is that $C_{G}^{2}(E)=C_{C_{G}(y)}^{2}(E)$.

If $G=P S L\left(3^{k}, 4\right)$, then as before we get $k=1$. So (ii) holds. We assume now that $G \neq P S L\left(3^{k}, 4\right)$. If $G=P S U(n, 2)$, then $E \leqslant C_{G}(y)=\operatorname{Sp}(n, 2)$ or 
$S p(n-1,2)$, so the preimage of $E$ in $G U(n, 2)$ is elementary abelian. Here (4.1) and linear algebra show that the condition $C_{G}^{2}(E)=C_{C_{G}(y)}^{2}(E)$ is impossible, Similarly for $G=O^{ \pm}(n, 2)^{\prime}$.

For the remaining cases use the tables in [7] to check that $C_{G}^{2}(E) \neq C_{C_{G}(y)}^{2}(E)$.

We now prove

Theorem 5 (BALANCE). If $Z_{p} \times Z_{p} \cong E \leqslant \operatorname{Aut}(G)$, then $\bigcap_{e \neq 1} O_{p^{\prime}}\left(C_{X}(e)\right)=1$ for any $G \leqslant X \leqslant G_{1}$ and one of the following holds:

(i) $\cap \cap_{e \neq 1} O_{p^{\prime}}\left(C_{X}(e)\right)=1$, for any $G \leqslant X \leqslant \operatorname{Aut}(G)$.

(ii) $p \mid q-1, G \cong L_{p}(q), E \leqslant G_{1}$ and $E_{0}$ is nonabelian.

(iii) $p \mid q+1, G \cong U_{p}(q), E \leqslant G_{1}$, and $E_{0}$ is nonabelian.

Proof. Suppose $1 \neq g \in \bigcap_{1 \neq e} O_{p^{\prime}}\left(C_{\mathrm{Aut}(G)}(e)\right)$. First assume that either (7.2)(iii) or (7.2)(iv) holds. Then $E_{0}$ is nonabelian and is absolutely irreducible on the usual module for $G_{0}$. It follows that $g \notin G_{1}$. This together with (7.2) and (7.3) allow us now to assume $E \$ G_{1}$. Suppose each $e \in E-G_{1}$ induces a field automorphism on $G$. For $e \in E-G_{1}, p$ divides the order of $C_{G}(e)$ and it follows that $p$ divides the order of $O^{r^{\prime}}\left(C_{G}(e)\right)$. So $g \in \bigcap_{1 \neq e} O_{p^{\prime}}\left(C_{\mathrm{Aut}(G)}(e)\right)$ implies $\left[O^{r^{\prime}}\left(C_{G}(e)\right), g\right]=1$. As in the proof of (6.1) we apply Theorems 1 and 2 of [8]. It is necessary to look at the cases $G \cong L_{2}\left(2^{p}\right), L_{2}\left(3^{p}\right)$, and $S z\left(2^{p}\right)$ individually, but this is straightforward. For all the other cases we have $O^{r^{\prime}}\left(C_{G}(e)\right) \leqslant C_{G}(g)$ for each $e \in E-G_{1}$ and so $O^{r^{\prime}}\left(C_{G}(e)\right)=O^{r^{\prime}}\left(C_{G}(g)\right)$ for each $e \in E-G_{1}$. So $E \leqslant C\left(O^{r^{\prime}}\left(C_{G}(g)\right)\right)$ and looking at $E \cap G_{1}$, we contradict (2.1) of [8].

So we now suppose that some $e \in E-G_{1}$ does not induce a field automorphism on $G$. Then $p=3$, and $G \cong D_{4}(q)$ or ${ }^{3} D_{4}(q)$. The above arguments allow us to assume that $E \leqslant G_{1}\langle\tau\rangle$, where $\tau$ is a graph automorphism of $G$ with $|\tau|=3$. As in the fourth paragraph of the proof of (6.5) we argue that for $e \in E-G_{1}$, $C_{G}(e) \cong G_{2}(q), P G L(3, q)$, or $P G U(3, q)$. The first case occurs if $e$ is conjugate to $\tau$, and one of the other cases (according to $q \equiv 1,-1(\bmod 3))$ otherwise. For $q>2$ and $e \in E \cap G_{1}, O^{r^{\prime}}\left(C_{G}(e)\right)=E\left(C_{G}(e)\right)$ (see (2.5)). So under the assumption $q>2$ we argue as before (e.g. as in (7.2)) that $\left[O^{r^{\prime}}\left(C_{G}(e)\right), g\right]=1$ for each $e \in E^{\#}$. Then $C_{G}^{r}(E) \leqslant C_{G}(g)$ and we have a contradiction if $C_{G}^{r}(E)=G$. By Theorems 1, 2, and 4 we are reduced to the cases $G=D_{4}(2), D_{4}(4)$, or ${ }^{3} D_{4}(2)$.

Choose $e \in E-G_{1}$. The possible choices (listed above) for $C_{G}(e)$ and the results of [7] show that $g \notin G_{1}$. Considering $\operatorname{Aut}(G)$ we see that $|g|=2, G=D_{4}(4)$, and $g$ induces a field automorphism on $G$. Then $\left[g, C_{G}(e)\right]=C_{G}(e)^{\prime}\left(=G_{2}(4)\right.$ or $\left.L_{3}(4)\right)$, a contradiction. This completes the proof of Theorem 5.

THEOREM 6. Suppose $p \geqslant 7$ and $(p,|\bar{W}|)=1$, where $\bar{W}$ is the Weyl group of the associated algebraic group of $G$. Then $C_{G_{0}}^{r}\left(E_{0}\right)=G_{0}$.

Proof. First suppose $E \leqslant G_{1}$. Then (2.7) implies that $E_{0}$ is contained in a maximal torus of $\hat{G}$ and $C_{\hat{G}}(e)=C_{\hat{G}}(e)^{0}$ for each $e \in E_{0}^{\#}$. Choose $Z_{p} \times Z_{p} \cong F \leqslant E_{0}$. By Theorems 4 and 1 we have $C_{G_{0}}^{r}(F)=G_{0}$. For $f \in F^{\#}$ each component (solvable component) of $O^{r^{\prime}}\left(C_{G_{0}}(f)\right)$ has Weyl group with order dividing that of $|\bar{W}|$. This is because such a component is obtained as the fixed point group of $\sigma$ on a product of components of $C_{\hat{G}}(f)$ and each of the components of $C_{\hat{G}}(f)$ is 
generated by the root groups in a subsystem of $\hat{\Sigma}$ (see (4.1) of [22]). So we can apply induction to get $O^{r^{\prime}}\left(C_{G_{0}}(f)\right) \leqslant C_{G_{0}}^{r}\left(E_{0}\right)$. Then $G_{0}=C_{G_{0}}^{r}(F) \leqslant C_{G_{0}}^{r}\left(E_{0}\right)$.

Suppose $E \$ G_{1}$. Then $E-\left(E \cap G_{1}\right)$ consists of field automorphisms and the arguments of (6.1) give the result.

Our last result determines the strongly $p$-embedded subgroups of Chevalley groups. For this we drop the assumption $p \neq 2, r$.

THEOREM 7. Let $p$ be an arbitrary prime and suppose $X \leqslant \operatorname{Aut}(G)$ is a proper, strongly p-embedded subgroup of $G X$. Assume $m_{p}(X) \geqslant 2$. Then $X$ is contained in the normalizer of a Sylow p-subgroup of $G$ and one of the following holds:

(i) $p=3$ and $G \cong L_{3}(4)$.

(ii) $p=5$ and $G \cong S z\left(2^{5}\right) o r^{2} F_{4}(2)^{\prime}$.

(iii) $G \cong L_{2}(q), U_{3}(q), S z(q)$, or ${ }^{2} G_{2}(q)^{\prime}$, where $q$ is a power of $p$.

Proof. Assume $X$ is strongly $p$-embedded in $G X$. Then $X$ contains a Sylow $p$-subgroup, $P$, of $G$. If $p=2$, apply Bender [4] to get the result. If $p=r$, then either $G$ has Lie rank 1 (which gives (iii)) or $G$ is generated by the proper parabolic subgroups of $G$ that contain $P$. As these groups are $p$-local subgroups, we have $G \leqslant H$, a contradiction. So we may now assume $p \neq 2, r$.

For any $Z_{p} \times Z_{p} \cong E \leqslant X$ we have $C_{G}^{0}(E) \leqslant X$. So Theorems 1 and 2 restrict the possibilities for $G$. If $p=5$, then (i) holds. So now assume $p=3$ and $q$ is a power of 2. If $G$ has Lie rank at least 3, then from the Dynkin diagram it is easy to produce a proper parabolic subgroup of $G$ containing two commuting copies of $L_{2}(q)$. But then there exists $Z_{3} \times Z_{3} \cong E \leqslant X \cap G$ with $E$ in a proper parabolic subgroup of $G$ and $C_{G}^{0}(E)<G$. This contradicts (2.3). Therefore $G$ has Lie rank at most 2.

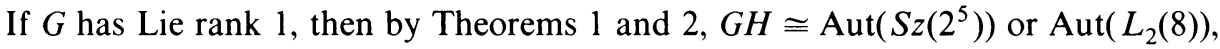
so that (ii) or (iii) holds. Suppose $G$ has Lie rank 2 . Then $m_{3}(G) \geqslant 2$ and there exists $Z_{3} \times Z_{3} \cong E \leqslant X \cap G$, satisfying $C_{G}^{0}(E)<G$. Theorem 1 now gives $G \cong L_{3}(4)$, $P S p(4,2)^{\prime}, P S U(5,2), G_{2}(2)^{\prime}$, or ${ }^{2} F_{4}(2)^{\prime}$. If $G=G_{2}(2)^{\prime}$, then $G \cong U_{3}(3)$ and (iii) holds. If $G \cong{ }^{2} F_{4}(2)^{\prime}$ with $X<G$, then $X \cong \operatorname{Aut}\left(L_{3}(3)\right)$ and $X=\left\langle N_{G}(E)\right.$ : $\left.Z_{3} \times Z_{3} \cong E \leqslant P\right\rangle$. On the other hand, choosing $g \in{ }^{2} F_{4}(2)-{ }^{2} F_{4}(2)^{\prime}$ with $g \in N(P)$, we must have $X^{g}=X$. But then $C_{G_{1}}(X)$ contains an involution in $G_{1}-G$, whereas $G_{1}-G$ contains no involution.

If $G \cong P S U(5,2)$, a Borel subgroup of $G$ has 3-rank 2. So this case is out by (2.3). Thus $G \cong L_{3}(4)$ or $P S p(4,2)^{\prime} \cong L_{2}(9)$, and Theorem 7 is proved.

\section{REFERENCES}

1. J. Alperin and R. Lyons, On the conjugacy classes of p-elements, J. Algebra 19 (1971), 536-537.

2. M. Aschbacher, A characterization of Chevalley groups over fields of odd order. I, II, Ann. of Math. 106 (1977), 353-398, 399-468.

3. M. Aschbacher and G. Seitz, Involutions in Chevalley groups over fields of even order, Nagoya Math. J. 63 (1976), 1-91.

4. H. Bender, Transitive Gruppen gerader Ordnung, in denen jede Involution genau einen Punkt festlässt, J. Algebra 17 (1971), 527-554.

5. D. Bloom, The subgroups of PSL $(3, q)$ for odd $q$, Trans. Amer. Math. Soc. 127 (1967), 150-178.

6. A. Borel and J. Tits, Eléments unipotents et sousgroupes paraboliques de groupes reductifs. I, Invent. Math. 12 (1971), 95-104.

7. N. Burgoyne, Elements of order 3 in Chevalley groups of characteristic 2 (to appear). 
8. N. Burgoyne, R. Griess and R. Lyons, Maximal subgroups and automorphisms of Chevalley type groups, Pacific J. Math. 71 (1977), 365-403.

9. N. Burgoyne and C. Williamson, Semi-simple classes in Chevalley type groups (to appear).

10. C. Curtis, W. Kantor and G. Seitz, The two transitive permutation representations of the finite Chevalley groups, Trans. Amer. Math. Soc. 218 (1976), 1-59.

11. B. Fischer, Finite groups generated by 3-transpositions, Invent. Math. 13 (1971), 232-246.

12. P. Fong and G. Seitz, Groups with a (B, N)-pair of rank 2. II, Invent. Math. 24 (1974), 191-239.

13. D. Goldschmidt, 2-fusion in finite groups, Ann. of Math. (2) 99 (1974), 70-117.

14. R. W. Hartley, Determination of the ternary collineation groups whose coefficients lie in the $G F\left(2^{n}\right)$, Ann. of Math. (2) 27 (1926), 140-158.

15. D. Higman and J. McLaughlin, Geometric ABA-groups, Illinois J. Math. 5 (1961), 382-397.

16. A. Kurosh, The theory of groups, vol. 2, Chelsea, New York, 1960.

17. V. Landazuri and G. Seitz, On the minimal degrees of projective representations of the finite Chevalley groups, J. Algebra 32 (1974), 418-443.

18. S. Lang, Algebraic groups over finite fields, Amer. J. Math. 18 (1956), 555-563.

19. J. McLaughlin, Some groups generated by transvections, Arch. Math. 18 (1967), $364-368$.

20. B. Mwene, On the subgroups of the group $P S L_{4}\left(2^{m}\right)$, J. Algebra 41 (1976), 79-107.

21. G. Seitz, Flag transitive subgroups of Chevalley groups, Ann. of Math. (2) 97 (1973), 27-56.

22. T. Springer and R. Steinberg, Conjugacy classes, Lecture Notes in Math., vol. 131, Springer-Verlag, Berlin and New York, 1970.

23. R. Steinberg, Endomorphisms of linear algebraic groups, Mem. Amer. Math. Soc., no. 80 (1968).

24. Representations of algebraic groups, Nagoya Math. J. 22 (1963), 33-56.

25. Générateurs, relations et revêtements de groupes algébriques, Colloque sur la Théorie des Groupes Algébriques, Bruxelles, 1962.

26. E. Stensholt, An application of Steinberg's twisting groups, Pacific J. Math. 55 (1974), 595-618.

27. F. Timmesfeld, Groups generated by root-involutions. I, II, J. Algebra 33 (1975), 75-134; 35 (1975), $367-441$.

28. A. Wagner, Groups generated by elations, Abh. Math. Sem. Univ. Hamburg 41 (1974), 199-205.

29. M. Aschbacher and G. Seitz, On groups with a standard component of known type, Osaka J. Math. 13 (1976), 439-482.

30. D. Gorenstein, Finite groups, Harper and Row, New York, 1968.

Department of Mathematics, University of Oregon, Eugene, Oregon 97403 